WSRC-TR-2007-00094

Publication Date: June 2007

\title{
Annual Radioactive Waste Tank Inspection Program - 2006
}

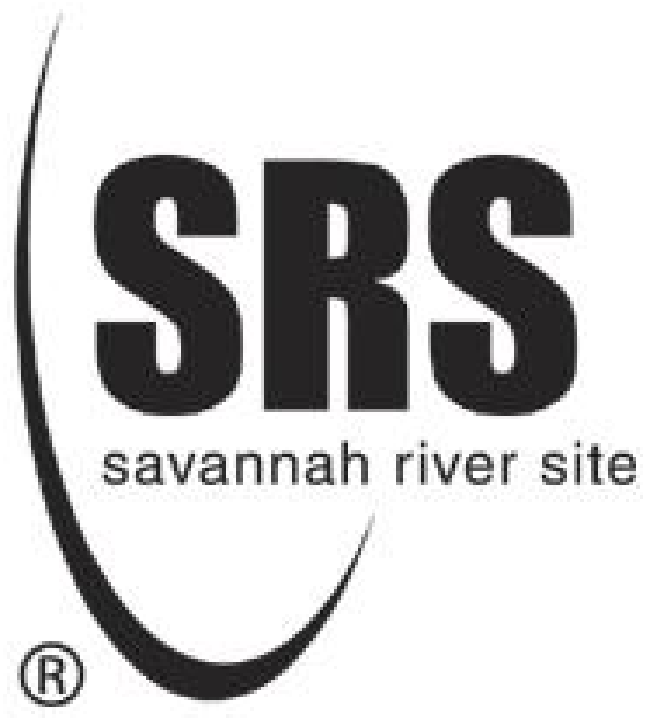

Washington Savannah River Company

Savannah River Site

Aiken, SC 29808

Prepared for the U.S. Department of Energy under Contract No. DE-AC09-96SR18500 


\section{Disclaimer}

This report was prepared as an account of work sponsored by an agency of the United States Government. Neither the United States Government nor any agency thereof, nor any of their employees, makes any warranty, express or implied, or assumes any legal liability or responsibility for the accuracy, completeness, or usefulness of any information, apparatus, product, or process disclosed, or represents that its use would not infringe privately product, process, or service by trade name, trademark, manufacturer, or otherwise does not necessarily constitute or imply its endorsement, recommendation, or favoring by the United States Government or any agency thereof. The views and opinions of authors expressed herein do not necessarily state or reflect those of the United States Government or any agency thereof. 
WSRC-TR-2007-00094

Publication Date: June 2007

\section{Annual Radioactive Waste Tank Inspection Program - 2006}

R. S. Waltz, Jr.

W. R. West

Prepared for the U.S. Department of Energy under Contract No. DE-AC09-96SR18500 
This page intentionally left blank. 


\section{Contents}

Acronyms and Abbreviations .........................................

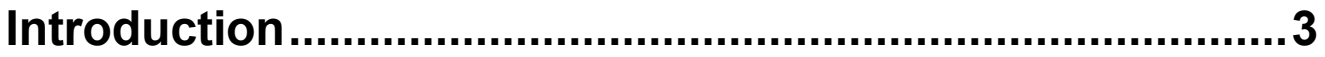

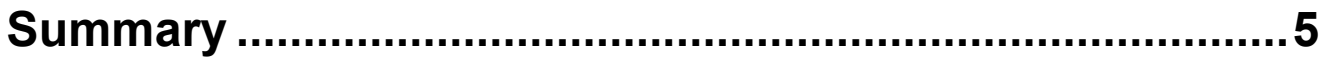

Inspection Program ............................................................ 7

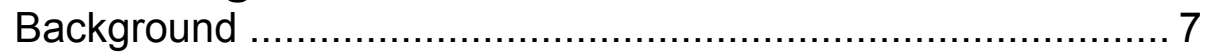

Tank Description .................................................................. 8

Inspection Methods …................................................. 14

Program Implementation .................................................15

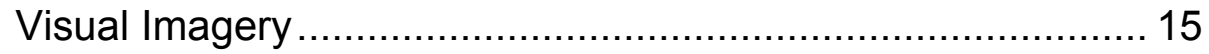

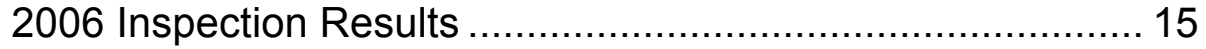

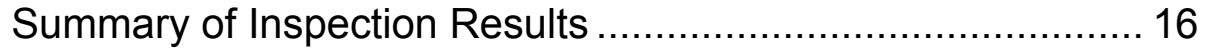

Appendix A-Waste Tanks at SRS ..................................27

Appendix B-Summary of 2006 Inspections .....................29

\section{List of Figures}

1. Type I Waste Storage Tank …….................................. 9

2. Type II Waste Storage Tank ....................................... 10

3. Type IV Waste Storage Tank ........................................ 11

4. Type III Waste Storage Tank ........................................... 12 
This page intentionally left blank. 
WSRC-TR-2007-00094

Annual Waste Tank

Inspection Program - 2006

Acronyms and Abbreviations

\section{Acronyms and Abbreviations}

A

ADMP

ASME

BFV

CCTV

CCWS

COP

CTS

DB

DOE-SR

DP

DSP

DWPF

ERIP

ETF

EVAP

GDL

HLLCP

HPFP

I

IAL

ISI

JB

LDB

LPPP

LPS

MLDB

OD

PP

psig

PT

RCP

SRS

SSD

SSMH

STE

SWS

TTJ

TTP

UT

VB

WAP

WLE

WSRC

WT
Annulus

Advanced Design Mixer Pump

American Society of Mechanical Engineers

Back Flush Valve

Closed Circuit Television

Chromate Cooling Water System

Clean Out Port

Concentrate Transfer System

Diversion Box

Department of Energy-Savannah River

Direct Photography

Digital Still Photography

Defense Waste Processing Facility

Encasement Riser Inspection Port

Effluent Treatment Facility

Evaporator

Gravity Drain Line

High Liquid Level Conductivity Probe

High Point Flush Pit

Interior

Inter-Area Line

In-Service Inspection

Junction Box

Leak Detection Box

Low Point Pump Pit

Leak Probe Sleeve

Modified Leak Detection Box

Outside Diameter

Pump Pit

pounds per square inch gauge

Pump Tank

Reinforced Concrete Pipe

Savannah River Site

Storm Sewer Drain

Storm Sewer Manhole

Shift Technical Engineer

Storm Water Sewer

Telescopic Transfer Jet

Telescopic Transfer Pump

Ultrasonic Nondestructive Examination

Valve Box

Wide-Angle Photography

Waste Line Encasement

Washington Savannah River Company

Waste Transfer Line 
This page intentionally left blank. 
WSRC-TR-2007-00094

Annual Waste Tank

Inspection Program - 2006

Introduction

\section{Introduction}

Aqueous radioactive wastes from Savannah River Site (SRS) separations and vitrification processes are contained in large underground carbon steel tanks. Inspections made during 2006 to evaluate these vessels and other waste handling facilities along with evaluations based on data from previous inspections are the subject of this report. 
This page intentionally left blank. 
WSRC-TR-2007-00094

Annual Waste Tank

Inspection Program - 2006

\section{Summary}

The 2006 inspection program revealed that the structural integrity and waste confinement capability of the Savannah River Site waste tanks were maintained.

A total of 5183 photographs were made and 1482 visual and video inspections were performed during 2006. Additionally, ultrasonic testing was performed on five Waste Tanks (29, 35, 40, 41, and 43) in accordance with approved inspection plans that met the requirements of WSRC-TR2002-00061, Revision 2 "In-Service Inspection Program for High Level Waste Tanks". The Ultrasonic Testing (UT) In-Service Inspections (ISI) are documented in a separate report that is prepared by the ISI programmatic Level III UT Analyst. Tanks 29, 40, 41, and 43 are documented in "Tank Inspection NDE Results for Fiscal Year 2006"; WSRC-TR-2006-00002. Tank 35 will be included in report WSRC-TR-2007-00064 to be published later this year. 
This page intentionally left blank 


\section{Inspection Program}

\section{Background}

Alkaline aqueous radioactive wastes produced at the Savannah River Site are received and managed in large underground tanks. The waste came primarily from nuclear fuel reprocessing operations in the separations areas $(\mathrm{F}$ and $\mathrm{H})$ and contains most of the radioactive fission products from SRS operations. In addition, $\mathrm{H}$ tank farm receives recycle waste from the DWPF vitrification process. Some of this waste has been transferred to F Area waste tanks from $\mathrm{H}$ tank farm. The waste stored in the tanks is present in three phases: sludge, supernate, and salt formed by supernate evaporation and cooling. The supernate and salt phases consist primarily of $\mathrm{NaNO} 3, \mathrm{NaOH}$ and $\mathrm{NaNO} 2$. The fission product content is 1 to 20 curies per gallon (mostly Cs-

$137 / \mathrm{Ba} 137 \mathrm{~m}$ ) for the supernate and up to 5 curies per gallon for the salt. The sludge consists primarily of $\mathrm{MnO} 2, \mathrm{Al}(\mathrm{OH}) 3$ and $\mathrm{Fe}(\mathrm{OH}) 3$ with a fission product content up to 820 curies per gallon (mostly $\mathrm{Sr}-90 / \mathrm{Y}$ 90).

Waste tank leak detection capabilities are essential to meet the primary objective of the SRS radioactive waste management program to manage the waste in such a manner as to minimize the radiation exposure and associated risk to man and his environment over the lifetime of the radionuclides.

The waste tanks are designated compliant or noncompliant based on type of containment, type of leak detection and/or leakage. Noncompliant tanks do not meet current standards for secondary containment and/or leak detection or have leaked. Tanks 1 through 24 are noncompliant. All other waste tanks are compliant.

The detection of leaked waste is based on two principles: disappearance of material from its primary location and appearance of material in a secondary location. At SRS, primary reliance is on the latter because the quantity of the waste detectable in an improper location is much less than that detectable by inventory change in a large tank. Capacity of SRS tanks is 0.75 to 1.3 million gallons. Although rigorous tank inventory surveillance is practiced, primary leak detection methods rely on automatic surveillance of those areas into which the leaked waste is most likely to migrate.

The annulus of each double-wall tank is equipped with at least two single-point conductivity probes for leak detection. These probes are located at the bottom of the annulus and on opposite sides of the tank where possible. The single-wall tanks are built on slabs with a network of leak collection channels that drain to a common sump. Sump levels are continuously monitored with alarm capability in control rooms. Besides the automatic surveillance, routine direct visual surveys are made in the annular spaces, and nonroutine direct visual surveys are made in primary tanks through opened access risers and/or inspection ports in the roof.

In 1961-62, following leakage of waste into the annuli of Tanks 9, 10, 14, and 16, the first remote imaging inspections were made of some tanks using a periscope. Random inspections continued through 1970. A program was initiated in November 1971 to periodically inspect all waste tanks, using remote visual imagery techniques to monitor for corrosion and other degradation, waste leakage, anomalies of any type, and to investigate process or equipment concerns.

Steel thickness measurements have been made periodically of waste tanks using ultrasonic techniques to monitor for general corrosion. An analog-type instrument was 
used in 1967 and 1969 to measure the thickness of the primary wall of selected double-wall tanks. In 1972, a more precise instrument was put in service. About 24,000 measurements made over a period of 14 years (1972 through 1985) indicated that no general thinning trends of SRS tanks had occurred. Steel thickness measurements were resumed in 1994 using an updated ultrasonic testing (UT) system. The system was updated again in 2002 using improved technology to detect thinning, pitting and cracks.

To date, the only visually observed serviceinduced corrosion was in Tank 23, a tank with a unique service history. The upper wall interior surfaces show general corrosion with mild pitting. The pitting is broad but shallow. Evaluation of this condition is documented in DPSP-85-11-4. This tank was used to receive contaminated water from 244-H, the Receiving Basin for Off-Site Fuels, and 245-H, the Resin Regeneration Facility. No increase in the pitting or general corrosion has been observed.

Inspections of waste tanks are complicated by factors such as radiation and radioactive contamination, remote operation as far as 40 feet below grade, and insertion of equipment through small (generally 5 to 8-inchdiameter) access openings. Inspection techniques to circumvent these difficulties have been developed, and they yield quality visual images (photographic) and/or volumetric measurements (UT). The techniques include photographic systems, closed circuit television systems, and ultrasonic systems to measure steel thickness, cracking and pitting.
Waste tank inspection has been important in leak detection. The leaksites in ten of thirteen tanks with documented leaksites have been discovered by direct visual inspection or by one of the remote inspection techniques. Since the inspection program was initiated in 1971, eight tanks were found to have leaksites that were not recognized before the program was implemented. With one exception, Tank 6 in 2001, the annulus conductivity probes in the double-wall tanks were not activated by these leaks because of the small amount of leakage. The leaked waste evaporated to dryness, sealing the leaksites before any leaked waste reached a leak detection probe. However, remote inspections detected the dry deposits of salt in the annuli and/or on the walls of these tanks.

The waste tank Visual Inspection Program and the ISI (UT) programs are ongoing programs. This report gives results of 2006 visual inspections and "limited" UT data and summarizes significant findings of previous UT and visual inspections for each waste tank.

\section{Tank Description}

SRS has subsurface storage tanks of four different designs. All of the tanks are constructed of carbon steel and reinforced concrete. They serve as containment vessels for storage and processing of radioactive wastes. Appendix A lists tank location, design type, project number, and construction period. A brief description of the different tank designs is given in the following paragraphs. 


\section{Type I Tanks}

The 12 original storage tanks constructed between 1951 and 1953 are designated Type I tanks. Tanks 1 through 8 are in F Area and Tanks 9 through 12 are in $\mathrm{H}$ Area. Each primary tank has a capacity of 750,000 gallons. Figure 1 shows the general features of Type I tanks, including the primary tank, the secondary pan, and the concrete support structure. Type I tanks are designated as being noncompliant.

A 9-foot layer of earth was placed over the tanks for radiation shielding. Cooling for each Type I tank is provided by 36 parallel (water pipe) cooling coils.

A dehumidification duct in the annulus of each tank is routed from the tank top to the bottom of the annulus where it encircles all but 8 feet of the tank. The duct has distribution outlets and its cross-sectional area decreases as the distance from the air supply increases. Access to the tank interior is provided at eight locations, and to the annular space at four locations, through riser pipes. Each of the 12 riser pipes is capped at the top with a concrete plug. Each plug is provided with two 5-inchdiameter ports equipped with removable plugs unless equipment has been installed to support waste removal or tank closure activities. The center plug port may provide access through three 4 to 8 inch diameter ports. Some of these ports provide access for inspections.

All welds in the pan and primary tank were radiographically inspected, defects were corrected, and the welds were rechecked radiographically. The welds in the flat bottoms of both the pan and the tank were vacuumtested for leaks. Additionally, both vessels were hydrostatically tested. The water was maintained at full height in the tank for 24 hours before inspection for leaks was made. Cooling water piping was hydrostatically tested at 300 psig and then leak-tested with $100 \mathrm{psig}$ air pressure in the piping.

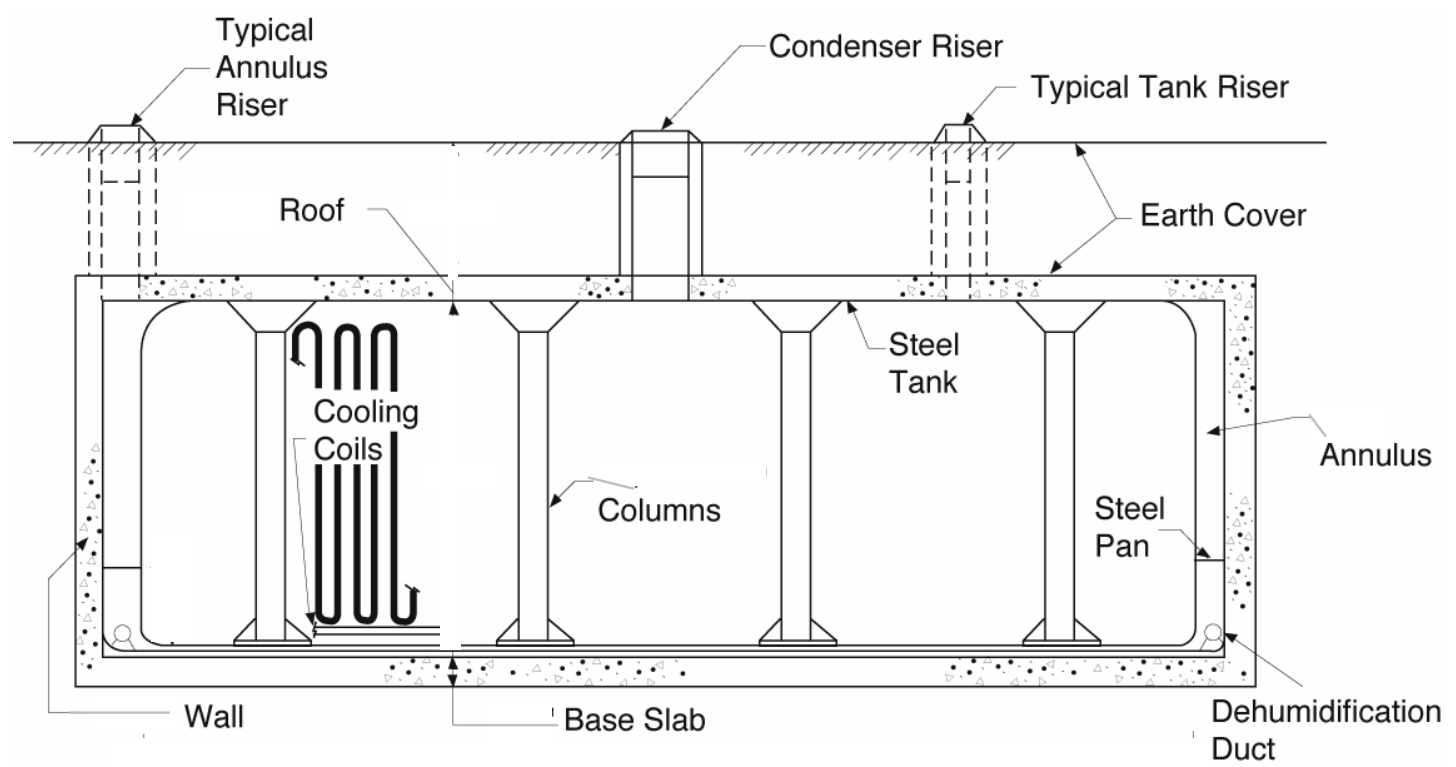

Figure 1. Cooled Waste Storage Tank, Type I (Original 750,000 Gallons). 


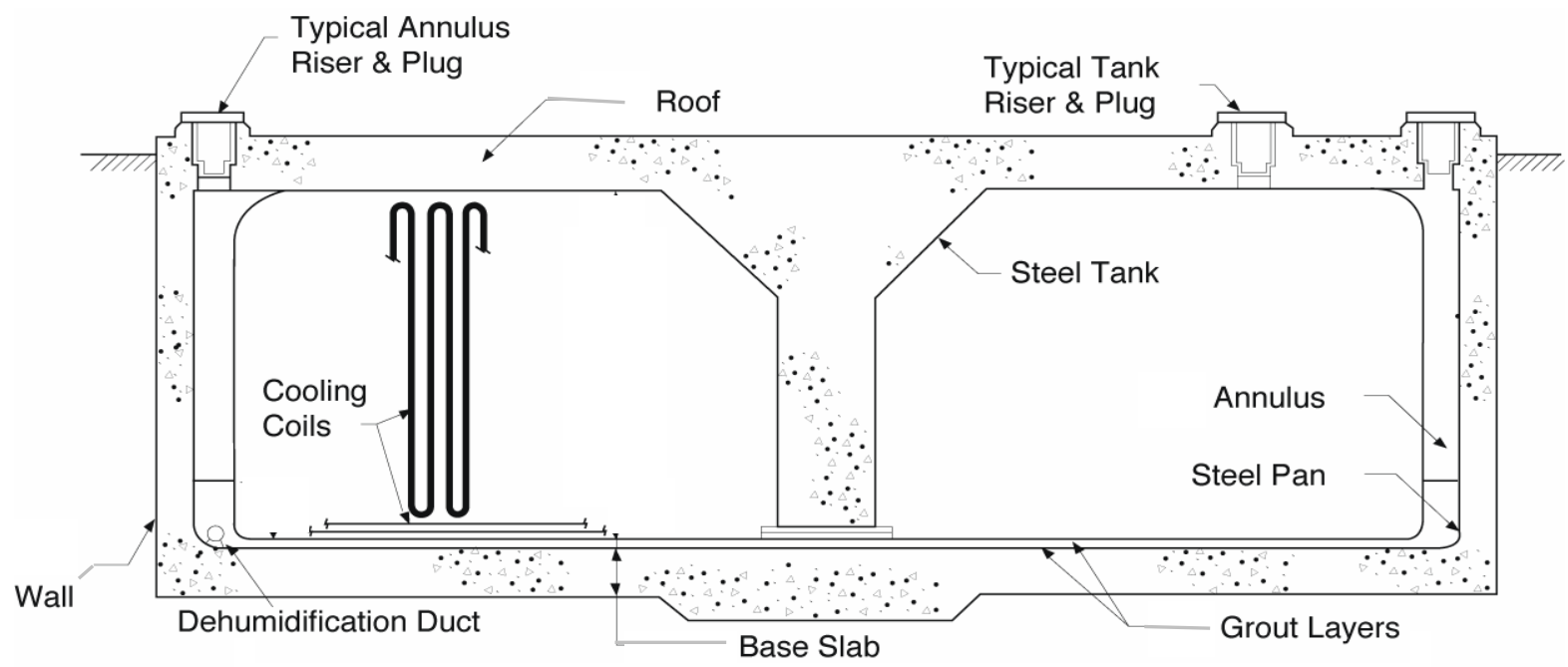

Figure 2. Cooled Waste Storage Tank, Type II (Original 1,030,000 Gallons).

\section{Type II Tanks}

Tanks 13 through 16, constructed in H Area in 1955 and 1956, are designated Type II tanks. Figure 2 is a cross section of this type of tank. Each primary tank has a capacity of 1,030,000 gallons. Type II tanks are designated as being non-compliant.

The primary container for Type II tanks consists of two concentric steel cylinders assembled with a flat bottom and a flat top into a form somewhat like a doughnut. The top and bottom are joined to the outer cylinder by rings of curved knuckle plates. The inner cylinder is flared at the top to accommodate the roof support column. This cylinder is joined to the flat steel top with a continuous butt weld and to a base fastened to the bottom with a continuous T-weld.

The primary tank is set on a 1-inch sand bed within a circular pan of 1/2-inch thick steel plate, 5 feet deep and 5 feet larger in diameter than the primary tank, thus forming an annular space $21 / 2$ feet wide. The tank and pan assembly are surrounded by a cylindrical reinforced concrete enclosure and a flat concrete roof. The tank and pan assembly and the surrounding wall are set on a foundation slab that is 42 inches thick. The roof is supported by both the wall and a central concrete column that fits within the inner cylinder of the vessel. The concrete roof provides radiation shielding; therefore, no earth overburden is required.

Cooling for each Type II tank is provided by 44 parallel (water pipe) cooling coils.

Access to the tank interior is provided at eight locations, and to the annular space at four locations, through riser pipes. Each of the 12 riser pipes is capped at the top with a concrete plug. Each plug is provided with two 5-inch-diameter ports equipped with removable plugs. The ports provide access for inspection. In addition to the four annulus risers, other access openings (10 to 14 additional openings per tank) have been drilled into the annulus of each of these tanks to permit inspection of seventy-three to ninety-six percent of the exterior walls of the primary vessels.

A dehumidification duct in the annulus of each tank is routed from the tank top to the bottom of the annulus where it encircles all but 14 feet of the tank. The duct has distribution outlets, and its cross-sectional area decreases as the distance from the air 
supply increases.

All welds in the primary tanks were radiographically inspected, defects were corrected, and the welds were rechecked radiographically. However, the annulus pans were not inspected radiographically. The welds in the flat bottoms of these pans and the primary tanks were vacuum-tested for leaks, and the primary and secondary vessels were hydrostatically tested. Cooling water piping was hydrostatically tested at $300 \mathrm{psig}$ and then leak-tested, with 100 psig air pressure in the piping.

\section{Type IV Tanks}

Tanks 17 through 24 are single-walluncooled tanks. These tanks were designed for storage of waste that does not require auxiliary cooling. Tanks 17 through 20 were constructed in F Area in 1956 and Tanks 21 through 24 were constructed in $\mathrm{H}$ Area in 1960. Each tank has a capacity of $1,300,000$ gallons. Figure 3 shows the general features of Type IV tanks. Type IV tanks are designated as being non-compliant. Each Type IV tank is basically a steel-lined, prestressed-concrete tank in the form of a vertical cylinder with a domed roof. Carbon steel plates were used to form the cylindrical sides and flat bottom portion of the steel liners. Concrete was built up around the steel vessel by the "shotcrete" technique.

Access to the interior of the tank is provided at six locations through riser pipes. Each riser pipe is capped at the top with a concrete plug. Some of these risers provide access for inspection.

All welds in the steel liners were radiographically inspected. All of the welded tank-bottom seams and the upper seams of the knuckle rings were vacuum leak-tested. Prior to the back-filling operation, each tank was hydrostatically tested by filling with water to the normal fill line. The tank was allowed to remain filled until it was to be placed in use for waste storage.

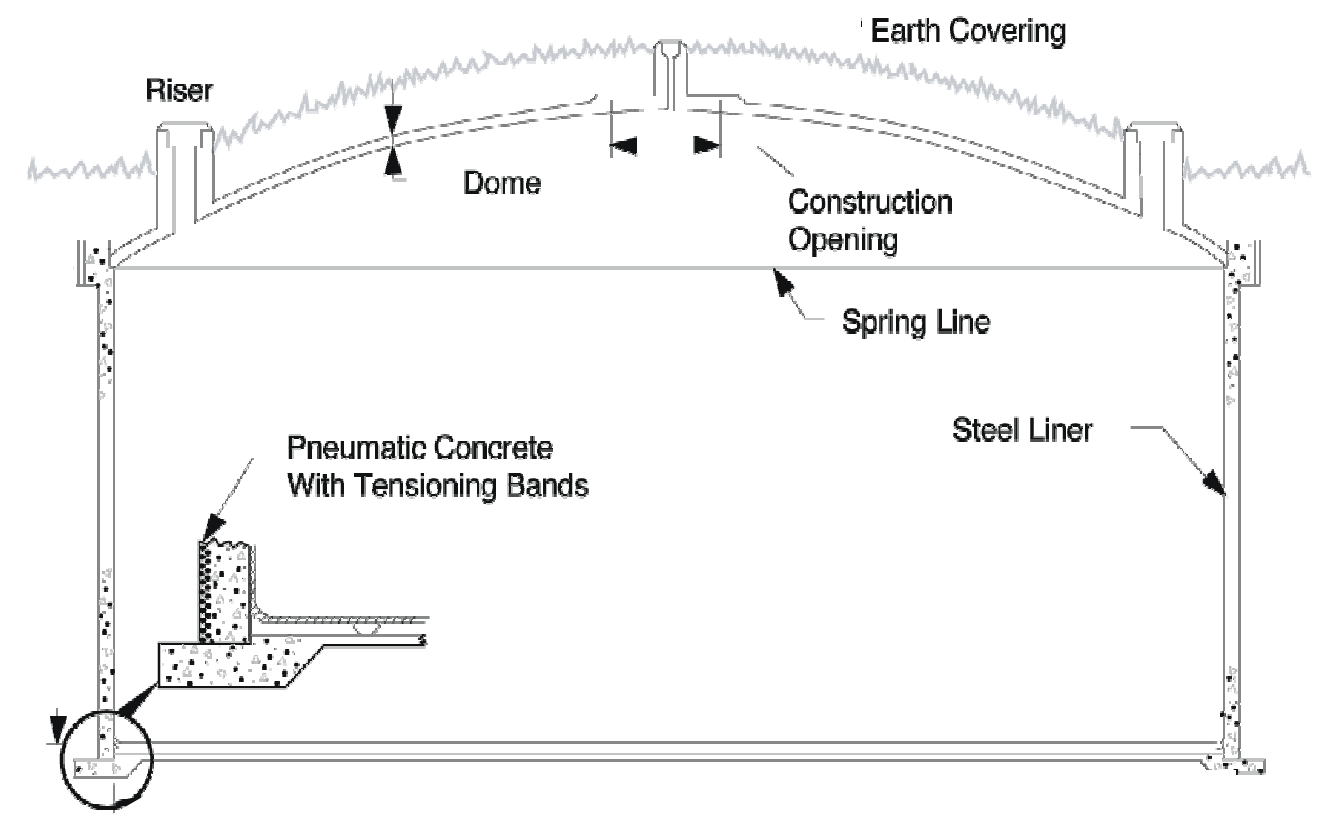

Figure 3. Uncooled Waste Storage Tank, Type IV (Prestressed Concrete Walls, 1,300,000 Gallons). 


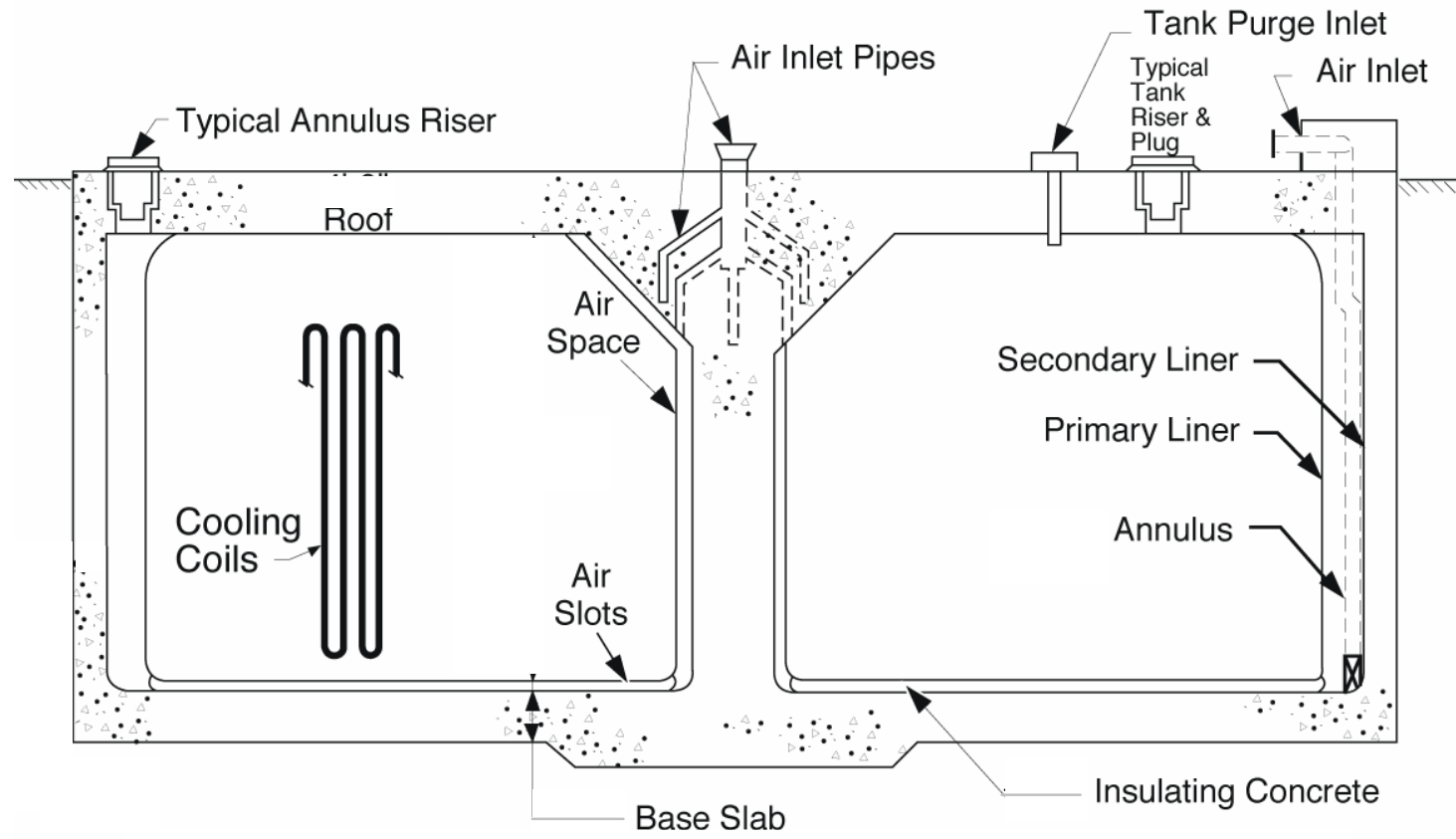

Figure 4. Cooled Waste Storage Tank, Type III (Stress Relieved Primary Liner, 1,300,000 Gallons).

\section{Type III Tanks}

The most recently constructed tanks are designated as Type III tanks (Figure 4).

Twenty-seven tanks were built between 1966 and 1981. Figure 4 shows the general features of the Type III tanks. Type III tanks are designated as compliant. The Type III tank design was developed after an investigation into the causes of the leaks from the primary vessel of the Type I and Type II tanks. The study concluded that the leak-producing mechanism was nitrateinduced, stress-corrosion cracking at sites in or near the weld seams, and that stress relieving after fabrication should eliminate the cracking. For the Type III tanks, means were provided for heating each finished tank to relieve the stresses generated during fabrication. In addition, some stress patterns were avoided, or minimized, by mounting the roof supporting column on the foundation pad rather than on the bottom of the primary tank (as in Types I and II), and by providing an annular clearance around the roof supporting column. Each primary tank holds $1,300,000$ gallons.

Type III tanks are similar to the doughnutlike design of Type II tanks. Each primary vessel is made of two concentric cylinders joined to washer-shaped top and bottom plates by curved knuckle plates.

The primary tank is set on a 6-inch bed of insulating concrete within the secondary containment vessel. The concrete bed is grooved radially so that ventilating air can flow from the inner to the outer annulus. If any waste were to leak from the tank bottom or center annulus wall, liquid would move through the grooves, facilitating detection in the outer annulus.

The secondary vessel is 5 feet larger in diameter than the tank, thus providing an 
outer annulus $21 / 2$ feet wide. Its sidewalls rise to the full height of the primary tank.

The nested two-vessel assembly is surrounded by a cylindrical reinforced concrete wall. The enclosure has a 48-inchthick flat reinforced concrete roof that is supported by the concrete wall and a central column that fits within the inner cylinder of the vessel.

Cooling for the Type III tanks is provided by either deployable (water pipe) cooling coil bundles installed through risers in the tank top, or 23 parallel (water pipe) cooling coils distributed throughout the tank.

A dehumidification duct in the annulus of each tank is routed from the tank top to the bottom of the annulus where it encircles the tank. The duct has distribution outlets and its cross-sectional area decreases as distance from the air supply increases. In these tanks, additional airflow is directed through the inner annulus, passing beneath the primary tank through radial grooves in the concrete base slab, and is exhausted into the outer annulus.

Tanks 29 through 34 were placed in service prior to 1976. These tanks were constructed with annulus riser pipes at four locations providing inspection access through 5 -inchdiameter ports. All other Type III tanks were placed in service after 1976 and have annulus riser pipes at 18 locations that are 8inches in diameter. These ports are equidistant around the tank and provide for inspection of all of the exterior wall of the primary vessel. In 1982, fourteen to sixteen additional 8-inch diameter ports per tank were drilled in the tops of Tanks 29 through 34 to provide adequate access ports for inspection of all of the exterior wall of their primary vessels. All Type III tanks have interior riser pipes at various locations that provide inspection access through ports with diameters ranging from 4 to 8 inches. All inspection access ports are equipped with removable plugs.

All butt welds on the primary tanks were radiographically inspected, except welds on the horizontal roof surface. On the secondary vessels of Tanks 29 through 34, all butt welds joining bottom plates, knuckle plates, and the lowest courses of centercolumn and outer-wall plates, were radiographically inspected. On all other Type III tanks, all plate welds in the secondary tanks were radiographically inspected. All defects were corrected and the welds were rechecked radiographically.

The Quality Assurance Program included inspection of all radiographs by two independent groups of certified weld inspectors, and all radiographs were permanently stored for future reference. All spots on the inside or outside of the primary tanks and the inside of the secondary tanks, where clips or lugs were removed and where other excisions were made, were examined by magnetic particle or liquid penetrant techniques, and any defects were repaired.

All butt welds on the secondary tanks were vacuum leak-tested. All welds in the bottom assemblies of the primary tanks, including knuckle rings and lowest course welds, were vacuum leak-tested before each bottom assembly was lowered into final position, and then tested a second time after the stressrelieving operation. A full hydrostatic test, the filling of each primary tank to a depth of 32 feet and allowing it to stand 48 hours, was conducted after stress relieving. No leaks were found by the hydrostatic tests. All circumferential welds in the pipe loops of the deployable cooling coil bundles below the 1/2-inch-thick plate at the base of the riser plug were radiographed. The assembled cooler piping was tested hydrostatically to 500 psig and halide leaktested at 300 psig. Welds in the distributed cooling coils were radiographed and similarly 
leak-tested.

The primary tank was Post Weld Heat Treated (PWHT) in place after all high temperature work (other than roof attachments) had been completed. PWHT was accomplished in accordance with the general requirements of the ASME Boiler and Pressure Vessel code.

\section{Inspection Methods}

Techniques have been developed for remote examination and evaluation of the waste tanks and waste tank ancillaries. For visual imaging, direct photography systems developed at SRS were the primary method used. Closed circuit television systems were also used where direct photography was not possible or where these systems provided a more comprehensive examination. Only the direct photography systems will be described since the video systems are similar to systems used widely in industry.

Wide-angle direct photography was used for general inspections of double-wall tank annuli and the primary vessels of both double-wall tanks and single-wall tanks. This technique used a camera that surveys a large area in a single photograph. The camera used for wide-angle photography was a Contax G1 camera body, with a Zeiss Hologon $16 \mathrm{~mm} \mathrm{f} / 8$ fixed aperture lens. This lens is distortion free with a field of view of approximately 100 degrees. A bank of four electronic flash units was synchronized with these cameras to provide illumination. This camera is not shielded since residence time in a radiation field is minimal.

Another direct photography technique was used for detailed inspections. The camera is shielded to reduce the degrading effect of ionizing radiation on the photographic film. The camera's residence time in a radiation field for this technique is longer than the wide-angle direct photographic technique (i.e., a few minutes versus a few seconds); hence, shielding is required. The camera used was the Contax G1 camera with a Zeiss Hologon $16 \mathrm{~mm} \mathrm{f} / 8$ lens, the same as used for the wide-angle direct photography.

Illumination is provided by a single electronic flash unit. 


\section{Program Implementation}

\section{Visual Imagery}

The 2006 inspection program used two visual imagery techniques: photography and closed circuit television. The primary inspection methods were direct photography techniques; e.g., making a series of photographs providing detailed views of the tank and wide-angle photography for obtaining overviews of large areas. Closed circuit television systems were generally used to further investigate conditions found during scheduled inspections and to document conditions and troubleshoot process problems in tanks and ancillaries.

The inspection program objective to continuously evaluate the waste tanks was satisfied in 2006 by photographic and videotape documentation. Inspections were made through all accessible annulus risers of the double-wall tanks and at least one inspection was made in the interior of each single-wall tank.

For Tanks 1 through 12, inspections are limited to no more than $25 \%$ of the exterior of the primary vessel wall and the annular space due to limited annulus access. These tanks are continuously monitored for leakage by instrumentation installed in their annuli.

Additionally, for those tanks that have known leaksites in the primary vessel, the supernate phase has been removed, minimized, or the level lowered below the level of known leaksites. Inspections revealing no changes on these tanks are denoted as "Remote visual tank wall inspection revealed no changes since last evaluated."

\section{Inspection Results}

The 2006 inspection program was successfully completed. The annuli of all double-wall tanks were inspected via all accessible risers and the interiors of singlewall tanks remaining in service were inspected. Other inspections of waste tanks and ancillaries were performed as required by operating conditions and equipment performance requirements.

Ultrasonic nondestructive examinations were performed in Tanks 29, 35, 40, 41, and 43. A full scope examination was performed at Tank 29. This included four 8.5 inch wide vertical strips for the entire accessible height, $5 \%$ of a middle horizontal weld, $5 \%$ of the lower knuckle weld, and one vertical weld. The tank was examined for wall thinning, pitting, and crack detection. Reduced augmented scope examinations were performed on Tanks 35, 40, 41, and 43. This consisted of examining one 8.5 inch wide vertical strip for the entire accessible height for wall thinning, pitting and crack detection. Examinations of the secondary vessel wall and annulus floor were performed beneath one riser in both full scope and augmented inspections. Accessible plates on the secondary wall were examined for thinning and pitting. Also, a 10-inch wide strip on the annulus floor was examined for thinning and pitting. No areas of reportable service induced thinning, pitting or cracking were detected on the primary vessel walls, secondary vessel walls, or annulus floor. Several grinding areas in the upper plate of the primary vessel wall in Tank 43 had areas below the $10 \%$ reporting criteria. Several areas of reportable thickness were found in the secondary walls on Tanks 29, 40, and 41. Details of these inspections are documented in WSRC-TR-2006-00002 and WSRC-TR2007-00064. 
WSRC-TR-2007-00094

Annual Waste Tank

Rainwater continued to leak into the annulus of some tanks. Inleakage was evidenced primarily by surface stains, and occasionally by calciferous deposits, changed configuration of salt deposits in the annulus, and mild surface corrosion.

Except as noted above, the conditions of the tanks remained essentially unchanged from the conditions reported in 2005. Details and results for inspections of the tanks and ancillaries performed in 2006 are listed in Appendix B.

\section{Summary of Inspection Results}

The following is a brief description of tank conditions as revealed by inspections and examinations made through 2006. [NOTE: When a tank is "removed from active service", no waste transfers into or out of that tank are allowed.]

\section{Tank 1}

Tank 1 was placed in service in 1954. A small amount of dry waste was observed on the annulus floor in 1969. Subsequent inspections have revealed no additional leakage. Inspection of the exterior wall of the primary vessel is limited to $25 \%$ using existing inspection techniques through the four risers that provide access to the annulus. Visual examinations of the observable portion of the tank wall have not revealed the location of the leak(s). Inspection photographs of the steel surface of the tank and the annulus have shown no significant surface corrosion or other anomalies. Ultrasonic measurements made in 1978, 1979, 1981, 1983, and 1985 showed that no detectable thinning of the tank wall had occurred.

\section{Tank 2}

Tank 2 was placed in service in 1955. Visual examinations of the observable portion $(25 \%)$ of the exterior of the primary vessel wall and the annulus have shown no leakage, significant surface corrosion or other anomalies. Ultrasonic measurements made in $1967,1972,1973,1977,1981$, and 1985 showed no detectable thinning of the tank wall.

\section{Tank 3}

Tank 3 was placed in service in 1956. Visual examinations of the observable portion $(25 \%)$ of the exterior of the primary vessel wall and the annulus have shown no leakage, significant surface corrosion or other anomalies. Ultrasonic measurements made in $1973,1977,1981$, and 1985 showed no detectable thinning of the tank wall.

\section{Tank 4}

Tank 4 was placed in service in 1961. Visual examinations of the observable portion $(25 \%)$ of the exterior of the primary vessel wall and the annulus have shown no leakage, significant surface corrosion or other anomalies. Ultrasonic measurements made in 1973, 1977, 1981, and 1985 showed no detectable thinning of the tank wall.

\section{Tank 5}

Tank 5 was placed in service in 1959. Tank 5 was removed from active service in November 1990. Visual examinations of the observable portions $(25 \%)$ of the exterior of the primary vessel wall and the annulus through calendar year 2000 had shown no leakage, significant surface corrosion, or other anomalies. The tank was returned to active service in 2000 to support tank closure activities. Several months after liquid was added to the tank in 2001, a magnetically mounted wall crawler with a video camera attached was deployed which enabled an additional $50 \%$ of the primary 
vessel wall to be inspected. These inspections revealed 15 leaksites. Less than 5 gallons of waste had reached the annulus floor. Waste was removed from the tank to a level below the lowest known leaksite. During waste removal activities initiated in 2005, three new leaksites were discovered. All leaksites were adjacent to welds and attributed to stress corrosion cracking. Ultrasonic measurements made in 1973, 1977, 1981, and 1985 showed no detectable thinning of the tank wall.

\section{Tank 6}

Tank 6 was placed in service in 1964. Tank 6 was removed from active service in October 1990. Visual examinations of the observable portions $(25 \%)$ of the exterior of the primary vessel wall and the annulus through calendar year 2000 had shown no leakage, significant surface corrosion, or other anomalies. The tank was returned to active service in 2000 to support tank closure activities. The first indication of leakage from the tank was in January 2001 when an annulus conductivity probe alarm was received. Liquid was observed on the annulus floor; however, no leaksites could be located from the four risers used to inspect the tank. In February 2001, a magnetically mounted wall crawler with a video camera attached enabled an additional $48 \%$ of the primary vessel wall to be examined. These inspections revealed 6 leaksites. Approximately 92 gallons of waste reached the annulus floor. Waste was removed from the tank to a level below the lowest known leaksite. All leaksites were adjacent to welds and attributed to stress corrosion cracking. Ultrasonic measurements made in 1974, 1977, 1978, 1979, 1981, and 1985 showed no detectable thinning of the tank wall.

\section{Tank 7}

Tank 7 was placed in service in 1954. Tank 7 was removed from active service in November 1989. Visual examinations of the observable portion (19\%) of the exterior of the primary vessel wall and the annulus have shown no leakage, significant surface corrosion or other anomalies. Ultrasonic measurements made in 1974, 1979, 1981, 1983, and 1985 showed no detectable thinning of the tank wall. Tank 7 was returned to active service in 2002 to support waste removal activities.

\section{Tank 8}

Tank 8 was placed in service in 1956 . Tank 8 was removed from active service in September 1992. Tank 8 was returned to active service in 2001 to support waste removal activities. Visual examinations of the observable portion $(25 \%)$ of the exterior of the primary vessel wall and the annulus have shown no leakage, significant surface corrosion or other anomalies. A magnetically mounted wall crawler with a video camera attached enabled inspection of an additional $34 \%$ of the primary vessel wall and annulus. No leakage, significant surface corrosion, or other anomalies were observed. Ultrasonic measurements made in 1973, 1977, 1981, and 1985 showed no detectable thinning of the tank wall.

\section{Tank 9}

Tank 9 was placed in service in 1955. Liquid waste was observed in the annulus pan in 1957. Currently, the annulus pan contains 8 to 10 inches of salt deposits. Visual examinations of the observable portion $(13 \%)$ of the exterior of the primary vessel wall have shown three leaksites high on the tank wall; 269, 271, and 276 inches above the tank bottom. None of these leaksites are the source of the leaked waste in the annulus pan. The waste leaked at these sites was only enough to form localized 
salt nodules. The leak(s) that are the source of the waste in the annulus pan have not been observed. Inspections have shown no significant surface corrosion, and the ultrasonic measurements made in 1979 and 1983 showed no detectable thinning of the tank wall.

\section{Tank 10}

Tank 10 was placed in service in 1955 . The first indication that Tank 10 had leaked was in 1959 when dry waste was discovered in the annulus pan during a visual inspection. Currently, the annulus pan contains about 2 3 inches of salt deposits. Visual examinations of the observable portion $(19 \%)$ of the exterior of the primary vessel wall have not shown the source of the leaked waste or any other leaksite(s). Inspections have shown no significant surface corrosion, and the ultrasonic measurements made in 1979 and 1983 showed no detectable thinning of the tank wall.

\section{Tank 11}

Tank 11 was placed in service in 1955. Tank 11 was removed from active service in July 1989. Inspections performed in 1974 revealed two leaksites. The leaksites are 189 and 235 inches above the tank bottom. Visual examinations of the observable portions $(25 \%)$ of the exterior of the primary vessel wall have shown no significant surface corrosion, and ultrasonic measurements made in 1973, 1977, 1981, and 1985 showed no detectable thinning of the tank wall. The tank was returned to service in 2004 for waste removal activities.

\section{Tank 12}

Tank 12 was placed in service in 1956. Tank 12 was removed from active service in July 1990. Inspections in 1974 and 1984 revealed two leaksites. The leaksites are 93 and 105 inches above the tank bottom. Inspections in 2004 revealed a new leaksite at 95 inches above the tank bottom. Water additions were made in 2005 to re-wet the dry sludge in preparation of waste removal. Inspections in 2005 revealed two new leaksites at 70 and 129 inches above the tank bottom. Visual examinations of the observable portions $(25 \%)$ of the exterior of the primary vessel wall have shown no significant surface corrosion, and ultrasonic measurements made in 1972, 1973, 1977, 1981, 1983, and 1985 showed no detectable thinning of the tank wall. The tank was returned to service in 2004 for waste removal activities.

\section{Tank 13}

Tank 13 was placed in service in 1956.

Ninety percent of the exterior of the primary vessel wall is observable via the 13 risers that provide access to the annulus. Inspections in 1977 revealed a leaksite 279 inches above the tank bottom. In 1980, another leaksite was discovered 269 inches above the tank bottom. Visual examinations have shown no significant surface corrosion, and ultrasonic measurements made in 1974, 1979, 1985 and 2000 showed no detectable thinning of the tank wall.

\section{Tank 14}

Tank 14 was placed in service in 1957. The first indication that Tank 14 had leaked was in 1959 when dry leaked waste was observed in the annulus pan. Currently, the annulus pan contains 12 to 13 inches of salt deposits. Eighty-nine percent of the exterior of the primary vessel wall is observable via the 18 risers that provide access to the annulus. Inspections have documented 33 leaksites, and it is estimated that there are about 50 leaksites in this tank. All of the documented leaksites are near the bottom circumferential weld that 
is 2.5 feet above the tank bottom, except for one leaksite that was observed approximately 288 inches above the tank bottom. Visual examinations have shown no significant surface corrosion, and ultrasonic measurements made in 1979 and 1983 showed no detectable thinning of the tank wall.

\section{Tank 15}

Tank 15 was placed in service in 1960 . Inspections in 1972 below one of the four risers providing access to the annulus documented two leaksites near the bottom circumferential weld about 2.5 feet above the tank bottom. Twelve additional risers were installed, increasing the observable portion of the primary vessel wall from $25 \%$ to $96 \%$. Inspections in 1973, via the additional risers, revealed eleven other leaksites. Later inspections revealed five other sites where cracks penetrated the steel wall, one was observed in 1994, two were observed in 1997 and two were observed in 2000. Inspections in 2005 revealed an additional leaksite near the bottom circumferential weld. UT Inspections in 2002 revealed a leaksite at 129 inches on the middle circumferential weld. A total of 20 leaksites have been documented. Visual examinations have shown mild corrosion of the steel surfaces in the tank annulus. Ultrasonic measurements made in 1972, 1977, 1980, 1984 and 2002 showed no reportable thinning of the tank wall. Using new equipment capable of detecting cracks and pitting, four partial through-wall crack indications and one complete through-wall crack were documented during UT mapping in 2002 that were previously unknown.

\section{Tank 16}

Tank 16 was placed in service in 1959. Tank 16 was removed from active service in
February 1979. Liquid waste was detected in the annulus pan in 1959. Seventy-three percent of the exterior wall of the primary vessel is observable via the sixteen risers that provide access to the annulus. Inspections in 1961 and 1962, through 13 risers, revealed about 175 leaksites in the tank wall. In October 1961 and March 1962, two 5 3/4inch-diameter samples were cut from the top horizontal circumferential weld of the tank wall about 40 feet apart. Metallurgical examination indicated the cause of the cracks was nitrate-induced stress corrosion. Extensive inspection performed since 1972 indicated that the primary vessel wall has 300 to 350 leaksites. In $1978,70 \%$ of the leaked waste in the annulus pan was removed, leaving an insoluble heel containing approximately 30,000 curies Cs- 137 . Waste removal from the interior of the primary vessel was completed in 1980, and the tank status changed to "out of service". Visual examinations have shown no significant surface corrosion. No ultrasonic steel thickness measurements of the tank were made because of the number of leaksites and the presence of salt nodules on the primary vessel exterior.

\section{Tank 17}

Tank 17 was placed in service in 1961. Visual examinations of the steel liner revealed no evidence of failure, significant surface corrosion or other anomalies. Tank 17 was removed from service and closed December 15, 1997. Inspections have been discontinued.

\section{Tank 18}

Tank 18 was placed in service in 1959. Visual examinations of the steel liner have shown no evidence of failure, significant surface corrosion or other anomalies. Ultrasonic measurements made in 1977, 
1980, and 1983 showed no detectable thinning of the liner bottom. Activities to remove all waste from the tank began in 2001. Tank 18 currently has a waste "heel" of approximately 4300 gallons and is awaiting final closure. The tank was removed from service in 2003.

\section{Tank 19}

Tank 19 was placed in service in 1961 and emptied in 1981. Visual examinations of the steel liner have revealed two failures; i.e. sites where inleakage had occurred. The failures are in the wall of the steel liner at heights of 317 inches and 330 inches. Inspection records photographically document that these leaksites existed before 1994. However, inspections made from the interior of this single-wall (visual inspection of the exterior is not possible) had to track changes in artifacts at the sites by periodic observation to judge that inleakage had occurred. Ultrasonic measurements made in 1982 and 1985 showed no detectable thinning of the liner bottom. Activities to remove all waste from the tank began in 2000. Tank 19 currently has a waste "heel" of approximately 15,100 gallons and is awaiting final closure. The tank was removed from service in 2003.

\section{Tank 20}

Tank 20 was placed in service in 1960. Visual examinations of the steel liner revealed four failure sites. In 1983, leaksites were observed in the wall of the steel liner at heights of 22, 24.5, and 26.5 feet. In 1990, a leaksite was confirmed in the liner wall at a height of 26.25 feet. This site had been suspect since 1984 . This is a single-wall tank with no annulus. The leaksites in the steel liner were detected by inspections made from the tank interior, since inspection of the exterior was not possible. Artifacts observed on the interior wall indicated water had leaked through the steel liner into the tank. Tank 20 was removed from service and closed July 31, 1997. Inspections have been discontinued.

\section{Tank 21}

Tank 21 was placed in service in 1961. Visual examinations of the steel liner have shown no evidence of failure, significant surface corrosion or other anomalies. Ultrasonic measurements made in 1973, 1977, 1980, and 1983 showed no detectable thinning of the liner bottom.

\section{Tank 22}

Tank 22 was placed in service in 1965. Visual examinations of the steel liner have shown no evidence of failure, significant surface corrosion or other anomalies. Water inleakage penetrating the concrete roof was investigated in 1994. This water intrusion was attributed to the presence of perched water on top of the dome (PEC-SMS-960084).

Inspections performed in 2004 showed that the water intrusion through the concrete dome at the NW riser had increased. An Inspection Plan (PDCS-SEG-2005-00032 [7]) was developed for excavation and inspection to assess the structural integrity of the concrete dome, ring girder and to determine the source of the inleakage. In 2006, excavation of the tank overburden and subsequent visual and UT inspections showed the condition of the dome and ring girder to be structurally sound. The source of the inleakage was identified as a cracked 10 " concrete encasement, containing the purge ventilation condensate drain line, allowing perched water to leak through the concrete dome. The area was repaired and a waterproofing material was added.

Subsequent leak checks showed no inleakage of water. The rework of this area eliminated the source of in-leakage into the tank. 
Details of these findings can be found in "Tank -22H Structural Integrity Inspection Report at Northwest Riser" (WSRC-TR2006-00355).

Ultrasonic measurements made in 1974, 1977, 1980, and 1983 showed no detectable thinning of the liner bottom.

\section{Tank 23}

Tank 23 was placed in service in 1964. Visual examinations of the steel liner have revealed corrosion but no evidence of failure. Ultrasonic measurements made in 1973, 1977, 1980, and 1983 showed no detectable thinning of the liner bottom. Examinations of the steel liner have shown rust and tubercles on the surface of the upper portion. This tank served as a receiver tank for inhibited contaminated water from Buildings 244-H, the Receiving Basin for Off-Site Fuels, and 245-H, the Resin Regeneration Facility. This mode of operation exposed only the lower half of the tank to the inhibited contents and exposed the upper half of the tank to a warm humid atmosphere. In 1984, rust and tubercles were cleaned from two small areas, exposing the steel surface. The cleaned liner surface was generally corroded with mild pitting. The pits were broad and shallow. In 1999, cracked or crushed concrete was noted in the tank dome, spanning about fifteen feet immediately above the tank wall. The structural integrity of the dome was evaluated as acceptable per (T-CLC-H00444 and T-CLC-H-00447). The dome meets AC1318-95 code requirements and will continue to be monitored.

\section{Tank 24}

Tank 24 was placed in service in 1963. Visual examinations of the steel liner have shown no evidence of failure, significant surface corrosion or other anomalies.
Ultrasonic measurements made in 1984 showed no detectable thinning of the liner.

\section{Tank 25}

Tank 25 was placed in service in 1980. Visual examinations of $100 \%$ of the exterior of the primary vessel wall and the annulus have shown no leakage, significant surface corrosion or other anomalies. UT measurements made in 1979 and 1983 showed no detectable thinning of the tank wall. UT measurements made in 2004 showed no reportable thinning, pitting or stress corrosion cracking or evidence of service induced tank wall thinning on the primary tank wall. Details of this inspection are documented in WSRC-TR-2004-00166.

\section{Tank 26}

Tank 26 was placed in service in 1980. Visual examinations of $100 \%$ of the exterior of the primary vessel wall and the annulus have shown no leakage, significant surface corrosion or other anomalies. UT measurements made in 1979 and 1983 showed no detectable thinning of the primary tank wall. UT measurements made in 2003 showed no reportable thinning, pitting or stress corrosion cracking or evidence of service induced tank wall thinning on the primary tank wall. Details of this inspection are documented in WSRC-TR-2004-00166.

\section{Tank 27}

Tank 27 was placed in service in 1980.

Visual examinations of $100 \%$ of the exterior of the primary vessel wall and the annulus have shown no leakage, significant surface corrosion or other anomalies. UT measurements made in 1979 and 1983 showed no detectable thinning of the tank wall. UT measurements made in 2005 showed no reportable thinning, pitting or stress corrosion cracking or evidence of 
service induced tank wall thinning on the primary tank wall. Details of this inspection are documented in WSRC-TR-2006-00002.

\section{Tank 28}

Tank 28 was placed in service in 1980. Visual examinations of $100 \%$ of the exterior of the primary vessel wall and the annulus have shown no leakage, significant surface corrosion or other anomalies. UT measurements made in 1979 and 1983 showed no detectable thinning of the tank wall. UT measurements made in 2005 showed no reportable thinning, pitting or stress corrosion cracking in the primary vessel wall. Several small areas of reportable thickness in secondary wall plates 1,2 and 3 were identified. Local thinning was noted at $10 \%$ below nominal thickness in the first secondary wall plate. Details of this inspection are documented in WSRC-TR2005-00039.

\section{Tank 29}

Tank 29 was placed in service in 1971. Visual examinations of $100 \%$ of the exterior of the primary vessel wall and the annulus have shown no leakage, significant surface corrosion or other anomalies. UT measurements made in 1973 and 1974 showed no detectable thinning of the tank wall. UT measurements made in 2006 showed no reportable thinning, pitting, stress corrosion cracking, or evidence of service induced thinning on the primary tank wall. Reportable thickness was detected on one plate of the secondary wall. Reportable thickness was detected on the top plate of the primary tank wall but was attributed to fabrication artifacts. Incipient pitting was detected in the lower plate of the primary tank wall beneath riser P-02. Details of this inspection are documented in WSRC-TR2006-00002.
Tank 30

Tank 30 was placed in service in 1974. Visual examinations of $100 \%$ of the exterior of the primary vessel wall and the annulus have shown no leakage, significant surface corrosion or other anomalies. UT measurements made in 1975 showed no detectable thinning of the tank wall. UT measurements made in 2003 showed no reportable thinning, pitting or stress corrosion cracking or evidence of service induced tank wall thinning on the primary tank wall. Details of this inspection are documented in WSRC-TR-2003-00370.

\section{Tank 31}

Tank 31 was placed in service in 1972.

Visual examinations of $100 \%$ of the exterior of the primary vessel wall and the annulus have shown no leakage, significant surface corrosion or other anomalies. UT measurements made in 2003 showed no reportable thinning, pitting or stress corrosion cracking or evidence of service induced tank wall thinning on the primary tank wall. Details of this inspection are documented in WSRC-TR-2003-00370.

\section{Tank 32}

Tank 32 was placed in service in 1971.

Visual examinations of $100 \%$ of the exterior of the primary vessel wall and the annulus have shown no leakage, significant surface corrosion or other anomalies. UT measurements made in 2003 showed no reportable thinning, pitting or stress corrosion cracking or evidence of service induced tank wall thinning on the primary tank wall. Details of this inspection are documented in WSRC-TR-2003-00370.

\section{Tank 33}

Tank 33 was placed in service in 1969. 
Visual examinations of $100 \%$ of the exterior of the primary vessel wall and the annulus have shown no leakage, significant surface corrosion or other anomalies. UT measurements made in 2005 showed no reportable thinning, pitting or stress corrosion cracking or evidence of service induced tank wall thinning on the primary tank wall. Details of this inspection are documented in WSRC-TR-2006-00002.

\section{Tank 34}

Tank 34 was placed in service in 1972. Visual examinations of $100 \%$ of the exterior of the primary vessel wall and the annulus have shown no leakage, significant surface corrosion or other anomalies. UT measurements made in 2003 showed no reportable thinning, pitting or stress corrosion cracking or evidence of service induced tank wall thinning on the primary tank wall. Details of this inspection are documented in WSRC-TR-2003-00370.

\section{Tank 35}

Tank 35 was placed in service in 1977. Visual examinations of $100 \%$ of the exterior of the primary vessel wall and the annulus have shown no leakage, significant surface corrosion or other anomalies. UT measurements made in 1977, 1981, and 1985 showed no detectable thinning of the tank wall. UT measurements made in 2006 showed no reportable thinning, pitting, stress corrosion cracking, or evidence of service induced thinning on the primary tank wall. Reportable thicknesses were detected in the top plate of the primary vessel, but are attributed to fabrication artifacts. Reportable thicknesses were detected in one of four secondary wall plates as well as the annulus floor. Details of this inspection are documented in WSRC-TR-2007-00064.

\section{Tank 36}

Tank 36 was placed in service in 1977. Visual examinations of $100 \%$ of the exterior of the primary vessel wall and the annulus have shown no leakage, significant surface corrosion or other anomalies. UT measurements made in 1977, 1981, and 1985 showed no detectable thinning of the tank wall.

\section{Tank 37}

Tank 37 was placed in service in 1978.

Visual examinations of $100 \%$ of the exterior of the primary vessel wall and the annulus have shown no leakage, significant surface corrosion or other anomalies. UT measurements made in 1977, 1981, and 1985 showed no detectable thinning of the tank wall.

\section{Tank 38}

Tank 38 was placed in service in 1981. Visual examinations of $100 \%$ of the exterior of the primary vessel wall and the annulus have shown no leakage, significant surface corrosion or other anomalies. UT measurements made in 1980, 1981, and 1984 showed no detectable thinning of the tank wall.

\section{Tank 39}

Tank 39 was placed in service in 1982.

Visual examinations of $100 \%$ of the exterior of the primary vessel wall and the annulus have shown no leakage, significant surface corrosion or other anomalies. UT measurements made in 1980, 1981, 1984, and 1985 showed no detectable thinning of the tank wall. UT measurements made in 2005 showed no reportable thinning, pitting or stress corrosion cracking or evidence of service induced tank wall thinning on the primary tank wall. Several grinding areas in 
the upper plate of the primary vessel wall had areas below the $10 \%$ reporting criteria. Several small areas of reportable thickness were detected in secondary wall plates 1,2 and 4. Details of this inspection are documented in WSRC-TR-2006-00002.

\section{Tank 40}

Tank 40 was placed in service in 1986. Visual examinations of $100 \%$ of the exterior of the primary vessel wall and the annulus have shown no leakage, significant surface corrosion or other anomalies. UT measurements were made in 1980, 1981, and 1984. Thickness mapping was performed in 1996 using the P-scan System to provide reference measurements for the future. UT measurements made in 2006 showed no reportable pitting, stress corrosion cracking, or evidence of service induced thinning on the primary tank wall. Reportable thicknesses were detected in the top plate of the primary tank wall but were attributed to fabrication artifacts. Reportable thicknesses were detected in 2 of 4 secondary wall plates. Reportable thicknesses were detected on the annulus floor, but were attributed to pre-service pitting. Details of this inspection are documented in WSRC-TR-2006-00002.

\section{Tank 41}

Tank 41 was placed in service in 1982. Visual examinations of $100 \%$ of the exterior of the primary vessel wall and the annulus have shown no leakage, significant surface corrosion or other anomalies. UT measurements made in 1980, 1981, and 1984 showed no detectable thinning of the tank wall. UT measurements made in 2006 showed no reportable pitting, stress corrosion cracking, or evidence of service induced thinning on the primary tank wall. Reportable thicknesses were detected in the top knuckle and plate of the primary tank and in one plate on the secondary wall.
Reportable thicknesses were detected on the annulus floor but were attributed to preservice pitting. Details of this inspection are documented in WSRC-TR-2006-00002.

\section{Tank 42}

Tank 42 was placed in service in 1982. Visual examinations of $100 \%$ of the exterior of the primary vessel wall and the annulus have shown no leakage, significant surface corrosion or other anomalies. Ultrasonic thickness measurements were made in 1980, 1981, 1984, 1985 and 1990. Thickness mapping was performed in 1995 and 1996 using the P-scan System to provide reference measurements for the future. UT measurements made in 2004 showed no reportable thinning, pitting or stress corrosion cracking or evidence of service induced tank wall thinning on the primary tank wall. A few small areas of reportable thickness were detected on the secondary tank wall with the minimum thickness being 0.338 inches on plate one, 0.336 inches on plate two, and 0.332 inches on plate three. Details of this inspection are documented in WSRC-TR-2005-00039.

\section{Tank 43}

Tank 43 was placed in service in 1982. Visual examinations of $100 \%$ of the exterior of the primary vessel wall and the annulus have shown no leakage, significant surface corrosion or other anomalies. UT measurements made in 1980, 1981, 1984, and 1985 showed no detectable thinning of the tank wall. UT measurements made in 2006 showed no reportable thinning, pitting, stress corrosion cracking, or evidence of service induced thinning on the primary tank wall. Reportable thicknesses were detected in the top and middle plates of the primary vessel, but are attributed to fabrication artifacts. Reportable thicknesses were detected in two of four secondary wall plates 
as well as the annulus floor. Grinding areas were observed in all plates. Details of this inspection are documented in WSRC-TR2006-00002.

\section{Tank 44}

Tank 44 was placed in service in 1982. Visual examinations of $100 \%$ of the exterior of the primary vessel wall and the annulus have shown no leakage, significant surface corrosion or other anomalies. UT measurements made in 1980, 1981, and 1984 showed no detectable thinning of the tank wall. UT measurements made in 2005 showed no reportable thinning, pitting or stress corrosion cracking or evidence of service induced tank wall thinning on the primary tank wall. Several small areas of reportable thickness in secondary wall plates 1, 2 and 4 were detected. Details of this inspection are documented in WSRC-TR2005-00039.

\section{Tank 45}

Tank 45 was placed in service in 1982. Visual examinations of $100 \%$ of the exterior of the primary vessel wall and the annulus have shown no leakage, significant surface corrosion or other anomalies. UT measurements made in 1980, 1981, and 1984 showed no detectable thinning of the tank wall. UT measurements made in 2005 showed no reportable thinning, pitting or stress corrosion cracking or evidence of service induced tank wall thinning on the primary tank wall. One small area of reportable thickness was detected in the second wall plate on the secondary liner. Details of this inspection are documented in WSRC-TR-2005-00039.

\section{Tank 46}

Tank 46 was placed in service as an emergency spare tank in 1980. It was placed in waste storage service in 1994 when it began receiving concentrate from the $2 \mathrm{~F}$ evaporator. Visual examinations of $100 \%$ of the exterior of the primary vessel wall and the annulus have shown no significant surface corrosion or other anomalies. UT measurements made in 1980, 1981, and 1984 showed no detectable thinning of the tank wall. UT measurements made in 2005 showed no reportable thinning, pitting or stress corrosion cracking or evidence of service induced tank wall thinning on the primary tank wall. One small area of reportable thickness was detected in the first wall plate on the secondary liner. Details of this inspection are documented in WSRCTR-2005-00039.

\section{Tank 47}

Tank 47 was placed in service in 1980 . Visual examinations of $100 \%$ of the exterior of the primary vessel wall and the annulus have shown no leakage, significant surface corrosion or other anomalies. UT measurements made in 1980, 1981, and 1984 showed no detectable thinning of the tank wall. UT measurements made in 2005 showed no reportable thinning, pitting or stress corrosion cracking or evidence of service induced tank wall thinning on the primary tank wall. Two small areas of reportable thickness were detected in the fourth wall plate on the secondary liner. An incipient pit was noted in the lower plate of the primary vessel wall. Details of this inspection are documented in WSRC-TR2005-00039.

\section{Tank 48}

Tank 48 was placed in service in 1983. Visual examinations of $100 \%$ of the exterior of the primary vessel wall and the annulus have shown no leakage, significant surface corrosion or other anomalies. UT measurements were made in 1982 prior to 
placing the tank in service. Thickness mapping was performed in 1994, 1995, 1996, and 1997 using the P-scan System to provide reference measurements for the future. UT measurements made in 2004 showed no reportable thinning, pitting or stress corrosion cracking or evidence of service induced tank wall thinning on the primary tank wall. Details of this inspection are documented in WSRC-TR-2004-00166.

\section{Tank 49}

Tank 49 was placed in service in 1983. Visual examinations of $100 \%$ of the exterior of the primary vessel wall and the annulus have shown no leakage, significant surface corrosion or other anomalies. UT measurements were made in 1982 prior to placing the tank in service. Thickness mapping was performed in 1995 using the Pscan System to provide reference measurements for the future. UT measurements made in 2004 showed no reportable thinning, pitting or stress corrosion cracking or evidence of service induced tank wall thinning on the primary tank wall. Details of this inspection are documented in WSRC-TR-2005-00039.

\section{Tank 50}

Tank 50 was placed in service in 1983. Visual examinations of $100 \%$ of the exterior of the primary vessel wall and the annulus have shown no leakage, significant surface corrosion or other anomalies. UT measurements were made in 1982 prior to placing the tank in service. Thickness mapping was performed in 1994 and 1995 using the P-scan System to provide reference measurements for the future. UT measurements made in 2004 showed no reportable thinning, pitting or stress corrosion cracking or evidence of service induced tank wall thinning on the primary tank wall. Details of this inspection are documented in WSRC-TR-2004-00166.

\section{Tank 51}

Tank 51 was placed in service in 1986.

Visual examinations of $100 \%$ of the exterior of the primary vessel wall and the annulus have shown no leakage, significant surface corrosion or other anomalies. UT measurements were made in 1982 prior to placing the tank in service. Thickness mapping was performed in 1996 and 1997 using the P-scan System to provide reference measurements for the future. UT measurements made in 2004 showed no reportable thinning, pitting or stress corrosion cracking or evidence of service induced tank wall thinning on the primary tank wall. A few small areas of reportable thickness were detected on the secondary tank wall with the minimum thickness being 0.332 inches on plate one and 0.336 inches on plate two. Details of this inspection are documented in WSRC-TR-2005-00039. 


\section{Appendix A-Waste Tanks at SRS}

\section{SRS Waste Tank Specifications}

\begin{tabular}{|c|c|c|c|c|}
\hline$\underline{\text { Number }}$ & Type & $\begin{array}{l}\text { Project } \\
\text { Number }\end{array}$ & $\begin{array}{c}\text { Construction } \\
\text { Period }\end{array}$ & $\begin{array}{c}\text { Type of* } \\
\text { Construction }\end{array}$ \\
\hline $1-8$ & I & 8980 & $1951-1953$ & $\begin{array}{l}\text { Double wall- } \\
\text { cooled }\end{array}$ \\
\hline $9-12$ & I & 8980 & $1951-1953$ & $\begin{array}{l}\text { Double wall- } \\
\text { cooled }\end{array}$ \\
\hline $13-16$ & II & $\begin{array}{c}8980 \\
\text { P. W. O }\end{array}$ & $1955-1956$ & $\begin{array}{l}\text { Double wall- } \\
\text { cooled }\end{array}$ \\
\hline $17-20$ & IV & 981030 & 1956 & $\begin{array}{l}\text { Single wall- } \\
\text { uncooled }\end{array}$ \\
\hline $21-24$ & IV & 981089 & 1960 & $\begin{array}{l}\text { Single wall- } \\
\text { uncooled }\end{array}$ \\
\hline $25-28$ & IIIA & $\begin{array}{l}9 \mathrm{~S} 1493 \\
(75-1-\mathrm{a})\end{array}$ & $1975-1978$ & $\begin{array}{l}\text { Double wall- } \\
\text { cooled }\end{array}$ \\
\hline $29-32$ & III & 981232 & $1966-1970$ & $\begin{array}{l}\text { Double wall- } \\
\text { cooled }\end{array}$ \\
\hline $33-34$ & III & 9S0974 & $1969-1972$ & $\begin{array}{l}\text { Double wall- } \\
\text { cooled }\end{array}$ \\
\hline $35-37$ & IIIA & $\begin{array}{l}9 \mathrm{~S} 1463 \\
(74-1-\mathrm{a})\end{array}$ & $1974-1977$ & $\begin{array}{l}\text { Double wall- } \\
\text { cooled }\end{array}$ \\
\hline $38-43$ & IIIA & $\begin{array}{l}\text { 9S1618 } \\
\text { (76-8-A) }\end{array}$ & $1976-1980$ & $\begin{array}{l}\text { Double wall- } \\
\text { cooled }\end{array}$ \\
\hline $44-47$ & IIIA & $9 S 1747$ & $1977-1980$ & $\begin{array}{l}\text { Double wall- } \\
\text { cooled }\end{array}$ \\
\hline $48-51$ & IIIA & $\begin{array}{l}9 \mathrm{~S} 1828 \\
(78-18-\mathrm{b})\end{array}$ & $1978-1981$ & $\begin{array}{l}\text { Double wall- } \\
\text { cooled }\end{array}$ \\
\hline
\end{tabular}

* Tanks 32 and 35 have removable, roof-supported cooling coils. Tanks 30, 33, and 34 have bottom-supported deployable cooling coils. Tanks 29 and 31 have some deployable and some close-packed cooling assemblies, all bottom supported. All other cooled tanks have permanently installed cooling coils, roof-supported in Type I and II and bottom-supported in Type III tanks. 
This page intentionally left blank 


\begin{tabular}{|c|c|c|c|c|c|c|c|c|}
\hline \multirow{2}{*}{$\frac{\text { AREA }}{F}$} & \multirow{2}{*}{$\begin{array}{c}\begin{array}{c}\text { TANK OR } \\
\text { ANCILLARY }\end{array} \\
01\end{array}$} & \multicolumn{2}{|c|}{$\frac{\text { ACCESS OPENING }}{(A \text { OR I) }}$} & \multirow{2}{*}{$\frac{\text { DATE }}{09 / 29 / 06}$} & \multicolumn{3}{|c|}{$\begin{array}{l}\text { INSPECTION METHOD } \\
\text { IDENTIFICATION NUMBER }\end{array}$} & \multirow{2}{*}{$\begin{array}{l}\text { REMARKS } \\
\text { Remote visual tank wall inspection } \\
\text { revealed no changes since last } \\
\text { evaluated on } 8 / 23 / 05 \text {. }\end{array}$} \\
\hline & & East & (A) & & DP & I & P06275:01-27 & \\
\hline $\mathrm{F}$ & 01 & East & (A) & 09/29/06 & DP & l & P06275:25-26 & $\begin{array}{l}\text { The magnetically mounted tank wall } \\
\text { thermocouple was properly } \\
\text { positioned per T-DS-G-00010. }\end{array}$ \\
\hline $\mathrm{F}$ & 01 & East & (A) & $10 / 06 / 06$ & CCTV & I & 1370 & $\begin{array}{l}\text { The conductivity probe was properly } \\
\text { deployed per J-JX-G-0001. }\end{array}$ \\
\hline $\mathrm{F}$ & 01 & North & (A) & 09/29/06 & DP & l & P06274:01-25 & $\begin{array}{l}\text { Remote visual tank wall inspection } \\
\text { revealed no changes since last } \\
\text { evaluated on } 6 / 23 / 05 \text {. The annulus } \\
\text { jet showed no degradation per T-DS- } \\
\text { G- } 00043 \text {. }\end{array}$ \\
\hline $\mathrm{F}$ & 01 & North & (A) & 09/29/06 & DP & l & P06276:01-28 & $\begin{array}{l}\text { Remote visual inspection of the } \\
\text { secondary vessel wall revealed no } \\
\text { areas of concern since last evaluated } \\
\text { on } 4 / 5 / 04 \text {. }\end{array}$ \\
\hline $\mathrm{F}$ & 01 & South & (A) & 09/29/06 & DP & l & P06273:01-27 & $\begin{array}{l}\text { Remote visual tank wall inspection } \\
\text { revealed no changes since last } \\
\text { evaluated on } 8 / 23 / 05 \text {. }\end{array}$ \\
\hline $\mathrm{F}$ & 01 & West & (A) & 09/29/06 & DP & l & P06272:01-22 & $\begin{array}{l}\text { Remote visual tank wall inspection } \\
\text { revealed no changes since last } \\
\text { evaluated on } 8 / 23 / 05 \text {. }\end{array}$ \\
\hline $\mathrm{F}$ & 01 & West & (A) & $10 / 06 / 06$ & CCTV & l & 1370 & $\begin{array}{l}\text { The conductivity probe was properly } \\
\text { deployed per J-JX-G-0001. }\end{array}$ \\
\hline $\mathrm{F}$ & 02 & East & (A) & 09/08/06 & DP & l & P06268:01-22 & $\begin{array}{l}\text { Remote visual inspection of the } \\
\text { secondary vessel wall revealed no } \\
\text { areas of concern since last evaluated } \\
\text { on } 7 / 6 / 05 \text {. }\end{array}$ \\
\hline $\mathrm{F}$ & 02 & East & (A) & 09/08/06 & DP & I & P06267:01-22 & $\begin{array}{l}\text { Remote visual tank wall inspection } \\
\text { revealed no areas of concern since } \\
\text { last evaluated on } 7 / 6 / 05 \text {. }\end{array}$ \\
\hline $\mathrm{F}$ & 02 & East & $(\mathrm{A})$ & 09/08/06 & DP & l & P06267:19 & $\begin{array}{l}\text { The magnetically mounted tank wall } \\
\text { thermocouple was properly } \\
\text { positioned per T-DS-G-00010. }\end{array}$ \\
\hline $\mathrm{F}$ & 02 & North & (A) & 08/28/06 & WAP & l & P06254:03 & $\begin{array}{l}\text { Remote visual tank wall inspection } \\
\text { revealed no areas of concern since } \\
\text { last evaluated on } 7 / 06 / 05 \text {. The } \\
\text { annulus jet showed no degradation } \\
\text { per T-DS-G-00043. }\end{array}$ \\
\hline $\mathrm{F}$ & 02 & North & (A) & $10 / 06 / 06$ & CCTV & l & 1370 & $\begin{array}{l}\text { The conductivity probe was properly } \\
\text { deployed per J-JX-G-0001. }\end{array}$ \\
\hline
\end{tabular}




\begin{tabular}{|c|c|c|c|c|c|c|c|c|}
\hline \multirow{2}{*}{$\frac{\text { AREA }}{F}$} & \multirow{2}{*}{$\begin{array}{c}\text { TANK OR } \\
\text { ANCILLARY } \\
02\end{array}$} & \multicolumn{2}{|c|}{$\frac{\text { ACCESS OPENING }}{(\text { (A OR I) }}$} & \multirow{2}{*}{$\begin{array}{l}\text { DATE } \\
08 / 28 / 06\end{array}$} & \multicolumn{3}{|c|}{$\begin{array}{l}\text { INSPECTION METHOD } \\
\text { IDENTIFICATION NUMBER }\end{array}$} & \multirow{2}{*}{$\begin{array}{l}\text { REMARKS } \\
\text { Remote visual tank wall inspection } \\
\text { revealed no areas of concern since } \\
\text { last evaluated on } 7 / 06 / 05 \text {. }\end{array}$} \\
\hline & & South & (A) & & WAP & l & P06254:01 & \\
\hline $\mathrm{F}$ & 02 & South & (A) & $10 / 06 / 06$ & CCTV & l & 1370 & $\begin{array}{l}\text { The conductivity probe was properly } \\
\text { deployed per J-JX-G-0001. }\end{array}$ \\
\hline $\mathrm{F}$ & 02 & West & (A) & $08 / 28 / 06$ & WAP & I & P06254:02 & $\begin{array}{l}\text { Remote visual tank wall inspection } \\
\text { revealed no areas of concern since } \\
\text { last evaluated on } 7 / 06 / 05 \text {. }\end{array}$ \\
\hline $\mathrm{F}$ & 03 & East & (A) & 08/28/06 & WAP & I & P06255:02 & $\begin{array}{l}\text { Remote visual tank wall inspection } \\
\text { revealed no areas of concern since } \\
\text { last evaluated on } 7 / 11 / 05 \text {. }\end{array}$ \\
\hline $\mathrm{F}$ & 03 & North & (A) & 08/28/06 & WAP & l & P06255:01 & $\begin{array}{l}\text { Remote visual tank wall inspection } \\
\text { revealed no areas of concern since } \\
\text { last evaluated on } 7 / 11 / 05 \text {. The } \\
\text { annulus jet showed no degradation } \\
\text { per T-DS-G-00043. }\end{array}$ \\
\hline $\mathrm{F}$ & 03 & North & (A) & 10/06/06 & CCTV & l & 1370 & $\begin{array}{l}\text { The conductivity probe was properly } \\
\text { deployed per J-JX-G-0001. }\end{array}$ \\
\hline $\mathrm{F}$ & 03 & South & (A) & $08 / 28 / 06$ & WAP & l & P06255:03 & $\begin{array}{l}\text { Remote visual tank wall inspection } \\
\text { revealed no areas of concern since } \\
\text { last evaluated on } 7 / 11 / 05 \text {. }\end{array}$ \\
\hline $\mathrm{F}$ & 03 & South & (A) & 10/06/06 & CCTV & l & 1370 & $\begin{array}{l}\text { The conductivity probe was properly } \\
\text { deployed per J-JX-G-0001. }\end{array}$ \\
\hline $\mathrm{F}$ & 03 & West & (A) & 09/08/06 & DP & l & P06266:01-22 & $\begin{array}{l}\text { Remote visual inspection of the } \\
\text { secondary vessel wall revealed no } \\
\text { areas of concern since last evaluated } \\
\text { on } 4 / 2 / 04 \text {. }\end{array}$ \\
\hline $\mathrm{F}$ & 03 & West & (A) & 09/08/06 & DP & I & P06265:01-22 & $\begin{array}{l}\text { Remote visual tank wall inspection } \\
\text { revealed no areas of concern since } \\
\text { last evaluated on } 7 / 11 / 05 \text {. }\end{array}$ \\
\hline $\mathrm{F}$ & 03 & West & (A) & 09/08/06 & DP & l & P06265:19 & $\begin{array}{l}\text { The magnetically mounted tank wall } \\
\text { thermocouple was properly } \\
\text { positioned per T-DS-G-00010. }\end{array}$ \\
\hline $\mathrm{F}$ & 04 & East & (A) & 09/08/06 & DP & l & P06259:01-22 & $\begin{array}{l}\text { Remote visual tank wall inspection } \\
\text { revealed no areas of concern since } \\
\text { last evaluated on } 8 / 13 / 05 \text {. }\end{array}$ \\
\hline $\mathrm{F}$ & 04 & East & (A) & 09/08/06 & DP & & $06259: 13,14,19$ & $\begin{array}{l}\text { The magnetically mounted tank wall } \\
\text { thermocouple was properly } \\
\text { positioned per T-DS-G-00010. }\end{array}$ \\
\hline $\mathrm{F}$ & 04 & East & $(\mathrm{A})$ & 09/08/06 & DP & l & P06260:01-22 & $\begin{array}{l}\text { Remote visual inspection of the } \\
\text { secondary vessel wall revealed no } \\
\text { areas of concern since last evaluated } \\
\text { on } 4 / 6 / 04 \text {. }\end{array}$ \\
\hline
\end{tabular}




\begin{tabular}{|c|c|c|c|c|c|c|c|c|}
\hline \multirow{2}{*}{ AREA } & \multirow{2}{*}{$\begin{array}{c}\text { TANK OR } \\
\text { ANCILLARY } \\
04\end{array}$} & \multicolumn{2}{|c|}{$\begin{array}{c}\text { ACCESS OPENING } \\
\text { (A OR I) }\end{array}$} & \multirow{2}{*}{$\frac{\text { DATE }}{06 / 29 / 06}$} & \multicolumn{3}{|c|}{$\begin{array}{l}\text { INSPECTION METHOD } \\
\text { IDENTIFICATION NUMBER }\end{array}$} & \multirow{2}{*}{$\begin{array}{l}\qquad \text { REMARKS } \\
\text { Remote visual tank wall inspection } \\
\text { revealed no areas of concern since } \\
\text { last evaluated on } 8 / 14 / 05 \text {. }\end{array}$} \\
\hline & & North & (A) & & WAP & l & P06182:01 & \\
\hline $\mathrm{F}$ & 04 & North & (A) & $10 / 06 / 06$ & CCTV & I & 1370 & $\begin{array}{l}\text { The conductivity probe was properly } \\
\text { deployed per J-JX-G-0001. }\end{array}$ \\
\hline $\mathrm{F}$ & 04 & South & (A) & 06/29/06 & WAP & I & P06182:03 & $\begin{array}{l}\text { Remote visual tank wall inspection } \\
\text { revealed no areas of concern since } \\
\text { last evaluated on } 8 / 14 / 05 \text {. The } \\
\text { annulus jet showed no degradation } \\
\text { per T-DS-G-00043. }\end{array}$ \\
\hline $\mathrm{F}$ & 04 & South & (A) & 10/06/06 & CCTV & l & 1370 & $\begin{array}{l}\text { The conductivity probe was properly } \\
\text { deployed per J-JX-G-0001. }\end{array}$ \\
\hline $\mathrm{F}$ & 04 & West & (A) & 06/29/06 & WAP & l & P06182:02 & $\begin{array}{l}\text { Remote visual tank wall inspection } \\
\text { revealed no areas of concern since } \\
\text { last evaluated on } 8 / 14 / 05 \text {. }\end{array}$ \\
\hline $\mathrm{F}$ & 05 & East & (A) & 09/30/06 & WAP & I & P06173:02 & $\begin{array}{l}\text { Remote visual tank wall inspection } \\
\text { revealed no changes since last } \\
\text { evaluated on } 7 / 12 / 05 \text {. }\end{array}$ \\
\hline $\mathrm{F}$ & 05 & North & (A) & $04 / 12 / 06$ & CCTV & l & 1343 & $\begin{array}{l}\text { The conductivity probe was properly } \\
\text { positioned per J-JX-G-0001. }\end{array}$ \\
\hline $\mathrm{F}$ & 05 & North & (A) & 09/30/06 & WAP & I & P06173:01 & $\begin{array}{l}\text { Remote visual tank wall inspection } \\
\text { revealed no changes since last } \\
\text { evaluated on } 7 / 12 / 05 \text {. The annulus } \\
\text { jet showed no degradation per T-DS- } \\
\text { G-00043. }\end{array}$ \\
\hline $\mathrm{F}$ & 05 & South & (A) & 03/30/06 & CCTV & I & 1343 & $\begin{array}{l}\text { The conductivity probe was properly } \\
\text { positioned per J-JX-G-0001. }\end{array}$ \\
\hline $\mathrm{F}$ & 05 & South & (A) & 09/08/06 & DP & I & P06262:01-22 & $\begin{array}{l}\text { Remote visual tank wall inspection } \\
\text { revealed no changes since last } \\
\text { evaluated on } 7 / 12 / 05 \text {. Salt nodules } \\
\text { had formed on the previously } \\
\text { identified leaksites reactivated during } \\
\text { waste removal activities. Salt } \\
\text { deposits had also cascaded down } \\
\text { the tank wall from the leaksites. }\end{array}$ \\
\hline $\mathrm{F}$ & 05 & South & (A) & 09/08/06 & DP & l & P06263:01-22 & $\begin{array}{l}\text { Remote visual inspection of the } \\
\text { secondary vessel wall revealed no } \\
\text { areas of concern since last evaluated } \\
\text { on } 4 / 2 / 04 \text {. }\end{array}$ \\
\hline $\mathrm{F}$ & 05 & West & (A) & $01 / 08 / 06$ & CCTV & l & 1337 & $\begin{array}{l}\text { CCTV was used to determine the } \\
\text { elevation of a leaksite discovered in } \\
2005 \text { during waste removal activities. } \\
\text { The leaksite is } 35 \text { inches above the } \\
\text { tank bottom. }\end{array}$ \\
\hline
\end{tabular}




\begin{tabular}{|c|c|c|c|c|c|c|c|c|}
\hline \multirow{2}{*}{ AREA } & \multirow{2}{*}{$\begin{array}{c}\begin{array}{c}\text { TANK OR } \\
\text { ANCILLARY }\end{array} \\
05\end{array}$} & \multicolumn{2}{|c|}{$\frac{\text { ACCESS OPENING }}{\text { (A OR I) }}$} & \multirow{2}{*}{$\frac{\text { DATE }}{\text { 04/20/06 }}$} & \multicolumn{3}{|c|}{$\begin{array}{l}\text { INSPECTION METHOD } \\
\text { IDENTIFICATION NUMBER }\end{array}$} & \multirow{2}{*}{$\begin{array}{l}\text { REMARKS } \\
\text { The magnetically mounted tank wall } \\
\text { thermocouple was properly } \\
\text { positioned per T-DS-G-00010. }\end{array}$} \\
\hline & & West & (A) & & CCTV & l & 1370 & \\
\hline $\mathrm{F}$ & 05 & West & (A) & 09/08/06 & DP & l & P06264:01-22 & $\begin{array}{l}\text { Remote visual tank wall inspection } \\
\text { revealed no changes since last } \\
\text { evaluated on } 7 / 12 / 05 \text {. Salt nodules } \\
\text { had formed on the previously } \\
\text { identified leaksites reactivated during } \\
\text { waste removal activities. Salt } \\
\text { deposits had also cascaded down } \\
\text { the tank wall from the leaksites. }\end{array}$ \\
\hline $\mathrm{F}$ & 05 & West & (A) & 09/08/06 & DP & I & P06264:19 & $\begin{array}{l}\text { The magnetically mounted tank wall } \\
\text { thermocouple was properly } \\
\text { positioned per T-DS-G-00010. }\end{array}$ \\
\hline $\mathrm{F}$ & 05 & 02 & (I) & 01/19/06 & CCTV & l & 1336 & $\begin{array}{l}\text { CCTV was used to document } \\
\text { conditions and position of the slurry } \\
\text { mixer pump installed in riser } 3 \text {. } \\
\text { Several roof mounted cooling coil } \\
\text { hangers near the riser had become } \\
\text { detached from the roof and one } \\
\text { cooling coil had broken in half at the } \\
\text { top loop. }\end{array}$ \\
\hline $\mathrm{F}$ & 05 & 02 & (I) & 03/19/06 & WAP & I & P06035:01-08 & $\begin{array}{l}\text { Inspection documented waste } \\
\text { configuration in the tank after waste } \\
\text { removal. }\end{array}$ \\
\hline $\mathrm{F}$ & 05 & 07 & (I) & 03/19/06 & WAP & I & P06034:01-06 & $\begin{array}{l}\text { Inspection documented waste } \\
\text { configuration in the tank after waste } \\
\text { removal. }\end{array}$ \\
\hline $\mathrm{F}$ & 05 & Center & (I) & $02 / 09 / 06$ & WAP & l & P06012:01-23 & $\begin{array}{l}\text { Inspection documented conditions in } \\
\text { the tank after liquid was transferred } \\
\text { to Tank } 7 \text {. }\end{array}$ \\
\hline $\mathrm{F}$ & 06 & East & (A) & 09/30/06 & WAP & I & P06282:02 & $\begin{array}{l}\text { Remote visual tank wall inspection } \\
\text { revealed no changes since last } \\
\text { evaluated on } 8 / 23 / 05 \text {. }\end{array}$ \\
\hline$F$ & 06 & East & (A) & $11 / 03 / 06$ & CCTV & I & 1499 & $\begin{array}{l}\text { The magnetically mounted tank wall } \\
\text { thermocouple was properly } \\
\text { positioned per T-DS-G-00010. }\end{array}$ \\
\hline $\mathrm{F}$ & 06 & North & (A) & 09/30/06 & WAP & I & P06282:01 & $\begin{array}{l}\text { Remote visual tank wall inspection } \\
\text { revealed no changes since last } \\
\text { evaluated on } 8 / 23 / 05 \text {. }\end{array}$ \\
\hline $\mathrm{F}$ & 06 & South & (A) & 09/30/06 & DP & l & P06280:01-22 & $\begin{array}{l}\text { Remote visual tank wall inspection } \\
\text { revealed no changes since last } \\
\text { evaluated on } 8 / 23 / 05 \text {. The annulus } \\
\text { jet showed no degradation per T-DS- } \\
\text { G- } 00043 \text {. }\end{array}$ \\
\hline
\end{tabular}




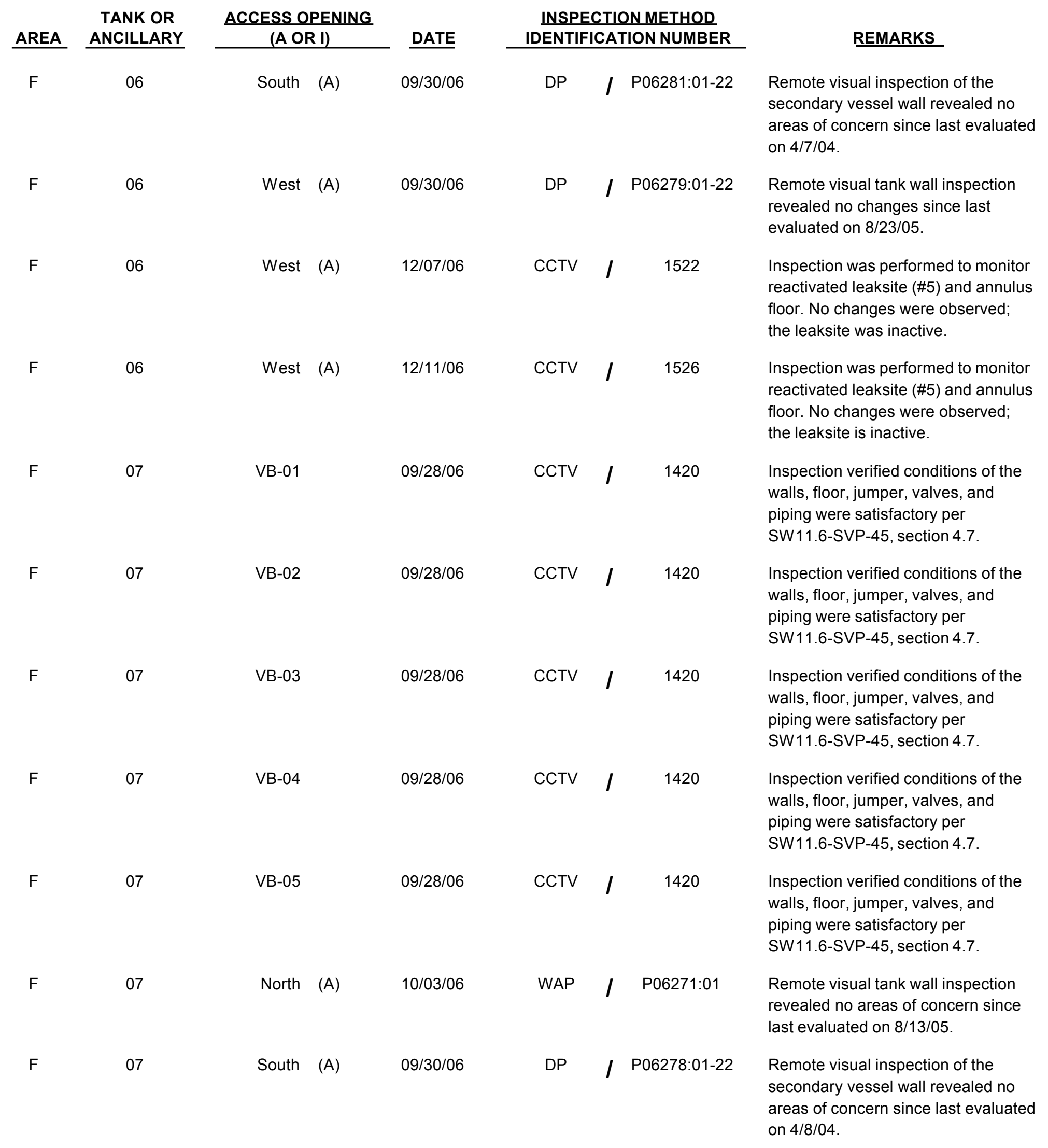




\begin{tabular}{|c|c|c|c|c|c|c|c|c|}
\hline \multirow{2}{*}{$\frac{\text { AREA }}{F}$} & \multirow{2}{*}{$\begin{array}{c}\text { TANK OR } \\
\text { ANCILLARY } \\
07\end{array}$} & \multicolumn{2}{|c|}{$\frac{\text { ACCESS OPENING }}{\text { (A OR I) }}$} & \multirow{2}{*}{$\begin{array}{c}\text { DATE } \\
09 / 30 / 06\end{array}$} & \multicolumn{3}{|c|}{$\begin{array}{l}\text { INSPECTION METHOD } \\
\text { IDENTIFICATION NUMBER }\end{array}$} & \multirow{2}{*}{$\begin{array}{l}\text { REMARKS } \\
\text { Remote visual tank wall inspection } \\
\text { revealed no areas of concern since } \\
\text { last evaluated on } 8 / 13 / 05 \text {. The } \\
\text { annulus jet showed no degradation } \\
\text { per T-DS-G-00043. }\end{array}$} \\
\hline & & South & (A) & & DP & l & P06277:01-22 & \\
\hline $\mathrm{F}$ & 07 & West & (A) & 07/10/06 & CCTV & I & 1420 & $\begin{array}{l}\text { The magnetically mounted tank wall } \\
\text { thermocouple was properly } \\
\text { positioned per T-DS-G-00010. }\end{array}$ \\
\hline $\mathrm{F}$ & 07 & West & (A) & $10 / 03 / 06$ & WAP & l & P06271:02 & $\begin{array}{l}\text { Remote visual tank wall inspection } \\
\text { revealed no areas of concern since } \\
\text { last evaluated on } 8 / 13 / 05 \text {. }\end{array}$ \\
\hline $\mathrm{F}$ & 07 & Center & $(\mathrm{I})$ & 05/16/06 & CCTV & I & 1365 & $\begin{array}{l}\text { Inspection verified the proper } \\
\text { deployment of the HLLCP. }\end{array}$ \\
\hline $\mathrm{F}$ & 08 & LBD-17 & & $12 / 20 / 06$ & CCTV & l & 1120 & $\begin{array}{l}\text { Inspection verified conditions of the } \\
\text { walls, floor, valves, and piping were } \\
\text { satisfactory per SW11.6-SVP-45, } \\
\text { section } 4.7 \text {. The conductivity probes } \\
\text { were properly positioned. }\end{array}$ \\
\hline $\mathrm{F}$ & 08 & East & (A) & 09/04/06 & WAP & l & P06256:02 & $\begin{array}{l}\text { Remote visual tank wall inspection } \\
\text { revealed no areas of concern since } \\
\text { last evaluated on } 8 / 13 / 05 \text {. }\end{array}$ \\
\hline $\mathrm{F}$ & 08 & East & (A) & $10 / 07 / 06$ & CCTV & l & 1370 & $\begin{array}{l}\text { The magnetically mounted tank wall } \\
\text { thermocouple was properly } \\
\text { positioned per T-DS-G-00010. }\end{array}$ \\
\hline $\mathrm{F}$ & 08 & North & (A) & 09/04/06 & WAP & I & P06256:03 & $\begin{array}{l}\text { Remote visual tank wall inspection } \\
\text { revealed no areas of concern since } \\
\text { last evaluated on } 8 / 13 / 05 \text {. The } \\
\text { annulus jet showed no degradation } \\
\text { per T-DS-G-00043. }\end{array}$ \\
\hline $\mathrm{F}$ & 08 & South & (A) & 09/04/06 & WAP & I & P06256:01 & $\begin{array}{l}\text { Remote visual tank wall inspection } \\
\text { revealed no areas of concern since } \\
\text { last evaluated on } 8 / 13 / 05 \text {. }\end{array}$ \\
\hline $\mathrm{F}$ & 08 & West & (A) & 09/08/06 & DP & I & P06258:01-22 & $\begin{array}{l}\text { Remote visual inspection of the } \\
\text { secondary vessel wall revealed no } \\
\text { areas of concern since last evaluated } \\
\text { on } 4 / 2 / 04 \text {. }\end{array}$ \\
\hline $\mathrm{F}$ & 08 & West & (A) & 09/08/06 & DP & I & P06257:01-22 & $\begin{array}{l}\text { Remote visual tank wall inspection } \\
\text { revealed no areas of concern since } \\
\text { last evaluated on } 8 / 13 / 05 \text {. }\end{array}$ \\
\hline $\mathrm{H}$ & 09 & South & (A) & 03/08/06 & WAP & l & P06023:01 & $\begin{array}{l}\text { Remote visual tank wall inspection } \\
\text { revealed no changes since last } \\
\text { evaluated on } 12 / 4 / 05 \text {. }\end{array}$ \\
\hline
\end{tabular}




\begin{tabular}{|c|c|c|c|c|c|c|c|c|}
\hline \multirow{2}{*}{$\begin{array}{l}\text { AREA } \\
\mathrm{H}\end{array}$} & \multirow{2}{*}{$\begin{array}{c}\text { TANK OR } \\
\text { ANCILLARY } \\
09\end{array}$} & \multicolumn{2}{|c|}{$\frac{\text { ACCESS OPENING }}{(A \text { OR I) }}$} & \multirow{2}{*}{$\begin{array}{c}\text { DATE } \\
10 / 30 / 06\end{array}$} & \multicolumn{3}{|c|}{$\begin{array}{l}\text { INSPECTION METHOD } \\
\text { IDENTIFICATION NUMBER }\end{array}$} & \multirow{2}{*}{$\begin{array}{l}\text { REMARKS } \\
\text { The conductivity probe was properly } \\
\text { deployed per J-JX-G-0001. }\end{array}$} \\
\hline & & South & (A) & & CCTV & l & 1370 & \\
\hline $\mathrm{H}$ & 09 & West & (A) & $03 / 08 / 06$ & $\mathrm{DP}$ & l & P06024:01-23 & $\begin{array}{l}\text { Remote visual tank wall inspection } \\
\text { revealed no changes since last } \\
\text { evaluated on } 12 / 4 / 05 \text {. }\end{array}$ \\
\hline $\mathrm{H}$ & 09 & West & (A) & 03/08/06 & $\mathrm{DP}$ & l & P06024:14 & $\begin{array}{l}\text { The magnetically mounted tank wall } \\
\text { thermocouple was properly } \\
\text { positioned per T-DS-G-00010. }\end{array}$ \\
\hline $\mathrm{H}$ & 09 & West & (A) & 03/08/06 & $\mathrm{DP}$ & I & P06024:18 & $\begin{array}{l}\text { The conductivity probe was properly } \\
\text { deployed per J-JX-G-0001. }\end{array}$ \\
\hline $\mathrm{H}$ & 09 & West & (A) & 03/08/06 & $\mathrm{DP}$ & l & P06025:01-22 & $\begin{array}{l}\text { Remote visual inspection of the } \\
\text { secondary vessel wall revealed no } \\
\text { changes since last evaluated on } \\
4 / 23 / 04 \text {. }\end{array}$ \\
\hline $\mathrm{H}$ & 10 & East & (A) & 03/09/06 & WAP & l & P06026:02 & $\begin{array}{l}\text { Remote visual tank wall inspection } \\
\text { revealed no changes since last } \\
\text { evaluated on } 5 / 10 / 05 \text {. }\end{array}$ \\
\hline $\mathrm{H}$ & 10 & East & (A) & 07/19/06 & CCTV & l & 1429 & $\begin{array}{l}\text { The conductivity probe was properly } \\
\text { deployed per J-JX-G-0001. }\end{array}$ \\
\hline $\mathrm{H}$ & 10 & East & (A) & $10 / 10 / 06$ & CCTV & l & 1370 & $\begin{array}{l}\text { The conductivity probe was properly } \\
\text { deployed per J-JX-G-0001. }\end{array}$ \\
\hline $\mathrm{H}$ & 10 & East & (A) & 10/10/06 & CCTV & l & 1370 & $\begin{array}{l}\text { The magnetically mounted tank wall } \\
\text { thermocouple was properly } \\
\text { positioned per T-DS-G-00010. }\end{array}$ \\
\hline $\mathrm{H}$ & 10 & North & (A) & 03/09/06 & WAP & l & P06026:01 & $\begin{array}{l}\text { Remote visual tank wall inspection } \\
\text { revealed no changes since last } \\
\text { evaluated on } 9 / 21 / 05 \text {. }\end{array}$ \\
\hline $\mathrm{H}$ & 10 & North & (A) & $10 / 30 / 06$ & CCTV & l & 1370 & $\begin{array}{l}\text { The conductivity probe was properly } \\
\text { deployed per J-JX-G-0001. }\end{array}$ \\
\hline $\mathrm{H}$ & 10 & West & (A) & 03/09/06 & $\mathrm{DP}$ & I & P06027:01-25 & $\begin{array}{l}\text { Remote visual tank wall inspection } \\
\text { revealed no changes since last } \\
\text { evaluated on } 5 / 11 / 05 \text {. }\end{array}$ \\
\hline $\mathrm{H}$ & 10 & West & (A) & 03/09/06 & $\mathrm{DP}$ & l & P06028:01-21 & $\begin{array}{l}\text { Remote visual inspection of the } \\
\text { secondary vessel wall revealed no } \\
\text { areas of concern since last evaluated } \\
\text { on } 4 / 25 / 04 \text {. }\end{array}$ \\
\hline $\mathrm{H}$ & 11 & East & (A) & 03/07/06 & DP & l & P06033:01-26 & $\begin{array}{l}\text { Remote visual tank wall inspection } \\
\text { revealed no changes since last } \\
\text { evaluated on } 5 / 2 / 05 \text {. }\end{array}$ \\
\hline
\end{tabular}




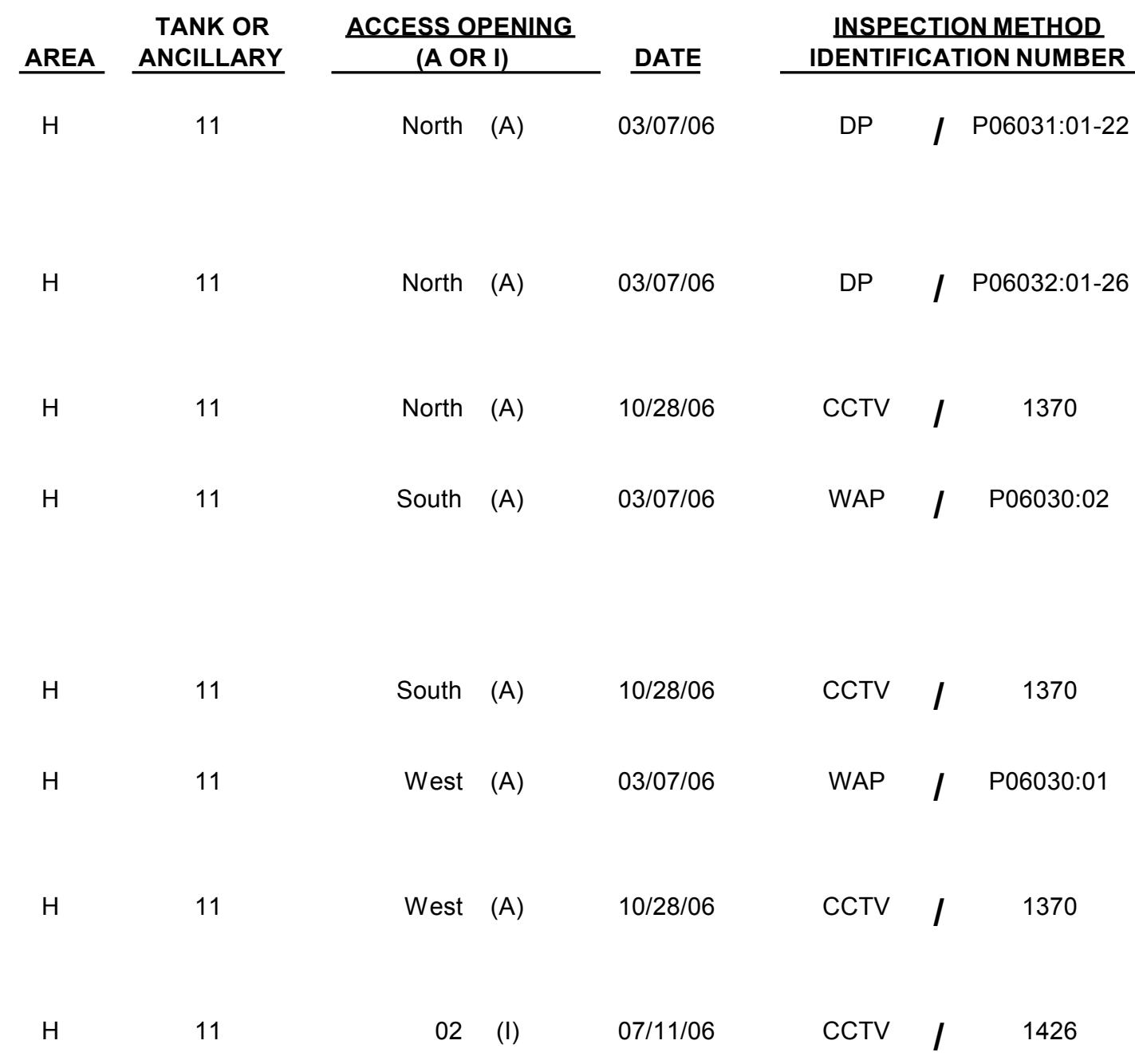

\section{REMARKS}

Remote visual inspection of the secondary vessel wall revealed no areas of concern since last evaluated on $5 / 1 / 04$.

Remote visual tank wall inspection revealed no changes since last evaluated on $5 / 2 / 05$.

The conductivity probe was properly deployed per J-JX-G-0001.

Remote visual tank wall inspection revealed no changes since last evaluated on $11 / 17 / 05$. The annulus jet showed no degradation per T-DSG-00043.

The conductivity probe was properly deployed per J-JX-G-0001.

Remote visual tank wall inspection revealed no changes since last evaluated on 5/2/05.

The magnetically mounted tank wall thermocouple was properly positioned per T-DS-G-00010.

Inspection was performed to document the condition of the tank wall and cooling coils and look for any evidence of general corrosion. Some mild surface corrosion was observed on the lower loop of the cooling coils in some areas however the tank wall appears to be in very good condition. The evaluation is documented in LWO-LWE-2006 -00029 .

Inspection was performed to document the condition of the tank wall and cooling coils and look for any evidence of general corrosion. Some mild surface corrosion was observed on the lower loop of the cooling coils in some areas however the tank wall appears to be in very good condition. The evaluation is documented in LWO-LWE-2006 -00029 . 


\begin{tabular}{|c|c|c|c|c|c|c|}
\hline \multirow{2}{*}{$\frac{\text { AREA }}{\mathrm{H}}$} & \multirow{2}{*}{$\begin{array}{c}\begin{array}{c}\text { TANK OR } \\
\text { ANCILLARY }\end{array} \\
11\end{array}$} & $\frac{\text { ACCESS OPENING }}{\text { (A OR I) }}$ & \multirow{2}{*}{$\begin{array}{l}\text { DATE } \\
07 / 11 / 06\end{array}$} & \multicolumn{3}{|c|}{$\begin{array}{l}\text { INSPECTION METHOD } \\
\text { IDENTIFICATION NUMBER }\end{array}$} \\
\hline & & $05 \quad$ (I) & & CCTV & I & 1426 \\
\hline $\mathrm{H}$ & 12 & East (A) & $01 / 03 / 06$ & WAP & I & P06001:02 \\
\hline $\mathrm{H}$ & 12 & East (A) & $10 / 15 / 06$ & CCTV & I & 1370 \\
\hline $\mathrm{H}$ & 12 & North (A) & $01 / 03 / 06$ & $\mathrm{DP}$ & I & P06002:01-28 \\
\hline
\end{tabular}

$\mathrm{H}$

12

$\mathrm{H}$

$\mathrm{H}$

$\mathrm{H}$

$\mathrm{H}$

$\mathrm{H}$
12

12

12

12

12
North (A)

01/03/06

North (A)

South (A)

South

(A)

West

(A)

02 (I)

$08 / 24 / 06$
01/03/06

$11 / 01 / 06$

01/03/06

$11 / 01 / 06$

DP

| P06003:01-27

Inspection was performed to document the condition of the tank wall and cooling coils and look for any evidence of general corrosion. Some mild surface corrosion was observed on the lower loop of the cooling coils in some areas however the tank wall appears to be in very good condition. The evaluation is documented in LWO-LWE-2006 -00029 .

Remote visual tank wall inspection revealed no changes since last evaluated on 10/4/05.

The magnetically mounted tank wall thermocouple was properly positioned per T-DS-G-00010.

Remote visual tank wall inspection revealed no changes since last evaluated on $12 / 3 / 05$. The annulus jet showed no degradation per T-DSG-00043.

Remote visual inspection of the secondary vessel wall revealed no areas of concern since last evaluated on $4 / 25 / 05$.

The conductivity probe was properly deployed per J-JX-G-0001.

Remote visual tank wall inspection revealed no changes since last evaluated on 10/4/05.

The conductivity probe was properly deployed per J-JX-G-0001.

Remote visual tank wall inspection revealed no changes since last evaluated on 10/4/05.

Inspection identified five broken cooling coils. One coil was broken in two places for a total of six locations where the coils have failed. The coils are broken at weld seams or where the coils were tack welded to the roof mounted hangers. Several coil hangers were also broken away from either the coil or roof. 


\begin{tabular}{|c|c|c|c|c|c|c|c|c|}
\hline \multirow{2}{*}{ AREA } & \multirow{2}{*}{$\begin{array}{c}\begin{array}{c}\text { TANK OR } \\
\text { ANCILLARY }\end{array} \\
13\end{array}$} & \multicolumn{2}{|c|}{$\frac{\text { ACCESS OPENING }}{(A \text { OR I) }}$} & \multirow{2}{*}{$\begin{array}{c}\text { DATE } \\
\text { 05/18/06 }\end{array}$} & \multicolumn{3}{|c|}{$\begin{array}{l}\text { INSPECTION METHOD } \\
\text { IDENTIFICATION NUMBER }\end{array}$} & \multirow{2}{*}{$\begin{array}{l}\quad \text { REMARKS } \\
\text { Remote visual inspection of the } \\
\text { secondary vessel wall revealed no } \\
\text { areas of concern since last evaluated } \\
\text { on } 9 / 12 / 05 \text {. }\end{array}$} \\
\hline & & 010 & (A) & & DP & I & P06092:01-24 & \\
\hline $\mathrm{H}$ & 13 & 010 & (A) & $05 / 18 / 06$ & DP & l & P06093:01-26 & $\begin{array}{l}\text { Remote visual tank wall inspection } \\
\text { revealed no changes since last } \\
\text { evaluated on } 9 / 12 / 05 \text {. }\end{array}$ \\
\hline $\mathrm{H}$ & 13 & 032 & (A) & $05 / 18 / 06$ & DP & I & P06094:01-28 & $\begin{array}{l}\text { Remote visual tank wall inspection } \\
\text { revealed no changes since last } \\
\text { evaluated on } 5 / 28 / 05 \text {. }\end{array}$ \\
\hline $\mathrm{H}$ & 13 & 055 & (A) & 05/18/06 & DP & l & P06084:01-28 & $\begin{array}{l}\text { Remote visual tank wall inspection } \\
\text { revealed no changes since last } \\
\text { evaluated on } 5 / 28 / 05 \text {. }\end{array}$ \\
\hline $\mathrm{H}$ & 13 & 071 & (A) & 05/18/06 & DP & I & P06085:01-27 & $\begin{array}{l}\text { Remote visual tank wall inspection } \\
\text { revealed no changes since last } \\
\text { evaluated on } 5 / 28 / 05 \text {. }\end{array}$ \\
\hline $\mathrm{H}$ & 13 & 107 & (A) & 05/18/06 & DP & I & P06095:01-27 & $\begin{array}{l}\text { Remote visual tank wall inspection } \\
\text { revealed no changes since last } \\
\text { evaluated on } 5 / 28 / 05 \text {. }\end{array}$ \\
\hline $\mathrm{H}$ & 13 & 151 & (A) & 05/18/06 & DP & l & P06096:01-28 & $\begin{array}{l}\text { Remote visual tank wall inspection } \\
\text { revealed no changes since last } \\
\text { evaluated on } 5 / 28 / 05 \text {. }\end{array}$ \\
\hline $\mathrm{H}$ & 13 & 175 & (A) & $03 / 11 / 06$ & WAP & l & P06029:03 & $\begin{array}{l}\text { Remote visual tank wall inspection } \\
\text { revealed no changes since last } \\
\text { evaluated on } 9 / 13 / 05 \text {. }\end{array}$ \\
\hline $\mathrm{H}$ & 13 & 207 & (A) & 03/11/06 & WAP & l & P06029:04 & $\begin{array}{l}\text { Remote visual tank wall inspection } \\
\text { revealed no changes since last } \\
\text { evaluated on } 6 / 6 / 05 \text {. }\end{array}$ \\
\hline $\mathrm{H}$ & 13 & 228 & (A) & 05/18/06 & DP & l & P06097:01-28 & $\begin{array}{l}\text { Remote visual tank wall inspection } \\
\text { revealed no changes since last } \\
\text { evaluated on } 9 / 12 / 05 \text {. }\end{array}$ \\
\hline $\mathrm{H}$ & 13 & East & (A) & $03 / 11 / 06$ & WAP & l & P06029:01 & $\begin{array}{l}\text { Remote visual tank wall inspection } \\
\text { revealed no changes since last } \\
\text { evaluated on } 11 / 8 / 05 \text {. }\end{array}$ \\
\hline $\mathrm{H}$ & 13 & East & (A) & 05/08/06 & CCTV & l & 1024 & $\begin{array}{l}\text { The annulus jet showed no } \\
\text { degradation per T-DS-G-00043. }\end{array}$ \\
\hline $\mathrm{H}$ & 13 & North & (A) & 05/18/06 & DP & l & P06091:01-28 & $\begin{array}{l}\text { Remote visual tank wall inspection } \\
\text { revealed no changes since last } \\
\text { evaluated on } 5 / 28 / 05 \text {. }\end{array}$ \\
\hline $\mathrm{H}$ & 13 & North & (A) & $05 / 18 / 06$ & DP & l & P06091:18 & $\begin{array}{l}\text { The magnetically mounted tank wall } \\
\text { thermocouple was properly } \\
\text { positioned per T-DS-G-00010. }\end{array}$ \\
\hline
\end{tabular}




\begin{tabular}{|c|c|c|c|c|c|c|c|c|}
\hline \multirow{2}{*}{ AREA } & \multirow{2}{*}{$\begin{array}{c}\begin{array}{c}\text { TANK OR } \\
\text { ANCILLARY }\end{array} \\
13\end{array}$} & \multicolumn{2}{|c|}{$\begin{array}{c}\text { ACCESS OPENING } \\
\text { (A OR I) }\end{array}$} & \multirow{2}{*}{$\frac{\text { DATE }}{03 / 13 / 06}$} & \multicolumn{3}{|c|}{$\begin{array}{l}\text { INSPECTION METHOD } \\
\text { IDENTIFICATION NUMBER }\end{array}$} & \multirow{2}{*}{$\begin{array}{l}\text { REMARKS } \\
\text { Remote visual tank wall inspection } \\
\text { revealed no changes since last } \\
\text { evaluated on } 5 / 28 / 05 \text {. }\end{array}$} \\
\hline & & South & (A) & & $\mathrm{DP}$ & I & P06039:01-28 & \\
\hline $\mathrm{H}$ & 13 & West & (A) & $03 / 11 / 06$ & WAP & I & P06029:02 & $\begin{array}{l}\text { Remote visual tank wall inspection } \\
\text { revealed no changes since last } \\
\text { evaluated on } 6 / 6 / 05 \text {. }\end{array}$ \\
\hline $\mathrm{H}$ & 14 & 013 & (A) & 03/10/06 & WAP & l & P06017:08 & $\begin{array}{l}\text { Remote visual tank wall inspection } \\
\text { revealed no changes since last } \\
\text { evaluated on } 9 / 14 / 05 \text {. The annulus } \\
\text { jet beneath the south riser showed } \\
\text { no degradation per T-DS-G-00043. }\end{array}$ \\
\hline $\mathrm{H}$ & 14 & 032 & (A) & 03/10/06 & WAP & l & P06017:09 & $\begin{array}{l}\text { Remote visual tank wall inspection } \\
\text { revealed no changes since last } \\
\text { evaluated on } 6 / 13 / 05 \text {. }\end{array}$ \\
\hline $\mathrm{H}$ & 14 & 065 & (A) & 03/10/06 & $\mathrm{DP}$ & I & P06020:01-28 & $\begin{array}{l}\text { Remote visual inspection of the } \\
\text { secondary vessel wall revealed no } \\
\text { areas of concern since last evaluated } \\
\text { on } 9 / 15 / 05 \text {. }\end{array}$ \\
\hline $\mathrm{H}$ & 14 & 065 & (A) & 03/10/06 & $\mathrm{DP}$ & I & P06019:01-30 & $\begin{array}{l}\text { Remote visual tank wall inspection } \\
\text { revealed no changes since last } \\
\text { evaluated on } 9 / 15 / 05 \text {. }\end{array}$ \\
\hline $\mathrm{H}$ & 14 & 108 & (A) & 03/10/06 & $\mathrm{DP}$ & l & P06021:01-28 & $\begin{array}{l}\text { Remote visual tank wall inspection } \\
\text { revealed no changes since last } \\
\text { evaluated on } 6 / 13 / 05 \text {. }\end{array}$ \\
\hline $\mathrm{H}$ & 14 & 118 & (A) & 03/10/06 & WAP & I & P06017:01 & $\begin{array}{l}\text { Remote visual tank wall inspection } \\
\text { revealed no changes since last } \\
\text { evaluated on } 9 / 15 / 05 \text {. }\end{array}$ \\
\hline $\mathrm{H}$ & 14 & 125 & (A) & 03/10/06 & $\mathrm{DP}$ & I & P06022:01-21 & $\begin{array}{l}\text { Remote visual tank wall inspection } \\
\text { revealed no changes since last } \\
\text { evaluated on } 6 / 13 / 05 \text {. }\end{array}$ \\
\hline $\mathrm{H}$ & 14 & 151 & (A) & 03/10/06 & WAP & I & P06017:02 & $\begin{array}{l}\text { Remote visual tank wall inspection } \\
\text { revealed no changes since last } \\
\text { evaluated on } 9 / 15 / 05 \text {. }\end{array}$ \\
\hline $\mathrm{H}$ & 14 & 169 & (A) & 03/10/06 & WAP & I & P06017:03 & $\begin{array}{l}\text { Remote visual tank wall inspection } \\
\text { revealed no changes since last } \\
\text { evaluated on } 9 / 15 / 05 \text {. }\end{array}$ \\
\hline $\mathrm{H}$ & 14 & 207 & (A) & 03/10/06 & WAP & I & P06017:05 & $\begin{array}{l}\text { Remote visual tank wall inspection } \\
\text { revealed no changes since last } \\
\text { evaluated on } 9 / 15 / 05 \text {. }\end{array}$ \\
\hline $\mathrm{H}$ & 14 & 235 & (A) & 03/10/06 & WAP & l & P06017:07 & $\begin{array}{l}\text { Remote visual tank wall inspection } \\
\text { revealed no changes since last } \\
\text { evaluated on } 9 / 14 / 05 \text {. }\end{array}$ \\
\hline
\end{tabular}




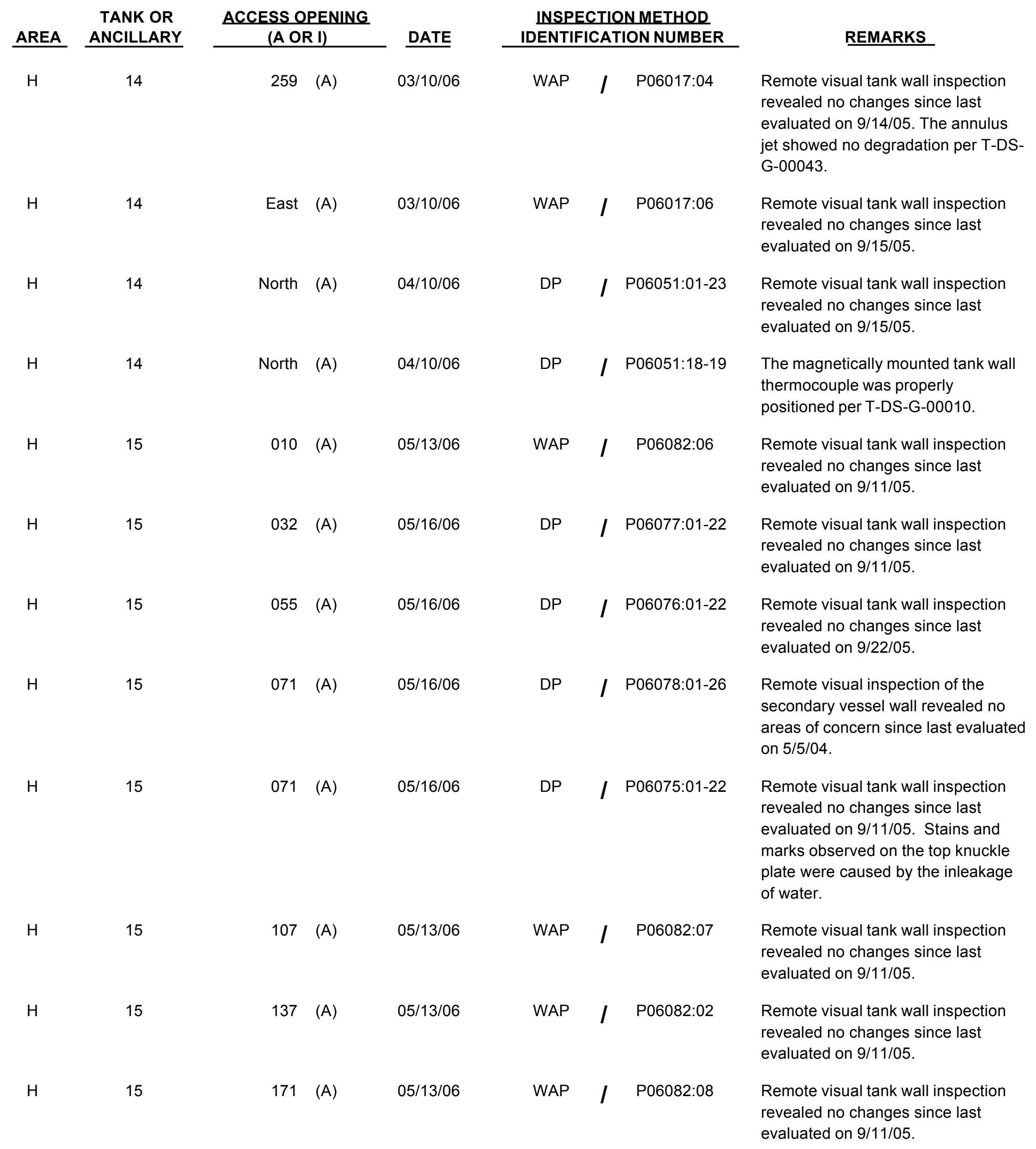




\begin{tabular}{|c|c|c|c|c|c|c|c|c|}
\hline \multirow{2}{*}{ AREA } & \multirow{2}{*}{ 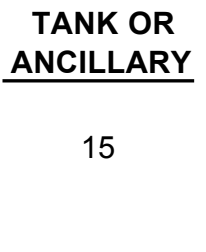 } & \multicolumn{2}{|c|}{$\frac{\text { ACCESS OPENING }}{(A \text { OR I) }}$} & \multirow{2}{*}{$\frac{\text { DATE }}{05 / 13 / 06}$} & \multicolumn{3}{|c|}{$\begin{array}{l}\text { INSPECTION METHOD } \\
\text { IDENTIFICATION NUMBER }\end{array}$} & \multirow{2}{*}{$\begin{array}{l}\qquad \text { REMARKS } \\
\text { Remote visual tank wall inspection } \\
\text { revealed no changes since last } \\
\text { evaluated on } 9 / 11 / 05 \text {. }\end{array}$} \\
\hline & & 182 & (A) & & WAP & l & P06082:03 & \\
\hline $\mathrm{H}$ & 15 & 207 & (A) & 05/16/06 & DP & I & P06074:01-24 & $\begin{array}{l}\text { Remote visual tank wall inspection } \\
\text { revealed no changes since last } \\
\text { evaluated on } 9 / 11 / 05 \text {. The annulus } \\
\text { jet beneath the east riser showed no } \\
\text { degradation per T-DS-G-00043. }\end{array}$ \\
\hline $\mathrm{H}$ & 15 & 223 & (A) & 05/16/06 & DP & I & P06081:01-22 & $\begin{array}{l}\text { Remote visual tank wall inspection } \\
\text { revealed no changes since last } \\
\text { evaluated on } 9 / 11 / 05 \text {. }\end{array}$ \\
\hline $\mathrm{H}$ & 15 & 242 & (A) & $10 / 30 / 06$ & CCTV & l & 1370 & $\begin{array}{l}\text { The magnetically mounted tank wall } \\
\text { thermocouple was properly } \\
\text { positioned per T-DS-G-00010. }\end{array}$ \\
\hline $\mathrm{H}$ & 15 & East & (A) & 05/13/06 & WAP & l & P06082:04 & $\begin{array}{l}\text { Remote visual tank wall inspection } \\
\text { revealed no changes since last } \\
\text { evaluated on } 9 / 11 / 05 \text {. The annulus } \\
\text { jet showed no degradation per T-DS- } \\
\text { G-00043. }\end{array}$ \\
\hline $\mathrm{H}$ & 15 & North & (A) & 05/16/06 & DP & I & P06080:01-22 & $\begin{array}{l}\text { Remote visual tank wall inspection } \\
\text { revealed no changes since last } \\
\text { evaluated on } 9 / 11 / 05 \text {. }\end{array}$ \\
\hline $\mathrm{H}$ & 15 & South & (A) & 05/13/06 & WAP & I & P06082:05 & $\begin{array}{l}\text { Remote visual tank wall inspection } \\
\text { revealed no changes since last } \\
\text { evaluated on } 9 / 11 / 05 \text {. }\end{array}$ \\
\hline $\mathrm{H}$ & 15 & West & (A) & 05/16/06 & DP & I & P06079:01-22 & $\begin{array}{l}\text { Remote visual tank wall inspection } \\
\text { revealed no changes since last } \\
\text { evaluated on } 9 / 11 / 05 \text {. }\end{array}$ \\
\hline $\mathrm{H}$ & 16 & 035 & (A) & $12 / 06 / 06$ & WAP & I & P06283:05 & $\begin{array}{l}\text { Remote visual tank wall inspection } \\
\text { revealed no changes since last } \\
\text { evaluated on } 9 / 25 / 06 \text {. }\end{array}$ \\
\hline $\mathrm{H}$ & 16 & 118 & (A) & $11 / 14 / 06$ & WAP & I & P06283:03 & $\begin{array}{l}\text { Remote visual tank wall inspection } \\
\text { revealed no changes since last } \\
\text { evaluated on } 9 / 25 / 05 \text {. }\end{array}$ \\
\hline $\mathrm{H}$ & 16 & 207 & (A) & $11 / 14 / 06$ & WAP & l & P06283:02 & $\begin{array}{l}\text { Remote visual tank wall inspection } \\
\text { revealed no changes since last } \\
\text { evaluated on } 9 / 25 / 05 \text {. }\end{array}$ \\
\hline $\mathrm{H}$ & 16 & 262 & (A) & $11 / 14 / 06$ & WAP & l & P06283:04 & $\begin{array}{l}\text { Remote visual tank wall inspection } \\
\text { revealed no changes since last } \\
\text { evaluated on } 6 / 20 / 05 \text {. }\end{array}$ \\
\hline $\mathrm{H}$ & 16 & East & (A) & $11 / 14 / 06$ & WAP & I & P06283:01 & $\begin{array}{l}\text { Remote visual tank wall inspection } \\
\text { revealed no changes since last } \\
\text { evaluated on } 9 / 25 / 05 \text {. }\end{array}$ \\
\hline
\end{tabular}




\begin{tabular}{|c|c|c|c|c|c|c|c|c|}
\hline$\frac{\text { AREA }}{\mathrm{H}}$ & $\begin{array}{c}\begin{array}{c}\text { TANK OR } \\
\text { ANCILLARY }\end{array} \\
16\end{array}$ & \multicolumn{2}{|c|}{$\frac{\text { ACCESS OPENING }}{(\text { A OR I) }}$} & $\begin{array}{c}\text { DATE } \\
04 / 27 / 06\end{array}$ & \multicolumn{3}{|c|}{$\begin{array}{l}\text { INSPECTION METHOD } \\
\text { IDENTIFICATION NUMBER }\end{array}$} & \multirow{2}{*}{$\begin{array}{l}\quad \text { REMARKS } \\
\text { Remote visual tank wall inspection } \\
\text { revealed no changes since last } \\
\text { evaluated on } 9 / 25 / 05 \text {. Leaked waste } \\
\text { on the annulus floor had been } \\
\text { redistributed due to the inleakage of } \\
\text { water. }\end{array}$} \\
\hline & 16 & West & (A) & 04/27/06 & DP & l & P06053:01-29 & \\
\hline$F$ & 18 & Center & (I) & 04/29/06 & CCTV & l & 1374 & $\begin{array}{l}\text { Remote visual inspection of the tank } \\
\text { wall and concrete dome revealed no } \\
\text { areas of concern since last evaluated } \\
\text { on } 3 / 29 / 05 \text {. Damp areas on the } \\
\text { concrete dome were caused by the } \\
\text { inleakage of water around the risers. } \\
\text { Liquid was observed throughout } \\
\text { most of the tank with some exposed } \\
\text { solids. The HLLCP was properly } \\
\text { deployed. }\end{array}$ \\
\hline $\mathrm{F}$ & 19 & Center & (I) & $04 / 30 / 06$ & CCTV & l & 1375 & $\begin{array}{l}\text { Remote visual inspection of the tank } \\
\text { wall and concrete dome showed no } \\
\text { areas of concern since last evaluated } \\
\text { on } 3 / 30 / 05 \text {. Damp areas on the } \\
\text { concrete dome were caused by the } \\
\text { inleakage of water around the risers. }\end{array}$ \\
\hline $\mathrm{F}$ & 19 & Center & (I) & $11 / 14 / 06$ & CCTV & l & 1506 & $\begin{array}{l}\text { Remote visual inspection of the tank } \\
\text { wall and concrete dome revealed no } \\
\text { areas of concern since last evaluated } \\
\text { on } 3 / 30 / 05 \text {. Damp areas on the } \\
\text { concrete dome were caused by the } \\
\text { inleakage of water around the risers. }\end{array}$ \\
\hline $\mathrm{H}$ & 21 & NE & (I) & $11 / 03 / 06$ & CCTV & I & 1427 & $\begin{array}{l}\text { Remote visual inspection of the tank } \\
\text { wall and concrete dome revealed no } \\
\text { areas of concern since last evaluated } \\
\text { on } 9 / 29 / 05 \text {. Calciferous deposits on } \\
\text { the dome indicate that water } \\
\text { continues to leak through the } \\
\text { concrete dome. The HLLCP was } \\
\text { properly deployed. }\end{array}$ \\
\hline $\mathrm{H}$ & 22 & NW & (I) & $11 / 05 / 06$ & CCTV & I & 1500 & $\begin{array}{l}\text { Remote visual inspection of the tank } \\
\text { wall and concrete dome revealed no } \\
\text { areas of concern since last evaluated } \\
\text { on } 3 / 8 / 05 \text {. Stains observed on the } \\
\text { concrete dome were caused by } \\
\text { water leaking through the dome and } \\
\text { around the risers. The HLLCP was } \\
\text { properly deployed. }\end{array}$ \\
\hline
\end{tabular}




\begin{tabular}{|c|c|c|c|c|c|c|c|}
\hline \multirow{2}{*}{$\frac{\text { AREA }}{\mathrm{H}}$} & \multirow{2}{*}{$\begin{array}{c}\begin{array}{c}\text { TANK OR } \\
\text { ANCILLARY }\end{array} \\
23\end{array}$} & $\frac{\text { ACCESS OPENING }}{(\text { A OR I) }}$ & $\frac{\text { DATE }}{12 / 10 / 06}$ & \multicolumn{3}{|c|}{$\begin{array}{l}\text { INSPECTION METHOD } \\
\text { IDENTIFICATION NUMBER }\end{array}$} & \multirow{2}{*}{$\begin{array}{l}\text { REMARKS } \\
\text { Remote visual inspection of the tank } \\
\text { wall and concrete dome revealed no } \\
\text { areas of concern since last evaluated } \\
\text { on } 3 / 8 / 05 \text {. Stains observed on the } \\
\text { concrete dome were caused by } \\
\text { water leaking through the dome and } \\
\text { around the risers. The HLLCP was } \\
\text { properly deployed. }\end{array}$} \\
\hline & & SW (I) & $12 / 10 / 06$ & CCTV & T & 1345 & \\
\hline
\end{tabular}

$\mathrm{H}$

$\mathrm{F}$

$\mathrm{F}$

$\mathrm{F}$
24

25

25

25

25

25

25

F

F

NW (I)

$\mathrm{A}-01 \quad(\mathrm{~A})$

A-02 (A)

A-03 (A)

25

F

25

25
A-04 (A)

$\mathrm{P}-01 \quad(\mathrm{~A})$

$\mathrm{P}-02 \quad(\mathrm{~A})$

$\mathrm{P}-03 \quad(\mathrm{~A})$

P-04 (A)

P-05 (A)
$11 / 19 / 06$

$05 / 12 / 06$

$05 / 12 / 06$

07/18/06

$05 / 12 / 06$

$05 / 12 / 06$

05/12/06

$05 / 12 / 06$

$05 / 12 / 06$

$05 / 13 / 06$
CCTV / 1282

WAP I P06073:02

WAP

I P06073:06

WAP

I

P06197:02

WAP

I P06073:11

WAP

I P06073:01

WAP

I P06073:03

WAP

I P06073:04

WAP

(

WAP

DP
I P06073:05

Remote visual inspection of the tank wall and concrete dome revealed no changes since last evaluated on 9/29/05. The HLLCP was properly positioned.

Remote visual tank wall inspection revealed no areas of concern since last evaluated on 2/25/05.

Remote visual tank wall inspection revealed no areas of concern since last evaluated on 2/25/05.

Remote visual tank wall inspection revealed no areas of concern since last evaluated on $2 / 25 / 05$. The annulus jet beneath the $F$ riser showed no degradation per T-DS-G -00043 .

Remote visual tank wall inspection revealed no areas of concern since last evaluated on 2/25/05.

Remote visual tank wall inspection revealed no areas of concern since last evaluated on 2/26/05.

Remote visual tank wall inspection revealed no areas of concern since last evaluated on 2/26/05.

Remote visual tank wall inspection revealed no areas of concern since last evaluated on 2/25/05.

Remote visual tank wall inspection revealed no areas of concern since last evaluated on 2/25/05.

Remote visual tank wall inspection revealed no areas of concern since last evaluated on 2/25/05. 


\begin{tabular}{|c|c|c|c|c|c|c|c|c|}
\hline \multirow{2}{*}{$\frac{\text { AREA }}{F}$} & \multirow{2}{*}{$\begin{array}{c}\begin{array}{c}\text { TANK OR } \\
\text { ANCILLARY }\end{array} \\
25\end{array}$} & \multicolumn{2}{|c|}{$\frac{\text { ACCESS OPENING }}{(A \text { OR I) }}$} & \multirow{2}{*}{$\begin{array}{l}\text { DATE } \\
05 / 13 / 06\end{array}$} & \multicolumn{3}{|c|}{$\begin{array}{l}\text { INSPECTION METHOD } \\
\text { IDENTIFICATION NUMBER }\end{array}$} & \multirow{2}{*}{$\begin{array}{l}\text { REMARKS } \\
\text { Remote visual inspection of the } \\
\text { secondary vessel wall revealed no } \\
\text { areas of concern since last evaluated } \\
\text { on } 2 / 26 / 05 \text {. }\end{array}$} \\
\hline & & P-06 & (A) & & DP & I & P06069:01-27 & \\
\hline$F$ & 25 & P-06 & (A) & $05 / 13 / 06$ & DP & I & P06070:01-27 & $\begin{array}{l}\text { Remote visual tank wall inspection } \\
\text { revealed no areas of concern since } \\
\text { last evaluated on } 2 / 25 / 05 \text {. }\end{array}$ \\
\hline $\mathrm{F}$ & 25 & P-07 & (A) & 05/12/06 & WAP & I & P06073:07 & $\begin{array}{l}\text { Remote visual tank wall inspection } \\
\text { revealed no areas of concern since } \\
\text { last evaluated on } 2 / 26 / 05 \text {. }\end{array}$ \\
\hline $\mathrm{F}$ & 25 & P-08 & (A) & $05 / 12 / 06$ & WAP & I & P06073:08 & $\begin{array}{l}\text { Remote visual tank wall inspection } \\
\text { revealed no areas of concern since } \\
\text { last evaluated on } 2 / 26 / 05 \text {. }\end{array}$ \\
\hline $\mathrm{F}$ & 25 & $P-10$ & (A) & $06 / 18 / 06$ & DP & I & P06168:01-27 & $\begin{array}{l}\text { Remote visual tank wall inspection } \\
\text { revealed no areas of concern since } \\
\text { last evaluated on } 2 / 25 / 05 \text {. }\end{array}$ \\
\hline $\mathrm{F}$ & 25 & $\mathrm{P}-11$ & (A) & 07/18/06 & WAP & I & P06197:01 & $\begin{array}{l}\text { Remote visual tank wall inspection } \\
\text { revealed no areas of concern since } \\
\text { last evaluated on } 2 / 25 / 05 \text {. }\end{array}$ \\
\hline$F$ & 25 & $\mathrm{P}-12$ & (A) & $05 / 12 / 06$ & WAP & I & P06073:12 & $\begin{array}{l}\text { Remote visual tank wall inspection } \\
\text { revealed no areas of concern since } \\
\text { last evaluated on } 2 / 25 / 05 \text {. }\end{array}$ \\
\hline$F$ & 25 & $\mathrm{P}-12$ & (A) & 05/13/06 & DP & I & P06068:01-27 & $\begin{array}{l}\text { Remote visual tank wall inspection } \\
\text { revealed no areas of concern since } \\
\text { last evaluated on } 2 / 25 / 05 \text {. }\end{array}$ \\
\hline$F$ & 25 & $\mathrm{P}-13$ & (A) & $05 / 13 / 06$ & DP & I & P06071:01-27 & $\begin{array}{l}\text { Remote visual tank wall inspection } \\
\text { revealed no areas of concern since } \\
\text { last evaluated on } 2 / 25 / 05 \text {. }\end{array}$ \\
\hline$F$ & 25 & P-14 & (A) & 05/13/06 & DP & I & P06067:01-27 & $\begin{array}{l}\text { Remote visual tank wall inspection } \\
\text { revealed no areas of concern since } \\
\text { last evaluated on } 2 / 25 / 05 \text {. }\end{array}$ \\
\hline$F$ & 26 & $A-01$ & (A) & $05 / 12 / 06$ & WAP & I & P06090:02 & $\begin{array}{l}\text { Remote visual tank wall inspection } \\
\text { revealed no areas of concern since } \\
\text { last evaluated on } 2 / 25 / 05 \text {. }\end{array}$ \\
\hline $\mathrm{F}$ & 26 & $\mathrm{~A}-02$ & (A) & $05 / 12 / 06$ & WAP & I & P06090:06 & $\begin{array}{l}\text { Remote visual tank wall inspection } \\
\text { revealed no areas of concern since } \\
\text { last evaluated on } 2 / 25 / 05 \text {. }\end{array}$ \\
\hline$F$ & 26 & A-02 & (A) & $11 / 17 / 06$ & CCTV & I & 1505 & $\begin{array}{l}\text { The conductivity probe was properly } \\
\text { deployed per J-JX-G-0001. }\end{array}$ \\
\hline $\mathrm{F}$ & 26 & $\mathrm{~A}-03$ & (A) & $05 / 12 / 06$ & WAP & I & P06090:09 & $\begin{array}{l}\text { Remote visual tank wall inspection } \\
\text { revealed no areas of concern since } \\
\text { last evaluated on } 2 / 25 / 05 \text {. }\end{array}$ \\
\hline
\end{tabular}




\begin{tabular}{|c|c|c|c|c|c|c|c|c|}
\hline \multirow{2}{*}{ AREA } & \multirow{2}{*}{$\begin{array}{c}\text { TANK OR } \\
\text { ANCILLARY } \\
26\end{array}$} & \multicolumn{2}{|c|}{$\begin{array}{c}\text { ACCESS OPENING } \\
\text { (A OR I) }\end{array}$} & \multirow{2}{*}{$\frac{\text { DATE }}{12 / 04 / 06}$} & \multicolumn{3}{|c|}{$\begin{array}{l}\text { INSPECTION METHOD } \\
\text { IDENTIFICATION NUMBER }\end{array}$} & \multirow{2}{*}{$\begin{array}{l}\text { REMARKS } \\
\text { The conductivity probe was properly } \\
\text { deployed per J-JX-G-0001. }\end{array}$} \\
\hline & & A-03 & (A) & & CCTV & I & 1520 & \\
\hline $\mathrm{F}$ & 26 & A-04 & (A) & $05 / 12 / 06$ & WAP & I & P06090:12 & $\begin{array}{l}\text { Remote visual tank wall inspection } \\
\text { revealed no areas of concern since } \\
\text { last evaluated on } 2 / 25 / 05 \text {. }\end{array}$ \\
\hline $\mathrm{F}$ & 26 & A-04 & (A) & $11 / 19 / 06$ & CCTV & l & 1499 & $\begin{array}{l}\text { The conductivity probe was properly } \\
\text { deployed per J-JX-G-0001. }\end{array}$ \\
\hline $\mathrm{F}$ & 26 & P-01 & (A) & $05 / 12 / 06$ & WAP & I & P06090:01 & $\begin{array}{l}\text { Remote visual tank wall inspection } \\
\text { revealed no areas of concern since } \\
\text { last evaluated on } 2 / 25 / 05 \text {. }\end{array}$ \\
\hline $\mathrm{F}$ & 26 & P-02 & (A) & $05 / 12 / 06$ & WAP & I & P06090:03 & $\begin{array}{l}\text { Remote visual tank wall inspection } \\
\text { revealed no areas of concern since } \\
\text { last evaluated on } 2 / 25 / 05 \text {. }\end{array}$ \\
\hline $\mathrm{F}$ & 26 & P-03 & (A) & $05 / 12 / 06$ & WAP & I & P06090:04 & $\begin{array}{l}\text { Remote visual tank wall inspection } \\
\text { revealed no areas of concern since } \\
\text { last evaluated on } 2 / 25 / 05 \text {. }\end{array}$ \\
\hline $\mathrm{F}$ & 26 & P-04 & (A) & $05 / 12 / 06$ & WAP & l & P06090:05 & $\begin{array}{l}\text { Remote visual tank wall inspection } \\
\text { revealed no areas of concern since } \\
\text { last evaluated on } 2 / 25 / 05 \text {. }\end{array}$ \\
\hline $\mathrm{F}$ & 26 & P-05 & (A) & 05/20/06 & DP & I & P06139:01-27 & $\begin{array}{l}\text { Remote visual tank wall inspection } \\
\text { revealed no areas of concern since } \\
\text { last evaluated on } 2 / 25 / 05 \text {. }\end{array}$ \\
\hline $\mathrm{F}$ & 26 & P-06 & (A) & 05/20/06 & DP & I & P06141:01-27 & $\begin{array}{l}\text { Remote visual inspection of the } \\
\text { secondary vessel wall revealed no } \\
\text { areas of concern since last evaluated } \\
\text { on } 2 / 25 / 05 \text {. }\end{array}$ \\
\hline $\mathrm{F}$ & 26 & P-06 & (A) & $05 / 20 / 06$ & DP & I & P06140:01-25 & $\begin{array}{l}\text { Remote visual tank wall inspection } \\
\text { revealed no areas of concern since } \\
\text { last evaluated on } 2 / 25 / 05 \text {. }\end{array}$ \\
\hline $\mathrm{F}$ & 26 & P-07 & (A) & 05/12/06 & WAP & I & P06090:07 & $\begin{array}{l}\text { Remote visual tank wall inspection } \\
\text { revealed no areas of concern since } \\
\text { last evaluated on } 2 / 25 / 05 \text {. }\end{array}$ \\
\hline $\mathrm{F}$ & 26 & P-08 & (A) & $05 / 12 / 06$ & WAP & I & P06090:08 & $\begin{array}{l}\text { Remote visual tank wall inspection } \\
\text { revealed no areas of concern since } \\
\text { last evaluated on } 2 / 25 / 05 \text {. }\end{array}$ \\
\hline $\mathrm{F}$ & 26 & P-09 & (A) & 05/12/06 & WAP & l & P06090:13 & $\begin{array}{l}\text { Remote visual tank wall inspection } \\
\text { revealed no areas of concern since } \\
\text { last evaluated on } 2 / 25 / 05 \text {. The } \\
\text { annulus jet beneath the F riser } \\
\text { showed no degradation per T-DS-G } \\
\text { - } 00043 \text {. }\end{array}$ \\
\hline
\end{tabular}




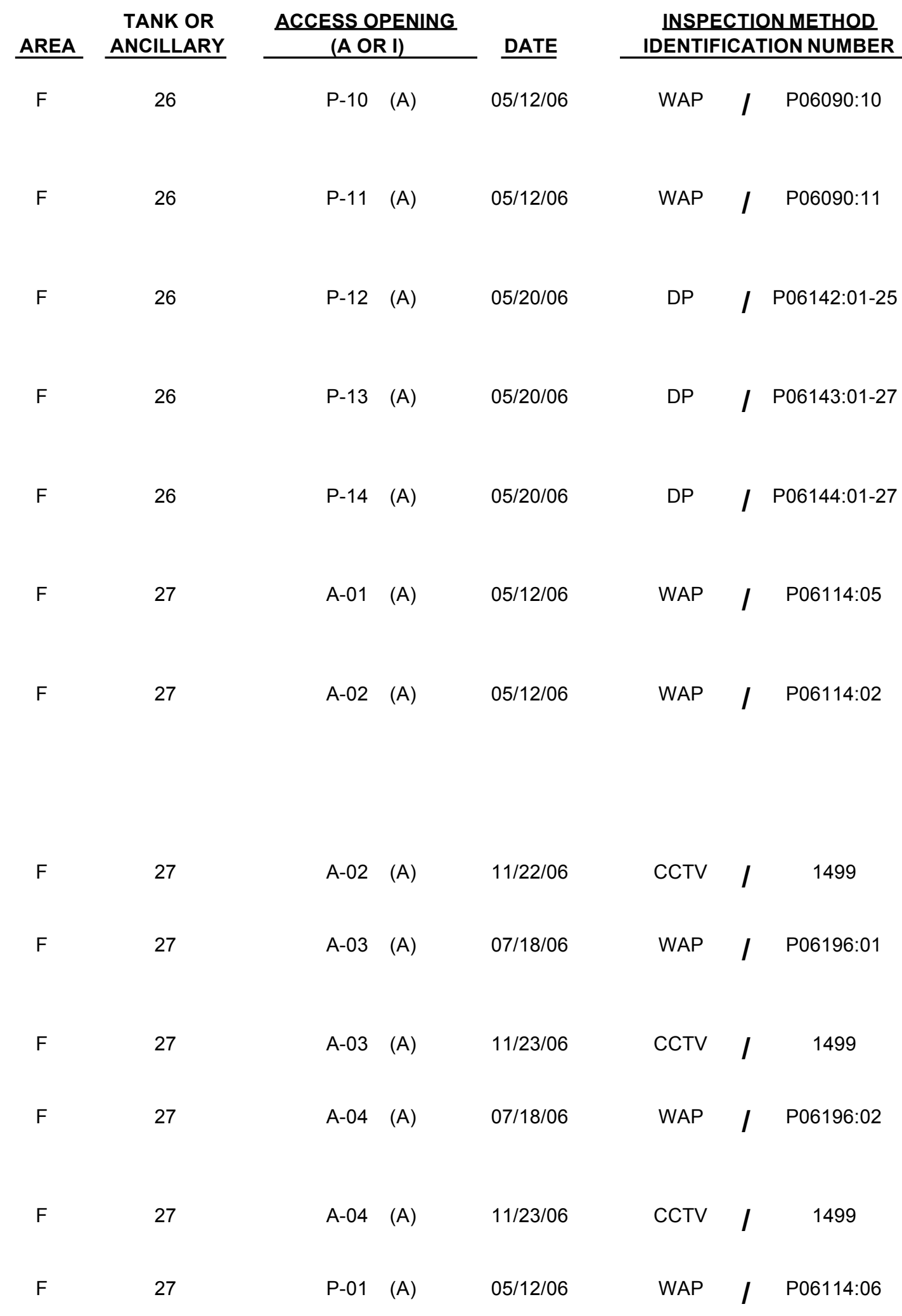

\section{REMARKS}

Remote visual tank wall inspection revealed no areas of concern since last evaluated on 2/25/05.

Remote visual tank wall inspection revealed no areas of concern since last evaluated on 2/25/05.

Remote visual tank wall inspection revealed no areas of concern since last evaluated on 2/25/05.

Remote visual tank wall inspection revealed no areas of concern since last evaluated on 2/25/05.

Remote visual tank wall inspection revealed no areas of concern since last evaluated on 2/25/05.

Remote visual tank wall inspection revealed no areas of concern since last evaluated on 2/25/05.

Remote visual tank wall inspection revealed no areas of concern since last evaluated on $2 / 25 / 05$. Stains and marks observed on the secondary vessel wall were caused by the inleakage of water.

The conductivity probe was properly deployed per J-JX-G-0001.

Remote visual tank wall inspection revealed no areas of concern since last evaluated on 2/25/05.

The conductivity probe was properly deployed per J-JX-G-0001.

Remote visual tank wall inspection revealed no areas of concern since last evaluated on 2/25/05.

The conductivity probe was properly deployed per J-JX-G-0001.

Remote visual tank wall inspection revealed no areas of concern since last evaluated on $2 / 26 / 05$. The annulus jet beneath the $F$ riser showed no degradation per T-DS-G -00043 . 


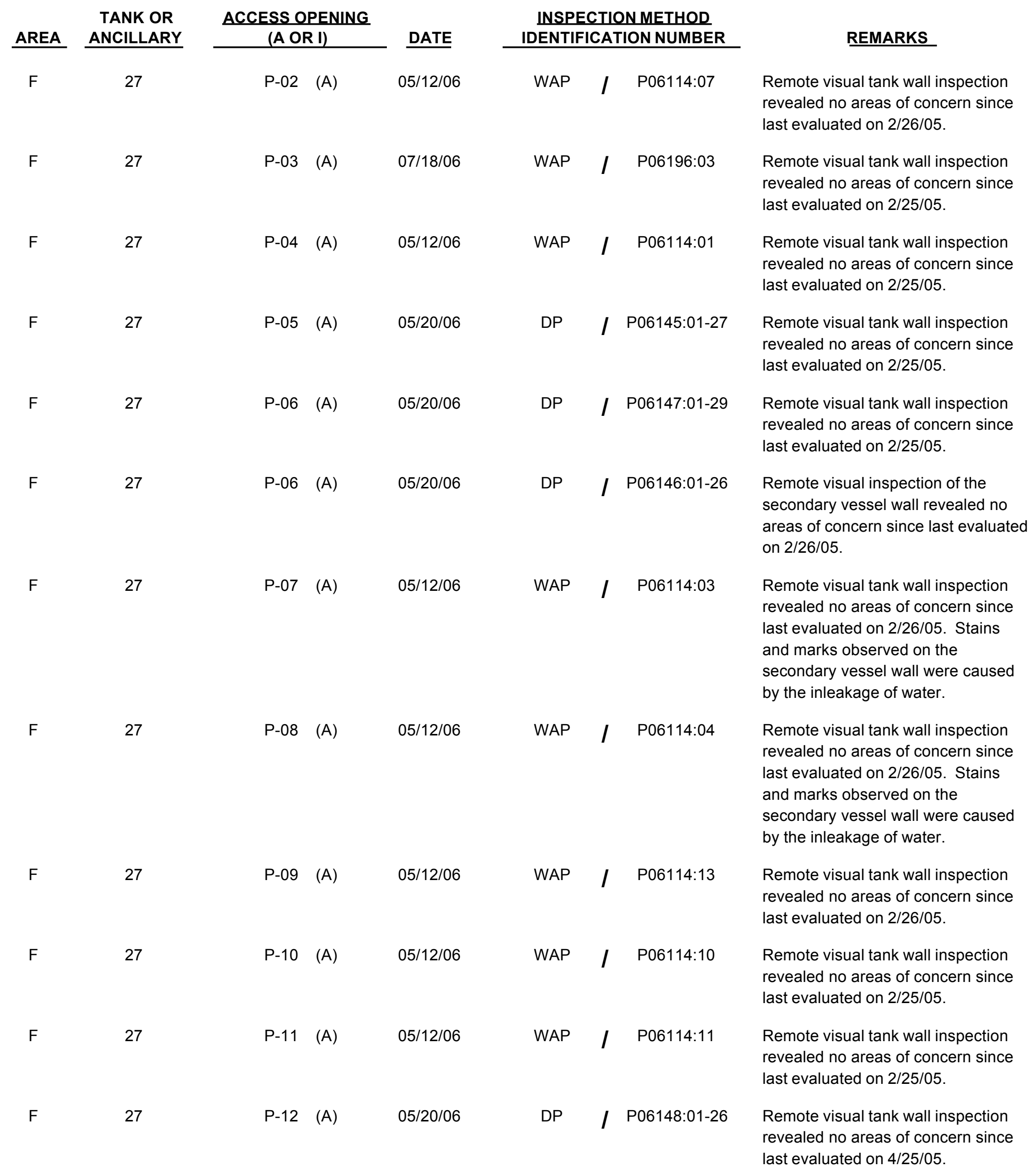




\begin{tabular}{|c|c|c|c|c|c|c|c|c|}
\hline \multirow{2}{*}{ AREA } & \multirow{2}{*}{$\begin{array}{c}\text { TANK OR } \\
\text { ANCILLARY } \\
27\end{array}$} & \multicolumn{2}{|c|}{$\frac{\text { ACCESS OPENING }}{(\text { A OR I) }}$} & \multirow{2}{*}{$\begin{array}{l}\text { DATE } \\
\text { 05/20/06 }\end{array}$} & \multicolumn{3}{|c|}{$\begin{array}{l}\text { INSPECTION METHOD } \\
\text { IDENTIFICATION NUMBER }\end{array}$} & \multirow{2}{*}{$\begin{array}{l}\text { REMARKS } \\
\text { Remote visual tank wall inspection } \\
\text { revealed no areas of concern since } \\
\text { last evaluated on } 2 / 25 / 05 \text {. }\end{array}$} \\
\hline & & P-13 & (A) & & DP & I & P06149:01-28 & \\
\hline $\mathrm{F}$ & 27 & P-14 & (A) & 05/20/06 & DP & I & P06150:01-26 & $\begin{array}{l}\text { Remote visual tank wall inspection } \\
\text { revealed no areas of concern since } \\
\text { last evaluated on } 2 / 25 / 05 \text {. }\end{array}$ \\
\hline $\mathrm{F}$ & 28 & $A-01$ & (A) & 05/12/06 & WAP & l & P06089:01 & $\begin{array}{l}\text { Remote visual tank wall inspection } \\
\text { revealed no areas of concern since } \\
\text { last evaluated on } 2 / 25 / 05 \text {. }\end{array}$ \\
\hline $\mathrm{F}$ & 28 & A-02 & (A) & 05/12/06 & WAP & l & P06089:10 & $\begin{array}{l}\text { Remote visual tank wall inspection } \\
\text { revealed no areas of concern since } \\
\text { last evaluated on } 2 / 25 / 05 \text {. }\end{array}$ \\
\hline $\mathrm{F}$ & 28 & A-02 & (A) & $12 / 07 / 06$ & CCTV & l & 1520 & $\begin{array}{l}\text { The conductivity probe was properly } \\
\text { deployed per J-JX-G-0001. }\end{array}$ \\
\hline $\mathrm{F}$ & 28 & A-03 & (A) & $05 / 12 / 06$ & WAP & l & P06089:05 & $\begin{array}{l}\text { Remote visual tank wall inspection } \\
\text { revealed no areas of concern since } \\
\text { last evaluated on } 2 / 25 / 05 \text {. }\end{array}$ \\
\hline $\mathrm{F}$ & 28 & A-03 & (A) & $12 / 07 / 06$ & CCTV & l & 1520 & $\begin{array}{l}\text { The conductivity probe was properly } \\
\text { deployed per J-JX-G-0001. }\end{array}$ \\
\hline $\mathrm{F}$ & 28 & A-04 & (A) & $05 / 12 / 06$ & WAP & l & P06089:07 & $\begin{array}{l}\text { Remote visual tank wall inspection } \\
\text { revealed no areas of concern since } \\
\text { last evaluated on } 2 / 25 / 05 \text {. }\end{array}$ \\
\hline $\mathrm{F}$ & 28 & A-04 & (A) & $12 / 06 / 06$ & CCTV & l & 1523 & $\begin{array}{l}\text { The conductivity probe was properly } \\
\text { deployed per J-JX-G-0001. }\end{array}$ \\
\hline $\mathrm{F}$ & 28 & P-01 & (A) & $05 / 12 / 06$ & WAP & I & P06089:02 & $\begin{array}{l}\text { Remote visual tank wall inspection } \\
\text { revealed no areas of concern since } \\
\text { last evaluated on } 2 / 25 / 05 \text {. The } \\
\text { annulus jet beneath the } F \text { riser } \\
\text { showed no degradation per T-DS-G } \\
-00043 \text {. }\end{array}$ \\
\hline $\mathrm{F}$ & 28 & P-02 & $(\mathrm{A})$ & 05/20/06 & DP & I & P06127:01-27 & $\begin{array}{l}\text { Remote visual tank wall inspection } \\
\text { revealed no areas of concern since } \\
\text { last evaluated on } 2 / 25 / 05 \text {. }\end{array}$ \\
\hline $\mathrm{F}$ & 28 & P-03 & (A) & $05 / 12 / 06$ & WAP & l & P06089:03 & $\begin{array}{l}\text { Remote visual tank wall inspection } \\
\text { revealed no areas of concern since } \\
\text { last evaluated on } 3 / 2 / 05 \text {. }\end{array}$ \\
\hline $\mathrm{F}$ & 28 & P-04 & (A) & $05 / 12 / 06$ & WAP & l & P06089:04 & $\begin{array}{l}\text { Remote visual tank wall inspection } \\
\text { revealed no areas of concern since } \\
\text { last evaluated on } 2 / 25 / 05 \text {. }\end{array}$ \\
\hline $\mathrm{F}$ & 28 & P-05 & (A) & 05/20/06 & DP & l & P06128:01-27 & $\begin{array}{l}\text { Remote visual tank wall inspection } \\
\text { revealed no areas of concern since } \\
\text { last evaluated on } 2 / 25 / 05 \text {. }\end{array}$ \\
\hline
\end{tabular}




\begin{tabular}{|c|c|c|c|c|c|c|c|c|}
\hline \multirow{2}{*}{ AREA } & \multirow{2}{*}{$\begin{array}{c}\text { TANK OR } \\
\text { ANCILLARY } \\
28\end{array}$} & \multicolumn{2}{|c|}{$\frac{\text { ACCESS OPENING }}{(A \text { OR I) }}$} & \multirow{2}{*}{$\begin{array}{c}\text { DATE } \\
05 / 12 / 06\end{array}$} & \multicolumn{3}{|c|}{$\begin{array}{l}\text { INSPECTION METHOD } \\
\text { IDENTIFICATION NUMBER }\end{array}$} & \multirow{2}{*}{$\begin{array}{l}\qquad \text { REMARKS } \\
\text { Remote visual tank wall inspection } \\
\text { revealed no areas of concern since } \\
\text { last evaluated on } 3 / 2 / 05 \text {. }\end{array}$} \\
\hline & & P-06 & (A) & & WAP & l & P06089:09 & \\
\hline $\mathrm{F}$ & 28 & P-07 & (A) & $05 / 12 / 06$ & WAP & l & P06089:11 & $\begin{array}{l}\text { Remote visual tank wall inspection } \\
\text { revealed no areas of concern since } \\
\text { last evaluated on } 2 / 25 / 05 \text {. }\end{array}$ \\
\hline $\mathrm{F}$ & 28 & P-08 & (A) & $05 / 12 / 06$ & WAP & l & P06089:12 & $\begin{array}{l}\text { Remote visual tank wall inspection } \\
\text { revealed no areas of concern since } \\
\text { last evaluated on } 3 / 2 / 05 \text {. }\end{array}$ \\
\hline$F$ & 28 & P-09 & (A) & 05/20/06 & DP & l & P06129:01-27 & $\begin{array}{l}\text { Remote visual tank wall inspection } \\
\text { revealed no areas of concern since } \\
\text { last evaluated on } 2 / 25 / 05 \text {. }\end{array}$ \\
\hline $\mathrm{F}$ & 28 & P-09 & (A) & 05/20/06 & DP & l & P06130:01-27 & $\begin{array}{l}\text { Remote visual inspection of the } \\
\text { secondary vessel wall revealed no } \\
\text { areas of concern since last evaluated } \\
\text { on } 3 / 2 / 05 \text {. }\end{array}$ \\
\hline $\mathrm{F}$ & 28 & P-10 & (A) & 05/20/06 & DP & I & P06131:01-27 & $\begin{array}{l}\text { Remote visual tank wall inspection } \\
\text { revealed no areas of concern since } \\
\text { last evaluated on } 2 / 25 / 05 \text {. }\end{array}$ \\
\hline $\mathrm{F}$ & 28 & $\mathrm{P}-11$ & (A) & 05/12/06 & WAP & l & P06089:06 & $\begin{array}{l}\text { Remote visual tank wall inspection } \\
\text { revealed no areas of concern since } \\
\text { last evaluated on } 2 / 25 / 05 \text {. }\end{array}$ \\
\hline $\mathrm{F}$ & 28 & $\mathrm{P}-12$ & (A) & $05 / 12 / 06$ & WAP & l & P06089:08 & $\begin{array}{l}\text { Remote visual tank wall inspection } \\
\text { revealed no areas of concern since } \\
\text { last evaluated on } 3 / 2 / 05 \text {. }\end{array}$ \\
\hline $\mathrm{F}$ & 28 & $P-13$ & (A) & 05/20/06 & $\mathrm{DP}$ & l & P06132:01-27 & $\begin{array}{l}\text { Remote visual tank wall inspection } \\
\text { revealed no areas of concern since } \\
\text { last evaluated on } 2 / 25 / 05 \text {. }\end{array}$ \\
\hline$F$ & 28 & $\mathrm{P}-14$ & (A) & 05/20/06 & DP & I & P06133:01-27 & $\begin{array}{l}\text { Remote visual tank wall inspection } \\
\text { revealed no areas of concern since } \\
\text { last evaluated on } 2 / 25 / 05 \text {. }\end{array}$ \\
\hline $\mathrm{H}$ & 29 & A-01 & (A) & 03/07/06 & WAP & l & P06040:06 & $\begin{array}{l}\text { Remote visual tank wall inspection } \\
\text { revealed no areas of concern since } \\
\text { last evaluated on } 1 / 7 / 05 \text {. The } \\
\text { annulus jet showed no degradation } \\
\text { per T-DS-G-00043. }\end{array}$ \\
\hline $\mathrm{H}$ & 29 & A-02 & (A) & 03/07/06 & WAP & l & P06040:03 & $\begin{array}{l}\text { Remote visual tank wall inspection } \\
\text { revealed no areas of concern since } \\
\text { last evaluated on } 1 / 7 / 05 \text {. An } \\
\text { increase in stains and marks } \\
\text { observed on the secondary vessel } \\
\text { wall were caused by the inleakage of } \\
\text { water. }\end{array}$ \\
\hline
\end{tabular}




\begin{tabular}{|c|c|c|c|c|c|c|c|c|}
\hline \multirow{2}{*}{$\frac{\text { AREA }}{\mathrm{H}}$} & \multirow{2}{*}{$\begin{array}{c}\text { TANK OR } \\
\text { ANCILLARY } \\
29\end{array}$} & \multicolumn{2}{|c|}{$\frac{\text { ACCESS OPENING }}{(A \text { OR I) }}$} & \multirow{2}{*}{$\begin{array}{c}\text { DATE } \\
03 / 07 / 06\end{array}$} & \multicolumn{3}{|c|}{$\begin{array}{l}\text { INSPECTION METHOD } \\
\text { IDENTIFICATION NUMBER }\end{array}$} & \multirow{2}{*}{$\begin{array}{l}\text { REMARKS } \\
\text { Remote visual tank wall inspection } \\
\text { revealed no areas of concern since } \\
\text { last evaluated on } 1 / 7 / 05 \text {. An } \\
\text { increase in stains and marks } \\
\text { observed on the secondary vessel } \\
\text { wall were caused by the inleakage of } \\
\text { water. }\end{array}$} \\
\hline & & A-03 & (A) & & WAP & I & P06040:11 & \\
\hline $\mathrm{H}$ & 29 & A-04 & (A) & 03/07/06 & WAP & I & P06040:09 & $\begin{array}{l}\text { Remote visual tank wall inspection } \\
\text { revealed no areas of concern since } \\
\text { last evaluated on } 1 / 7 / 05 \text {. }\end{array}$ \\
\hline $\mathrm{H}$ & 29 & A-04 & (A) & 09/22/06 & CCTV & I & 1006 & $\begin{array}{l}\text { The magnetically mounted tank wall } \\
\text { thermocouple was properly } \\
\text { positioned per T-DS-G-00010. }\end{array}$ \\
\hline $\mathrm{H}$ & 29 & P-01 & (A) & 03/07/06 & WAP & l & P06040:07 & $\begin{array}{l}\text { Remote visual tank wall inspection } \\
\text { revealed no areas of concern since } \\
\text { last evaluated on } 1 / 7 / 05 \text {. }\end{array}$ \\
\hline $\mathrm{H}$ & 29 & P-02 & (A) & 03/07/06 & WAP & I & P06040:08 & $\begin{array}{l}\text { Remote visual tank wall inspection } \\
\text { revealed no areas of concern since } \\
\text { last evaluated on } 2 / 5 / 05 \text {. }\end{array}$ \\
\hline $\mathrm{H}$ & 29 & P-02 & (A) & $05 / 31 / 06$ & UT & I & UT-06-002 & $\begin{array}{l}\text { UT scanning was performed on a } \\
\text { vertical strip } 8.5 \text { inches wide for the } \\
\text { entire accessible height of the } \\
\text { primary vessel wall. Analysis of the } \\
\text { data revealed no reportable cracking, } \\
\text { thinning or pitting in the primary wall. } \\
\text { This information is included in } \\
\text { document WSRC-TR-2006-00002. }\end{array}$ \\
\hline $\mathrm{H}$ & 29 & P-03 & (A) & $07 / 11 / 06$ & $\mathrm{DP}$ & I & P06198:01-27 & $\begin{array}{l}\text { Remote visual tank wall inspection } \\
\text { revealed no areas of concern since } \\
\text { last evaluated on } 1 / 07 / 05\end{array}$ \\
\hline $\mathrm{H}$ & 29 & P-04 & (A) & 03/07/06 & WAP & I & P06040:04 & $\begin{array}{l}\text { Remote visual tank wall inspection } \\
\text { revealed no areas of concern since } \\
\text { last evaluated on } 1 / 7 / 05 \text {. }\end{array}$ \\
\hline $\mathrm{H}$ & 29 & P-05 & (A) & 03/07/06 & WAP & I & P06040:05 & $\begin{array}{l}\text { Remote visual tank wall inspection } \\
\text { revealed no areas of concern since } \\
\text { last evaluated on } 2 / 5 / 05 \text {. }\end{array}$ \\
\hline
\end{tabular}




\begin{tabular}{|c|c|c|c|c|c|c|c|}
\hline$\frac{\text { AREA }}{\mathrm{H}}$ & $\begin{array}{c}\text { TANK OR } \\
\text { ANCILLARY } \\
29\end{array}$ & $\frac{\text { ACCESS OPENING }}{\text { (A OR I) }}$ & $\begin{array}{c}\text { DATE } \\
05 / 23 / 06\end{array}$ & \multicolumn{3}{|c|}{$\begin{array}{l}\text { INSPECTION METHOD } \\
\text { IDENTIFICATION NUMBER }\end{array}$} & \multirow[b]{2}{*}{$\begin{array}{l}\text { REMARKS } \\
\text { UT scanning was performed on a } \\
\text { vertical strip } 8.5 \text { inches wide for the } \\
\text { entire accessible height of the } \\
\text { primary vessel wall. A two square } \\
\text { foot area was also scanned on all } \\
\text { four of the secondary liner plates. } \\
\text { Three } 10 \text { inch wide areas were also } \\
\text { scanned on the annulus floor. } \\
\text { Analysis of the data revealed no } \\
\text { reportable cracking, thinning or } \\
\text { pitting in the primary wall. Reportable } \\
\text { thickness was detected in secondary } \\
\text { wall plate } 1 . \text { This information is } \\
\text { included in document WSRC-TR } \\
\text {-2006-00002. }\end{array}$} \\
\hline $\mathrm{H}$ & 29 & P-05 (A) & $05 / 23 / 06$ & UT & I & UT-06-001 & \\
\hline
\end{tabular}

$\mathrm{H}$

29

P-06 (A)

$\mathrm{H}$

$\mathrm{H}$

$\mathrm{H}$

29

P-09 (A)

P-07 (A)

P-08 (A)
$\mathrm{H}$

29

29

P-10 (A)

07/11/06

29

P-09 (A)

07/11/06

$\mathrm{H}$

03/07/06
07/11/06

03/07/06

06/07/06

DP I P06199:01-27

WAP I P06040:01

WAP I P06040:02

UT I UT-06-003

DP I P06200:01-27

DP I P06202:01-27
Remote visual tank wall inspection revealed no areas of concern since last evaluated on $1 / 07 / 05$. The annulus jet beneath the A-01 riser showed no degradation per T-DS-G -00043 .

Remote visual tank wall inspection revealed no areas of concern since last evaluated on 1/7/05.

Remote visual tank wall inspection revealed no areas of concern since last evaluated on $2 / 5 / 05$. Stains and marks on the secondary vessel wall were caused by the inleakage of water.

UT scanning was performed on a vertical strip 8.5 inches wide for the primary vessel wall. Analysis of the data revealed no reportable cracking, thinning or pitting in the primary wall. This information is included in document WSRC-TR-2006-00002.

Remote visual tank wall inspection revealed no areas of concern since last evaluated on 1/07/05.

Remote visual tank wall inspection revealed no areas of concern since last evaluated on 1/07/05.

Remote visual tank wall inspection revealed no areas of concern since last evaluated on 2/5/05. entire accessible height of the

P-11 (A) 


\begin{tabular}{|c|c|c|c|c|c|c|}
\hline AREA & $\begin{array}{l}\text { TANK OR } \\
\text { ANCILLARY }\end{array}$ & $\frac{\text { ACCESS OPENING }}{(A \text { OR I) }}$ & DATE & \multicolumn{3}{|c|}{$\begin{array}{l}\text { INSPECTION METHOD } \\
\text { IDENTIFICATION NUMBER }\end{array}$} \\
\hline $\mathrm{H}$ & 29 & $\mathrm{P}-12 \quad(\mathrm{~A})$ & 03/07/06 & WAP & I & P06040:10 \\
\hline $\mathrm{H}$ & 29 & $\mathrm{P}-12 \quad(\mathrm{~A})$ & $06 / 14 / 06$ & UT & I & UT-06-004 \\
\hline $\mathrm{H}$ & 29 & $\mathrm{P}-13 \quad(\mathrm{~A})$ & $07 / 11 / 06$ & $\mathrm{DP}$ & I & P06201:01-27 \\
\hline $\mathrm{H}$ & 29 & $\mathrm{P}-14 \quad(\mathrm{~A})$ & 03/07/06 & WAP & I & P06040:12 \\
\hline $\mathrm{H}$ & 29 & $\mathrm{P}-14 \quad(\mathrm{~A})$ & $06 / 29 / 06$ & UT & 1 & UT-06-005 \\
\hline
\end{tabular}




\begin{tabular}{|c|c|c|c|c|c|c|c|}
\hline \multirow{2}{*}{$\begin{array}{l}\text { AREA } \\
\mathrm{H}\end{array}$} & \multirow{2}{*}{$\begin{array}{c}\begin{array}{c}\text { TANK OR } \\
\text { ANCILLARY }\end{array} \\
29 / 32\end{array}$} & $\frac{\text { ACCESS OPENING }}{\text { (A OR I) }}$ & \multirow{2}{*}{$\begin{array}{l}\text { DATE } \\
06 / 26 / 06\end{array}$} & \multicolumn{3}{|c|}{$\begin{array}{l}\text { INSPECTION METHOD } \\
\text { IDENTIFICATION NUMBER }\end{array}$} & \multirow{2}{*}{$\begin{array}{l}\text { REMARKS } \\
\text { Inspection of the transfer lines within } \\
\text { the clean out port revealed no } \\
\text { degradation per T-DS-G-00001. }\end{array}$} \\
\hline & & COP \#112 & & DSP & l & P06166:01-06 & \\
\hline $\mathrm{H}$ & 30 & $A-01 \quad(A)$ & 03/07/06 & WAP & I & P06041:06 & $\begin{array}{l}\text { Remote visual tank wall inspection } \\
\text { revealed no areas of concern since } \\
\text { last evaluated on } 1 / 7 / 05 \text {. The } \\
\text { annulus jet showed no degradation } \\
\text { per T-DS-G-00043. }\end{array}$ \\
\hline $\mathrm{H}$ & 30 & $\mathrm{~A}-02 \quad(\mathrm{~A})$ & 03/07/06 & WAP & I & P06041:09 & $\begin{array}{l}\text { Remote visual tank wall inspection } \\
\text { revealed no areas of concern since } \\
\text { last evaluated on } 1 / 7 / 05 \text {. }\end{array}$ \\
\hline $\mathrm{H}$ & 30 & $\mathrm{~A}-03 \quad(\mathrm{~A})$ & 03/07/06 & WAP & I & P06041:12 & $\begin{array}{l}\text { Remote visual tank wall inspection } \\
\text { revealed no areas of concern since } \\
\text { last evaluated on } 1 / 7 / 05 .\end{array}$ \\
\hline $\mathrm{H}$ & 30 & $\mathrm{~A}-04 \quad(\mathrm{~A})$ & 03/07/06 & WAP & l & P06041:03 & $\begin{array}{l}\text { Remote visual tank wall inspection } \\
\text { revealed no areas of concern since } \\
\text { last evaluated on } 1 / 7 / 05 \text {. }\end{array}$ \\
\hline $\mathrm{H}$ & 30 & $\mathrm{~A}-04 \quad(\mathrm{~A})$ & $09 / 22 / 06$ & CCTV & I & 1002 & $\begin{array}{l}\text { The magnetically mounted tank wall } \\
\text { thermocouple was properly } \\
\text { positioned per T-DS-G-00010. }\end{array}$ \\
\hline $\mathrm{H}$ & 30 & $\mathrm{P}-01 \quad(\mathrm{~A})$ & 03/07/06 & WAP & l & P06041:01 & $\begin{array}{l}\text { Remote visual tank wall inspection } \\
\text { revealed no areas of concern since } \\
\text { last evaluated on } 1 / 7 / 05 \text {. }\end{array}$ \\
\hline $\mathrm{H}$ & 30 & $\mathrm{P}-02 \quad(\mathrm{~A})$ & 03/07/06 & WAP & I & P06041:02 & $\begin{array}{l}\text { Remote visual tank wall inspection } \\
\text { revealed no areas of concern since } \\
\text { last evaluated on } 2 / 5 / 05 \text {. }\end{array}$ \\
\hline $\mathrm{H}$ & 30 & $\mathrm{P}-03 \quad(\mathrm{~A})$ & $07 / 11 / 06$ & DP & l & P06203:01-27 & $\begin{array}{l}\text { Remote visual tank wall inspection } \\
\text { revealed no areas of concern since } \\
\text { last evaluated on } 1 / 07 / 05 \text {. }\end{array}$ \\
\hline $\mathrm{H}$ & 30 & $\mathrm{P}-04 \quad(\mathrm{~A})$ & 03/07/06 & WAP & I & P06041:04 & $\begin{array}{l}\text { Remote visual tank wall inspection } \\
\text { revealed no areas of concern since } \\
\text { last evaluated on } 1 / 7 / 05 \text {. }\end{array}$ \\
\hline $\mathrm{H}$ & 30 & $\mathrm{P}-05 \quad(\mathrm{~A})$ & 03/07/06 & WAP & I & P06041:05 & $\begin{array}{l}\text { Remote visual tank wall inspection } \\
\text { revealed no areas of concern since } \\
\text { last evaluated on } 2 / 5 / 05 \text {. }\end{array}$ \\
\hline $\mathrm{H}$ & 30 & $\mathrm{P}-06 \quad(\mathrm{~A})$ & 07/11/06 & DP & I & P06204:01-28 & $\begin{array}{l}\text { Remote visual tank wall inspection } \\
\text { revealed no areas of concern since } \\
\text { last evaluated on } 1 / 07 / 05 \text {. The } \\
\text { annulus jet beneath the A- } 01 \text { riser } \\
\text { showed no degradation per T-DS-G } \\
-00043 \text {. }\end{array}$ \\
\hline
\end{tabular}




\begin{tabular}{|c|c|c|c|c|c|c|c|c|}
\hline \multirow{2}{*}{ AREA } & \multirow{2}{*}{$\begin{array}{c}\begin{array}{c}\text { TANK OR } \\
\text { ANCILLARY }\end{array} \\
30\end{array}$} & \multicolumn{2}{|c|}{$\begin{array}{c}\text { ACCESS OPENING } \\
\text { (A OR I) }\end{array}$} & \multirow{2}{*}{$\begin{array}{c}\text { DATE } \\
03 / 07 / 06\end{array}$} & \multicolumn{3}{|c|}{$\begin{array}{l}\text { INSPECTION METHOD } \\
\text { IDENTIFICATION NUMBER }\end{array}$} & \multirow{2}{*}{$\begin{array}{l}\text { REMARKS } \\
\text { Remote visual tank wall inspection } \\
\text { revealed no areas of concern since } \\
\text { last evaluated on } 1 / 7 / 05 \text {. }\end{array}$} \\
\hline & & P-07 & (A) & & WAP & I & P06041:07 & \\
\hline $\mathrm{H}$ & 30 & P-08 & (A) & 03/07/06 & WAP & I & P06041:08 & $\begin{array}{l}\text { Remote visual tank wall inspection } \\
\text { revealed no areas of concern since } \\
\text { last evaluated on } 2 / 5 / 05 \text {. }\end{array}$ \\
\hline $\mathrm{H}$ & 30 & P-09 & (A) & 07/11/06 & DP & I & P06205:01-28 & $\begin{array}{l}\text { Remote visual tank wall inspection } \\
\text { revealed no areas of concern since } \\
\text { last evaluated on } 1 / 07 / 05 \text {. }\end{array}$ \\
\hline $\mathrm{H}$ & 30 & P-10 & (A) & 07/11/06 & DP & I & P06206:01-28 & $\begin{array}{l}\text { Remote visual tank wall inspection } \\
\text { revealed no areas of concern since } \\
\text { last evaluated on } 1 / 07 / 05 \text {. }\end{array}$ \\
\hline $\mathrm{H}$ & 30 & P-11 & (A) & 03/07/06 & WAP & I & P06041:10 & $\begin{array}{l}\text { Remote visual tank wall inspection } \\
\text { revealed no areas of concern since } \\
\text { last evaluated on } 1 / 7 / 05 \text {. }\end{array}$ \\
\hline $\mathrm{H}$ & 30 & P-12 & (A) & 03/07/06 & WAP & I & P06041:11 & $\begin{array}{l}\text { Remote visual tank wall inspection } \\
\text { revealed no areas of concern since } \\
\text { last evaluated on } 1 / 7 / 05 \text {. }\end{array}$ \\
\hline $\mathrm{H}$ & 30 & $P-13$ & (A) & 07/11/06 & DP & l & P06207:01-28 & $\begin{array}{l}\text { Remote visual tank wall inspection } \\
\text { revealed no areas of concern since } \\
\text { last evaluated on } 1 / 07 / 05 \text {. Stains } \\
\text { observed on the secondary vessel } \\
\text { wall were caused by the inleakage of } \\
\text { water. }\end{array}$ \\
\hline $\mathrm{H}$ & 30 & P-14 & (A) & 03/07/06 & WAP & I & P06041:13 & $\begin{array}{l}\text { Remote visual tank wall inspection } \\
\text { revealed no areas of concern since } \\
\text { last evaluated on } 2 / 5 / 05 \text {. }\end{array}$ \\
\hline $\mathrm{H}$ & 31 & COP \#102 & & 06/26/06 & DSP & I & P06157:01-06 & $\begin{array}{l}\text { Inspection of the transfer lines within } \\
\text { the clean out port revealed no } \\
\text { degradation per T-DS-G- } 00001 \text {. }\end{array}$ \\
\hline $\mathrm{H}$ & 31 & $A-01$ & (A) & $03 / 13 / 06$ & WAP & I & P06048:01 & $\begin{array}{l}\text { Remote visual tank wall inspection } \\
\text { revealed no areas of concern since } \\
\text { last evaluated on } 1 / 7 / 05 \text {. The } \\
\text { annulus jet showed no degradation } \\
\text { per T-DS-G-00043. }\end{array}$ \\
\hline $\mathrm{H}$ & 31 & A-02 & (A) & $03 / 13 / 06$ & WAP & I & P06048:03 & $\begin{array}{l}\text { Remote visual tank wall inspection } \\
\text { revealed no areas of concern since } \\
\text { last evaluated on } 1 / 7 / 05 \text {. }\end{array}$ \\
\hline $\mathrm{H}$ & 31 & A-03 & (A) & 03/13/06 & WAP & I & P06048:07 & $\begin{array}{l}\text { Remote visual tank wall inspection } \\
\text { revealed no areas of concern since } \\
\text { last evaluated on } 1 / 7 / 05 \text {. }\end{array}$ \\
\hline
\end{tabular}




\begin{tabular}{|c|c|c|c|c|c|c|c|c|}
\hline \multirow{2}{*}{ AREA } & \multirow{2}{*}{$\begin{array}{c}\begin{array}{c}\text { TANK OR } \\
\text { ANCILLARY }\end{array} \\
31\end{array}$} & \multicolumn{2}{|c|}{$\frac{\text { ACCESS OPENING }}{\text { (A OR I) }}$} & \multirow{2}{*}{$\frac{\text { DATE }}{03 / 13 / 06}$} & \multicolumn{3}{|c|}{$\begin{array}{l}\text { INSPECTION METHOD } \\
\text { IDENTIFICATION NUMBER }\end{array}$} & \multirow{2}{*}{$\begin{array}{l}\qquad \text { REMARKS } \\
\text { Remote visual tank wall inspection } \\
\text { revealed no areas of concern since } \\
\text { last evaluated on } 1 / 7 / 05 \text {. }\end{array}$} \\
\hline & & A-04 & (A) & & WAP & l & P06048:04 & \\
\hline $\mathrm{H}$ & 31 & A-04 & (A) & $09 / 22 / 06$ & CCTV & I & 1344 & $\begin{array}{l}\text { The magnetically mounted tank wall } \\
\text { thermocouple was properly } \\
\text { positioned per T-DS-G-00010. }\end{array}$ \\
\hline $\mathrm{H}$ & 31 & P-01 & (A) & 03/13/06 & WAP & I & P06048:08 & $\begin{array}{l}\text { Remote visual tank wall inspection } \\
\text { revealed no areas of concern since } \\
\text { last evaluated on } 1 / 7 / 05 \text {. }\end{array}$ \\
\hline $\mathrm{H}$ & 31 & P-02 & (A) & 03/13/06 & WAP & I & P06048:09 & $\begin{array}{l}\text { Remote visual tank wall inspection } \\
\text { revealed no areas of concern since } \\
\text { last evaluated on } 1 / 18 / 05 \text {. }\end{array}$ \\
\hline $\mathrm{H}$ & 31 & P-03 & (A) & 07/11/06 & DP & I & P06208:01-27 & $\begin{array}{l}\text { Remote visual tank wall inspection } \\
\text { revealed no areas of concern since } \\
\text { last evaluated on } 1 / 07 / 05 \text {. }\end{array}$ \\
\hline $\mathrm{H}$ & 31 & P-04 & (A) & 03/13/06 & WAP & I & P06048:05 & $\begin{array}{l}\text { Remote visual tank wall inspection } \\
\text { revealed no areas of concern since } \\
\text { last evaluated on } 1 / 7 / 05 \text {. }\end{array}$ \\
\hline $\mathrm{H}$ & 31 & P-05 & (A) & 03/13/06 & WAP & l & P06048:06 & $\begin{array}{l}\text { Remote visual tank wall inspection } \\
\text { revealed no areas of concern since } \\
\text { last evaluated on } 1 / 18 / 05 \text {. }\end{array}$ \\
\hline $\mathrm{H}$ & 31 & P-06 & (A) & 07/11/06 & DP & I & P06209:01-27 & $\begin{array}{l}\text { Remote visual tank wall inspection } \\
\text { revealed no areas of concern since } \\
\text { last evaluated on } 1 / 07 / 05 \text {. The } \\
\text { annulus jet beneath the A-01 riser } \\
\text { showed no degradation per T-DS-G } \\
-00043 \text {. }\end{array}$ \\
\hline $\mathrm{H}$ & 31 & P-07 & (A) & 03/13/06 & WAP & I & P06048:02 & $\begin{array}{l}\text { Remote visual tank wall inspection } \\
\text { revealed no areas of concern since } \\
\text { last evaluated on } 1 / 7 / 05 \text {. }\end{array}$ \\
\hline $\mathrm{H}$ & 31 & P-08 & (A) & 03/03/06 & WAP & I & P06047:01 & $\begin{array}{l}\text { Remote visual tank wall inspection } \\
\text { revealed no areas of concern since } \\
\text { last evaluated on } 1 / 18 / 05 \text {. }\end{array}$ \\
\hline $\mathrm{H}$ & 31 & P-09 & (A) & $07 / 11 / 06$ & DP & I & P06210:01-28 & $\begin{array}{l}\text { Remote visual tank wall inspection } \\
\text { revealed no areas of concern since } \\
\text { last evaluated on } 1 / 07 / 05 \text {. }\end{array}$ \\
\hline $\mathrm{H}$ & 31 & $P-10$ & (A) & $07 / 11 / 06$ & DP & I & P06211:01-25 & $\begin{array}{l}\text { Remote visual tank wall inspection } \\
\text { revealed no areas of concern since } \\
\text { last evaluated on } 1 / 07 / 05 \text {. }\end{array}$ \\
\hline $\mathrm{H}$ & 31 & $\mathrm{P}-11$ & (A) & 03/03/06 & WAP & l & P06047:02 & $\begin{array}{l}\text { Remote visual tank wall inspection } \\
\text { revealed no areas of concern since } \\
\text { last evaluated on } 1 / 7 / 05 \text {. }\end{array}$ \\
\hline
\end{tabular}




\begin{tabular}{|c|c|c|c|c|c|c|c|c|}
\hline \multirow{2}{*}{ AREA } & \multirow{2}{*}{$\begin{array}{c}\begin{array}{c}\text { TANK OR } \\
\text { ANCILLARY }\end{array} \\
31\end{array}$} & \multicolumn{2}{|c|}{$\frac{\text { ACCESS OPENING }}{(A \text { OR I) }}$} & \multirow{2}{*}{$\begin{array}{l}\underline{\text { DATE }} \\
03 / 03 / 06\end{array}$} & \multicolumn{3}{|c|}{$\begin{array}{l}\text { INSPECTION METHOD } \\
\text { IDENTIFICATION NUMBER }\end{array}$} & \multirow{2}{*}{$\begin{array}{l}\text { REMARKS } \\
\text { Remote visual tank wall inspection } \\
\text { revealed no areas of concern since } \\
\text { last evaluated on } 1 / 18 / 05 \text {. }\end{array}$} \\
\hline & & P-12 & (A) & & WAP & l & P06047:03 & \\
\hline $\mathrm{H}$ & 31 & P-13 & (A) & $07 / 11 / 06$ & DP & I & P06212:01-27 & $\begin{array}{l}\text { Remote visual tank wall inspection } \\
\text { revealed no areas of concern since } \\
\text { last evaluated on } 1 / 07 / 05 \text {. }\end{array}$ \\
\hline $\mathrm{H}$ & 31 & P-14 & (A) & 03/03/06 & WAP & l & P06047:04 & $\begin{array}{l}\text { Remote visual tank wall inspection } \\
\text { revealed no areas of concern since } \\
\text { last evaluated on } 1 / 18 / 05 \text {. }\end{array}$ \\
\hline $\mathrm{H}$ & 32 & COP \#109 & & 06/26/06 & DSP & l & P06163:01-04 & $\begin{array}{l}\text { Inspection of the transfer lines within } \\
\text { the clean out port revealed no } \\
\text { degradation per T-DS-G-00001. }\end{array}$ \\
\hline $\mathrm{H}$ & 32 & COP \#110 & & 06/26/06 & DSP & I & P06164:01-06 & $\begin{array}{l}\text { Inspection of the transfer lines within } \\
\text { the clean out port revealed no } \\
\text { degradation per T-DS-G-00001. }\end{array}$ \\
\hline $\mathrm{H}$ & 32 & COP \#111 & & 06/26/06 & DSP & I & P06165:01-06 & $\begin{array}{l}\text { Inspection of the transfer lines within } \\
\text { the clean out port revealed no } \\
\text { degradation per T-DS-G- } 00001 \text {. }\end{array}$ \\
\hline $\mathrm{H}$ & 32 & A-01 & (A) & 03/13/06 & WAP & l & P06036:01 & $\begin{array}{l}\text { Remote visual tank wall inspection } \\
\text { revealed no areas of concern since } \\
\text { last evaluated on } 1 / 8 / 05 \text {. }\end{array}$ \\
\hline $\mathrm{H}$ & 32 & A-02 & (A) & 03/13/06 & WAP & I & P06036:06 & $\begin{array}{l}\text { Remote visual tank wall inspection } \\
\text { revealed no areas of concern since } \\
\text { last evaluated on } 1 / 8 / 05 \text {. }\end{array}$ \\
\hline $\mathrm{H}$ & 32 & A-03 & (A) & 03/13/06 & WAP & I & P06036:10 & $\begin{array}{l}\text { Remote visual tank wall inspection } \\
\text { revealed no areas of concern since } \\
\text { last evaluated on } 1 / 8 / 05 \text {. }\end{array}$ \\
\hline $\mathrm{H}$ & 32 & A-04 & (A) & 03/13/06 & WAP & I & P06036:12 & $\begin{array}{l}\text { Remote visual tank wall inspection } \\
\text { revealed no areas of concern since } \\
\text { last evaluated on } 1 / 8 / 05 \text {. }\end{array}$ \\
\hline $\mathrm{H}$ & 32 & A-04 & (A) & 09/22/06 & CCTV & I & 1462 & $\begin{array}{l}\text { The magnetically mounted tank wall } \\
\text { thermocouple was properly } \\
\text { positioned per T-DS-G-00010. }\end{array}$ \\
\hline $\mathrm{H}$ & 32 & P-01 & (A) & 03/13/06 & WAP & I & P06036:04 & $\begin{array}{l}\text { Remote visual tank wall inspection } \\
\text { revealed no areas of concern since } \\
\text { last evaluated on } 1 / 8 / 05 \text {. }\end{array}$ \\
\hline $\mathrm{H}$ & 32 & P-02 & (A) & $05 / 11 / 06$ & WAP & I & P06086:02 & $\begin{array}{l}\text { Remote visual tank wall inspection } \\
\text { revealed no areas of concern since } \\
\text { last evaluated on } 2 / 7 / 05 \text {. }\end{array}$ \\
\hline $\mathrm{H}$ & 32 & P-03 & (A) & $07 / 11 / 06$ & DP & I & P06191:01-27 & $\begin{array}{l}\text { Remote visual tank wall inspection } \\
\text { revealed no areas of concern since } \\
\text { last evaluated on } 1 / 08 / 05 \text {. }\end{array}$ \\
\hline
\end{tabular}




\begin{tabular}{|c|c|c|c|c|c|c|c|c|}
\hline \multirow{2}{*}{ AREA } & \multirow{2}{*}{ 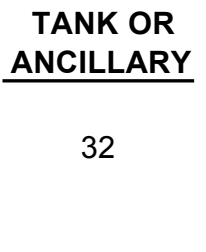 } & \multicolumn{2}{|c|}{$\frac{\text { ACCESS OPENING }}{(A \text { OR I) }}$} & \multirow{2}{*}{$\begin{array}{l}\text { DATE } \\
03 / 13 / 06\end{array}$} & \multicolumn{3}{|c|}{$\begin{array}{l}\text { INSPECTION METHOD } \\
\text { IDENTIFICATION NUMBER }\end{array}$} & \multirow{2}{*}{$\begin{array}{l}\text { REMARKS } \\
\text { Remote visual tank wall inspection } \\
\text { revealed no areas of concern since } \\
\text { last evaluated on } 1 / 8 / 05 \text {. }\end{array}$} \\
\hline & & P-04 & (A) & & WAP & l & P06036:13 & \\
\hline $\mathrm{H}$ & 32 & P-05 & (A) & 05/11/06 & WAP & I & P06086:03 & $\begin{array}{l}\text { Remote visual tank wall inspection } \\
\text { revealed no areas of concern since } \\
\text { last evaluated on } 2 / 5 / 05 \text {. }\end{array}$ \\
\hline $\mathrm{H}$ & 32 & P-06 & (A) & 07/11/06 & $\mathrm{DP}$ & I & P06192:01-27 & $\begin{array}{l}\text { Remote visual tank wall inspection } \\
\text { revealed no areas of concern since } \\
\text { last evaluated on } 1 / 08 / 05 \text {. The } \\
\text { annulus jet beneath the A- } 01 \text { riser } \\
\text { showed no degradation per T-DS-G } \\
\text {-00043. }\end{array}$ \\
\hline $\mathrm{H}$ & 32 & P-07 & (A) & 03/13/06 & WAP & l & P06036:02 & $\begin{array}{l}\text { Remote visual tank wall inspection } \\
\text { revealed no areas of concern since } \\
\text { last evaluated on } 1 / 8 / 05 \text {. }\end{array}$ \\
\hline $\mathrm{H}$ & 32 & P-08 & (A) & 03/13/06 & WAP & I & P06036:03 & $\begin{array}{l}\text { Remote visual tank wall inspection } \\
\text { revealed no areas of concern since } \\
\text { last evaluated on } 2 / 5 / 05 \text {. Stains on } \\
\text { the secondary vessel wall were } \\
\text { caused by the inleakage of water. }\end{array}$ \\
\hline $\mathrm{H}$ & 32 & P-09 & (A) & $07 / 11 / 06$ & $\mathrm{DP}$ & l & P06193:01-27 & $\begin{array}{l}\text { Remote visual tank wall inspection } \\
\text { revealed no areas of concern since } \\
\text { last evaluated on } 1 / 08 / 05 \text {. }\end{array}$ \\
\hline $\mathrm{H}$ & 32 & P-10 & (A) & 07/11/06 & DP & I & P06194:01-27 & $\begin{array}{l}\text { Remote visual tank wall inspection } \\
\text { revealed no areas of concern since } \\
\text { last evaluated on } 1 / 08 / 05 \text {. }\end{array}$ \\
\hline $\mathrm{H}$ & 32 & $\mathrm{P}-11$ & (A) & $05 / 11 / 06$ & WAP & I & P06086:04 & $\begin{array}{l}\text { Remote visual tank wall inspection } \\
\text { revealed no areas of concern since } \\
\text { last evaluated on } 1 / 8 / 05 \text {. Changes in } \\
\text { the stains on top of the ventilation } \\
\text { duct were caused by the inleakage of } \\
\text { water. }\end{array}$ \\
\hline $\mathrm{H}$ & 32 & P-12 & (A) & $05 / 11 / 06$ & WAP & I & P06086:01 & $\begin{array}{l}\text { Remote visual tank wall inspection } \\
\text { revealed no areas of concern since } \\
\text { last evaluated on } 2 / 7 / 05 \text {. }\end{array}$ \\
\hline $\mathrm{H}$ & 32 & P-13 & (A) & 07/12/06 & DP & I & P06224:01-28 & $\begin{array}{l}\text { Remote visual tank wall inspection } \\
\text { revealed no areas of concern since } \\
\text { last evaluated on } 1 / 08 / 05 \text {. }\end{array}$ \\
\hline $\mathrm{H}$ & 32 & $\mathrm{P}-14$ & (A) & 03/13/06 & WAP & I & P06036:08 & $\begin{array}{l}\text { Remote visual tank wall inspection } \\
\text { revealed no areas of concern since } \\
\text { last evaluated on } 2 / 7 / 05 \text {. }\end{array}$ \\
\hline $\mathrm{H}$ & 32 & P-15 & (A) & 03/13/06 & WAP & I & P06036:09 & $\begin{array}{l}\text { Remote visual tank wall inspection } \\
\text { revealed no areas of concern since } \\
\text { last evaluated on } 2 / 7 / 05 \text {. }\end{array}$ \\
\hline
\end{tabular}




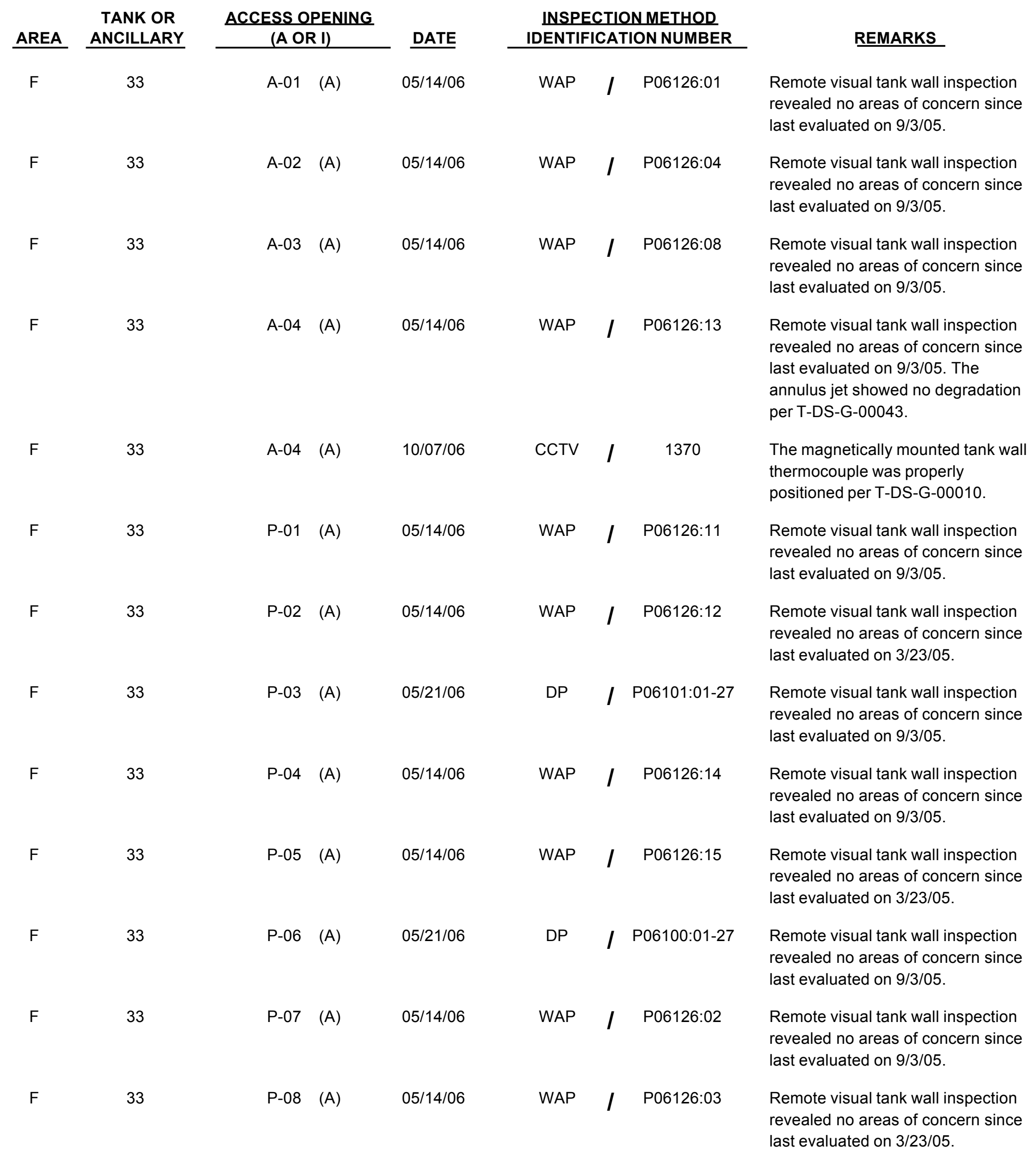




\begin{tabular}{|c|c|c|c|c|c|c|c|c|}
\hline \multirow{2}{*}{ AREA } & \multirow{2}{*}{$\begin{array}{c}\begin{array}{c}\text { TANK OR } \\
\text { ANCILLARY }\end{array} \\
33\end{array}$} & \multicolumn{2}{|c|}{$\frac{\text { ACCESS OPENING }}{(A \text { OR I) }}$} & \multirow{2}{*}{$\begin{array}{l}\text { DATE } \\
05 / 21 / 06\end{array}$} & \multicolumn{3}{|c|}{$\begin{array}{l}\text { INSPECTION METHOD } \\
\text { IDENTIFICATION NUMBER }\end{array}$} & \multirow{2}{*}{$\begin{array}{l}\text { REMARKS } \\
\text { Remote visual tank wall inspection } \\
\text { revealed no areas of concern since } \\
\text { last evaluated on } 9 / 3 / 05 \text {. }\end{array}$} \\
\hline & & P-09 & (A) & & DP & l & P06102:01-27 & \\
\hline $\mathrm{F}$ & 33 & $P-10$ & (A) & $05 / 21 / 06$ & DP & l & P06103:01-29 & $\begin{array}{l}\text { Remote visual tank wall inspection } \\
\text { revealed no areas of concern since } \\
\text { last evaluated on } 9 / 3 / 05 \text {. }\end{array}$ \\
\hline $\mathrm{F}$ & 33 & $P-11$ & (A) & $05 / 14 / 06$ & WAP & l & P06126:05 & $\begin{array}{l}\text { Remote visual tank wall inspection } \\
\text { revealed no areas of concern since } \\
\text { last evaluated on } 3 / 23 / 05 \text {. }\end{array}$ \\
\hline $\mathrm{F}$ & 33 & $\mathrm{P}-12$ & (A) & 05/14/06 & WAP & l & P06126:06 & $\begin{array}{l}\text { Remote visual tank wall inspection } \\
\text { revealed no areas of concern since } \\
\text { last evaluated on } 3 / 23 / 05 \text {. }\end{array}$ \\
\hline $\mathrm{F}$ & 33 & $P-13$ & (A) & 05/21/06 & DP & l & P06104:01-27 & $\begin{array}{l}\text { Remote visual tank wall inspection } \\
\text { revealed no areas of concern since } \\
\text { last evaluated on } 9 / 3 / 05 \text {. }\end{array}$ \\
\hline$F$ & 33 & P-14 & (A) & 05/14/06 & WAP & l & P06126:07 & $\begin{array}{l}\text { Remote visual tank wall inspection } \\
\text { revealed no areas of concern since } \\
\text { last evaluated on } 3 / 23 / 05 \text {. }\end{array}$ \\
\hline $\mathrm{F}$ & 33 & P-15 & (A) & $05 / 14 / 06$ & WAP & l & P06126:09 & $\begin{array}{l}\text { Remote visual tank wall inspection } \\
\text { revealed no areas of concern since } \\
\text { last evaluated on } 3 / 23 / 05 \text {. }\end{array}$ \\
\hline $\mathrm{F}$ & 33 & $P-16$ & (A) & $05 / 14 / 06$ & WAP & l & P06126:10 & $\begin{array}{l}\text { Remote visual tank wall inspection } \\
\text { revealed no areas of concern since } \\
\text { last evaluated on } 9 / 3 / 05 \text {. }\end{array}$ \\
\hline $\mathrm{F}$ & 34 & A-01 & (A) & 05/20/06 & WAP & l & P06125:06 & $\begin{array}{l}\text { Remote visual tank wall inspection } \\
\text { revealed no areas of concern since } \\
\text { last evaluated on } 9 / 3 / 05 \text {. }\end{array}$ \\
\hline $\mathrm{F}$ & 34 & A-02 & (A) & 05/20/06 & WAP & l & P06125:13 & $\begin{array}{l}\text { Remote visual tank wall inspection } \\
\text { revealed no areas of concern since } \\
\text { last evaluated on } 9 / 3 / 05 \text {. }\end{array}$ \\
\hline $\mathrm{F}$ & 34 & A-03 & (A) & 05/20/06 & WAP & l & P06125:10 & $\begin{array}{l}\text { Remote visual tank wall inspection } \\
\text { revealed no areas of concern since } \\
\text { last evaluated on } 9 / 3 / 05 \text {. }\end{array}$ \\
\hline $\mathrm{F}$ & 34 & A-04 & (A) & 05/20/06 & WAP & l & P06125:03 & $\begin{array}{l}\text { Remote visual tank wall inspection } \\
\text { revealed no areas of concern since } \\
\text { last evaluated on } 9 / 3 / 05 \text {. The } \\
\text { annulus jet showed no degradation } \\
\text { per T-DS-G-00043. }\end{array}$ \\
\hline $\mathrm{F}$ & 34 & A-04 & (A) & $11 / 02 / 06$ & CCTV & l & 1499 & $\begin{array}{l}\text { The magnetically mounted tank wall } \\
\text { thermocouple was properly } \\
\text { positioned per T-DS-G-00010. }\end{array}$ \\
\hline
\end{tabular}




\begin{tabular}{|c|c|c|c|c|c|c|c|c|}
\hline \multirow{2}{*}{ AREA } & \multirow{2}{*}{$\begin{array}{c}\begin{array}{c}\text { TANK OR } \\
\text { ANCILLARY }\end{array} \\
34\end{array}$} & \multicolumn{2}{|c|}{$\frac{\text { ACCESS OPENING }}{(A \text { OR I) }}$} & \multirow{2}{*}{$\frac{\text { DATE }}{05 / 20 / 06}$} & \multicolumn{3}{|c|}{$\begin{array}{l}\text { INSPECTION METHOD } \\
\text { IDENTIFICATION NUMBER }\end{array}$} & \multirow{2}{*}{$\begin{array}{l}\quad \text { REMARKS } \\
\text { Remote visual tank wall inspection } \\
\text { revealed no areas of concern since } \\
\text { last evaluated on } 9 / 3 / 05 \text {. }\end{array}$} \\
\hline & & P-01 & (A) & & WAP & l & P06125:01 & \\
\hline $\mathrm{F}$ & 34 & P-02 & (A) & $05 / 20 / 06$ & WAP & l & P06125:02 & $\begin{array}{l}\text { Remote visual tank wall inspection } \\
\text { revealed no areas of concern since } \\
\text { last evaluated on } 3 / 23 / 05 \text {. }\end{array}$ \\
\hline $\mathrm{F}$ & 34 & $\mathrm{P}-03$ & (A) & $05 / 21 / 06$ & DP & l & $P 06115: 01-25$ & $\begin{array}{l}\text { Remote visual tank wall inspection } \\
\text { revealed no areas of concern since } \\
\text { last evaluated on 9/3/05. }\end{array}$ \\
\hline$F$ & 34 & P-04 & (A) & 05/20/06 & WAP & I & P06125:04 & $\begin{array}{l}\text { Remote visual tank wall inspection } \\
\text { revealed no areas of concern since } \\
\text { last evaluated on } 9 / 3 / 05 \text {. }\end{array}$ \\
\hline$F$ & 34 & P-05 & (A) & 05/20/06 & WAP & I & P06125:05 & $\begin{array}{l}\text { Remote visual tank wall inspection } \\
\text { revealed no areas of concern since } \\
\text { last evaluated on } 3 / 23 / 05 \text {. }\end{array}$ \\
\hline$F$ & 34 & P-06 & (A) & 05/21/06 & DP & I & P06116:01-26 & $\begin{array}{l}\text { Remote visual tank wall inspection } \\
\text { revealed no areas of concern since } \\
\text { last evaluated on } 9 / 3 / 05 \text {. }\end{array}$ \\
\hline $\mathrm{F}$ & 34 & P-07 & (A) & 05/20/06 & WAP & l & P06125:07 & $\begin{array}{l}\text { Remote visual tank wall inspection } \\
\text { revealed no areas of concern since } \\
\text { last evaluated on } 9 / 3 / 05 \text {. }\end{array}$ \\
\hline $\mathrm{F}$ & 34 & P-08 & (A) & $05 / 20 / 06$ & WAP & l & P06125:08 & $\begin{array}{l}\text { Remote visual tank wall inspection } \\
\text { revealed no areas of concern since } \\
\text { last evaluated on } 3 / 23 / 05 \text {. }\end{array}$ \\
\hline$F$ & 34 & P-09 & (A) & $05 / 21 / 06$ & DP & I & P06117:01-26 & $\begin{array}{l}\text { Remote visual tank wall inspection } \\
\text { revealed no areas of concern since } \\
\text { last evaluated on } 9 / 3 / 05 \text {. }\end{array}$ \\
\hline $\mathrm{F}$ & 34 & P-10 & $(\mathrm{A})$ & 05/21/06 & DP & I & P06118:01-28 & $\begin{array}{l}\text { Remote visual tank wall inspection } \\
\text { revealed no areas of concern since } \\
\text { last evaluated on } 9 / 3 / 05 \text {. Stains and } \\
\text { marks observed on the primary } \\
\text { vessel wall were caused by the } \\
\text { inleakage of water. }\end{array}$ \\
\hline$F$ & 34 & $\mathrm{P}-11$ & (A) & 05/20/06 & WAP & l & P06125:14 & $\begin{array}{l}\text { Remote visual tank wall inspection } \\
\text { revealed no areas of concern since } \\
\text { last evaluated on } 9 / 3 / 05 \text {. }\end{array}$ \\
\hline $\mathrm{F}$ & 34 & $\mathrm{P}-12$ & (A) & 05/20/06 & WAP & l & P06125:15 & $\begin{array}{l}\text { Remote visual tank wall inspection } \\
\text { revealed no areas of concern since } \\
\text { last evaluated on } 3 / 23 / 05 \text {. }\end{array}$ \\
\hline $\mathrm{F}$ & 34 & $P-13$ & (A) & 05/21/06 & DP & l & P06119:01-26 & $\begin{array}{l}\text { Remote visual tank wall inspection } \\
\text { revealed no areas of concern since } \\
\text { last evaluated on } 9 / 3 / 05 \text {. }\end{array}$ \\
\hline
\end{tabular}




\begin{tabular}{|c|c|c|c|c|c|c|}
\hline AREA & $\begin{array}{c}\text { TANK OR } \\
\text { ANCILLARY }\end{array}$ & $\frac{\text { ACCESS OPENING }}{\text { (A OR I) }}$ & DATE & \multicolumn{3}{|c|}{$\begin{array}{l}\text { INSPECTION METHOD } \\
\text { IDENTIFICATION NUMBER }\end{array}$} \\
\hline $\mathrm{F}$ & 34 & $\mathrm{P}-14 \quad(\mathrm{~A})$ & $05 / 20 / 06$ & WAP & 1 & P06125:09 \\
\hline$F$ & 34 & P-15 (A) & $05 / 20 / 06$ & WAP & I & P06125:11 \\
\hline$F$ & 34 & $\mathrm{P}-16 \quad(\mathrm{~A})$ & 05/20/06 & WAP & I & P06125:12 \\
\hline $\mathrm{H}$ & 35 & $A-01 \quad(A)$ & $05 / 21 / 06$ & DP & I & P06105:01-26 \\
\hline $\mathrm{H}$ & 35 & $A-01 \quad(A)$ & $11 / 30 / 06$ & UT & 1 & UT-06-010 \\
\hline
\end{tabular}




\begin{tabular}{|c|c|c|c|c|c|c|c|}
\hline \multirow{2}{*}{$\begin{array}{l}\text { AREA } \\
\mathrm{H}\end{array}$} & \multirow{2}{*}{$\begin{array}{c}\begin{array}{c}\text { TANK OR } \\
\text { ANCILLARY }\end{array} \\
35\end{array}$} & $\frac{\text { ACCESS OPENING }}{\text { (A OR I) }}$ & \multirow{2}{*}{$\frac{\text { DATE }}{11 / 30 / 06}$} & \multicolumn{3}{|c|}{$\begin{array}{l}\text { INSPECTION METHOD } \\
\text { IDENTIFICATION NUMBER }\end{array}$} & \multirow[b]{2}{*}{$\begin{array}{l}\text { REMARKS } \\
\text { UT scanning was performed on a } \\
\text { vertical strip } 8.5 \text { inches wide for the } \\
\text { entire accessible height of the } \\
\text { secondary vessel wall. Reportable } \\
\text { thicknesses were detected in one of } \\
\text { four secondary wall plates as well as } \\
\text { the annulus floor. Analysis of the } \\
\text { data revealed no reportable cracking } \\
\text { or other thinning or pitting in the } \\
\text { primary wall. This information is } \\
\text { included in document WSRC-TR } \\
\text {-2007-00064. }\end{array}$} \\
\hline & & $\mathrm{P}-02 \quad(\mathrm{~A})$ & & UT & I & UT-06-009 & \\
\hline $\mathrm{H}$ & 35 & $\mathrm{P}-03 \quad(\mathrm{~A})$ & $03 / 12 / 06$ & WAP & I & P06043:03 & $\begin{array}{l}\text { Remote visual tank wall inspection } \\
\text { revealed no areas of concern since } \\
\text { last evaluated on } 1 / 8 / 05 \text {. }\end{array}$ \\
\hline $\mathrm{H}$ & 35 & $\mathrm{P}-04 \quad(\mathrm{~A})$ & $03 / 12 / 06$ & WAP & I & P06043:04 & $\begin{array}{l}\text { Remote visual tank wall inspection } \\
\text { revealed no areas of concern since } \\
\text { last evaluated on } 1 / 8 / 05\end{array}$ \\
\hline $\mathrm{H}$ & 35 & $\mathrm{P}-05 \quad(\mathrm{~A})$ & $03 / 12 / 06$ & WAP & I & P06043:05 & $\begin{array}{l}\text { Remote visual tank wall inspection } \\
\text { revealed no areas of concern since } \\
\text { last evaluated on } 1 / 11 / 05 \text {. The } \\
\text { annulus jet beneath the } F \text { Riser } \\
\text { showed no degradation per T-DS-G } \\
-00043 \text {. }\end{array}$ \\
\hline
\end{tabular}

$\mathrm{H}$

35

35

35

35

P-09 (A)

$03 / 12 / 06$

35

35

35

$\mathrm{P}-06 \quad(\mathrm{~A})$

$\mathrm{P}-07 \quad(\mathrm{~A})$

$\mathrm{P}-08 \quad(\mathrm{~A})$

$03 / 12 / 06$

WAP

I P06043:08

$\mathrm{H}$

$\mathrm{H}$

$\mathrm{H}$

$03 / 12 / 06$

WAP

I

P06043:10

P-11 (A)

$03 / 12 / 06$

WAP

I P06043:11

$\mathrm{P}-12 \quad(\mathrm{~A})$

$03 / 12 / 06$

WAP

P06043:12
Remote visual tank wall inspection revealed no areas of concern since last evaluated on 1/8/05.

Remote visual tank wall inspection revealed no areas of concern since last evaluated on 1/11/05.

Remote visual tank wall inspection revealed no areas of concern since last evaluated on 1/8/05.

Remote visual tank wall inspection revealed no areas of concern since last evaluated on 1/11/05.

Remote visual tank wall inspection revealed no areas of concern since last evaluated on 1/8/05.

Remote visual tank wall inspection last evaluated on 1/11/05.

Remote visual tank wall inspection revealed no areas of concern since last evaluated on 1/8/05. revealed no areas of concern since 


\begin{tabular}{|c|c|c|c|c|c|c|c|c|}
\hline \multirow{2}{*}{ AREA } & \multirow{2}{*}{ 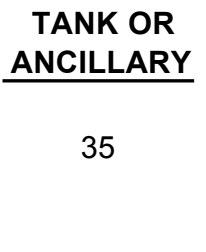 } & \multicolumn{2}{|c|}{$\begin{array}{c}\text { ACCESS OPENING } \\
\text { (A OR I) }\end{array}$} & \multirow{2}{*}{$\begin{array}{l}\text { DATE } \\
03 / 12 / 06\end{array}$} & \multicolumn{3}{|c|}{$\begin{array}{l}\text { INSPECTION METHOD } \\
\text { IDENTIFICATION NUMBER }\end{array}$} & \multirow{2}{*}{$\begin{array}{l}\qquad \text { REMARKS } \\
\text { Remote visual tank wall inspection } \\
\text { revealed no areas of concern since } \\
\text { last evaluated on } 1 / 11 / 05 \text {. }\end{array}$} \\
\hline & & P-13 & (A) & & WAP & l & P06043:13 & \\
\hline $\mathrm{H}$ & 35 & P-14 & (A) & $03 / 12 / 06$ & WAP & l & P06043:14 & $\begin{array}{l}\text { Remote visual tank wall inspection } \\
\text { revealed no areas of concern since } \\
\text { last evaluated on } 1 / 8 / 05 \text {. }\end{array}$ \\
\hline $\mathrm{H}$ & 36 & A-01 & (A) & $05 / 21 / 06$ & $\mathrm{DP}$ & I & P06170:01-27 & $\begin{array}{l}\text { Remote visual tank wall inspection } \\
\text { revealed no areas of concern since } \\
\text { last evaluated on } 1 / 8 / 05 \text {. }\end{array}$ \\
\hline $\mathrm{H}$ & 36 & A-02 & (A) & $05 / 21 / 06$ & $\mathrm{DP}$ & l & P06171:01-27 & $\begin{array}{l}\text { Remote visual tank wall inspection } \\
\text { revealed no areas of concern since } \\
\text { last evaluated on } 1 / 8 / 05 \text {. }\end{array}$ \\
\hline $\mathrm{H}$ & 36 & A-02 & (A) & $05 / 21 / 06$ & $\mathrm{DP}$ & l & P06171:21 & $\begin{array}{l}\text { The conductivity probe was properly } \\
\text { positioned per J-JX-G-0001. }\end{array}$ \\
\hline $\mathrm{H}$ & 36 & A-03 & (A) & $05 / 21 / 06$ & $\mathrm{DP}$ & I & P06172:01-27 & $\begin{array}{l}\text { Remote visual tank wall inspection } \\
\text { revealed no areas of concern since } \\
\text { last evaluated on } 1 / 8 / 05 \text {. }\end{array}$ \\
\hline $\mathrm{H}$ & 36 & A-03 & (A) & $05 / 21 / 06$ & $\mathrm{DP}$ & l & P06172:21 & $\begin{array}{l}\text { The conductivity probe was properly } \\
\text { positioned per J-JX-G-0001. }\end{array}$ \\
\hline $\mathrm{H}$ & 36 & A-04 & (A) & 07/04/06 & $\mathrm{DP}$ & l & P06174:01-28 & $\begin{array}{l}\text { Remote visual tank wall inspection } \\
\text { revealed no areas of concern since } \\
\text { last evaluated on } 1 / 08 / 05 \text {. }\end{array}$ \\
\hline $\mathrm{H}$ & 36 & A-04 & (A) & 07/04/06 & $\mathrm{DP}$ & I & P06174:23 & $\begin{array}{l}\text { The conductivity probe was properly } \\
\text { positioned per J-JX-G-0001. }\end{array}$ \\
\hline $\mathrm{H}$ & 36 & P-01 & (A) & $01 / 25 / 06$ & WAP & I & P06005:01 & $\begin{array}{l}\text { Remote visual tank wall inspection } \\
\text { revealed no areas of concern since } \\
\text { last evaluated on } 1 / 8 / 05 \text {. }\end{array}$ \\
\hline $\mathrm{H}$ & 36 & P-02 & (A) & $01 / 25 / 06$ & WAP & I & P06005:02 & $\begin{array}{l}\text { Remote visual tank wall inspection } \\
\text { revealed no areas of concern since } \\
\text { last evaluated on } 1 / 8 / 05 \text {. }\end{array}$ \\
\hline $\mathrm{H}$ & 36 & P-03 & (A) & $01 / 25 / 06$ & WAP & I & P06005:03 & $\begin{array}{l}\text { Remote visual tank wall inspection } \\
\text { revealed no areas of concern since } \\
\text { last evaluated on } 1 / 8 / 05 \text {. }\end{array}$ \\
\hline $\mathrm{H}$ & 36 & P-04 & (A) & $01 / 25 / 06$ & WAP & I & P06005:04 & $\begin{array}{l}\text { Remote visual tank wall inspection } \\
\text { revealed no areas of concern since } \\
\text { last evaluated on } 1 / 8 / 05 \text {. }\end{array}$ \\
\hline $\mathrm{H}$ & 36 & P-05 & (A) & $01 / 25 / 06$ & WAP & I & P06005:05 & $\begin{array}{l}\text { Remote visual tank wall inspection } \\
\text { revealed no areas of concern since } \\
\text { last evaluated on } 1 / 9 / 05 \text {. }\end{array}$ \\
\hline $\mathrm{H}$ & 36 & P-06 & (A) & $01 / 25 / 06$ & WAP & I & P06005:06 & $\begin{array}{l}\text { Remote visual tank wall inspection } \\
\text { revealed no areas of concern since } \\
\text { last evaluated on } 1 / 8 / 05 \text {. }\end{array}$ \\
\hline
\end{tabular}




\begin{tabular}{|c|c|c|c|c|c|c|c|c|}
\hline \multirow{2}{*}{ AREA } & \multirow{2}{*}{$\begin{array}{c}\begin{array}{c}\text { TANK OR } \\
\text { ANCILLARY }\end{array} \\
36\end{array}$} & \multicolumn{2}{|c|}{$\begin{array}{c}\text { ACCESS OPENING } \\
\text { (A OR I) }\end{array}$} & \multirow{2}{*}{$\frac{\text { DATE }}{01 / 25 / 06}$} & \multicolumn{3}{|c|}{$\begin{array}{l}\text { INSPECTION METHOD } \\
\text { IDENTIFICATION NUMBER }\end{array}$} & \multirow{2}{*}{$\begin{array}{l}\text { REMARKS } \\
\text { Remote visual tank wall inspection } \\
\text { revealed no areas of concern since } \\
\text { last evaluated on } 1 / 9 / 05 \text {. }\end{array}$} \\
\hline & & P-07 & (A) & & WAP & l & P06005:07 & \\
\hline $\mathrm{H}$ & 36 & P-08 & (A) & $01 / 25 / 06$ & WAP & l & P06005:08 & $\begin{array}{l}\text { Remote visual tank wall inspection } \\
\text { revealed no areas of concern since } \\
\text { last evaluated on } 1 / 8 / 05\end{array}$ \\
\hline $\mathrm{H}$ & 36 & P-09 & (A) & $01 / 25 / 06$ & WAP & l & P06005:09 & $\begin{array}{l}\text { Remote visual tank wall inspection } \\
\text { revealed no areas of concern since } \\
\text { last evaluated on } 1 / 9 / 05 \text {. }\end{array}$ \\
\hline $\mathrm{H}$ & 36 & P-10 & (A) & $01 / 25 / 06$ & WAP & l & P06005:10 & $\begin{array}{l}\text { Remote visual tank wall inspection } \\
\text { revealed no areas of concern since } \\
\text { last evaluated on } 1 / 8 / 05\end{array}$ \\
\hline $\mathrm{H}$ & 36 & $\mathrm{P}-11$ & (A) & $01 / 25 / 06$ & WAP & l & P06005:11 & $\begin{array}{l}\text { Remote visual tank wall inspection } \\
\text { revealed no areas of concern since } \\
\text { last evaluated on } 1 / 9 / 05 \text {. }\end{array}$ \\
\hline $\mathrm{H}$ & 36 & P-12 & (A) & $01 / 25 / 06$ & WAP & I & P06005:12 & $\begin{array}{l}\text { Remote visual tank wall inspection } \\
\text { revealed no areas of concern since } \\
\text { last evaluated on } 1 / 8 / 05 \text {. }\end{array}$ \\
\hline $\mathrm{H}$ & 36 & $P-13$ & (A) & $01 / 25 / 06$ & WAP & I & P06005:13 & $\begin{array}{l}\text { Remote visual tank wall inspection } \\
\text { revealed no areas of concern since } \\
\text { last evaluated on } 1 / 9 / 05 \text {. The } \\
\text { annulus jet beneath the } F \text { riser } \\
\text { showed no degradation per T-DS-G } \\
-00043 \text {. }\end{array}$ \\
\hline $\mathrm{H}$ & 36 & P-14 & (A) & $01 / 25 / 06$ & WAP & l & P06005:14 & $\begin{array}{l}\text { Remote visual tank wall inspection } \\
\text { revealed no areas of concern since } \\
\text { last evaluated on } 1 / 8 / 05 \text {. }\end{array}$ \\
\hline $\mathrm{H}$ & 37 & COP \#106 & & 06/26/06 & DSP & I & P06160:01-06 & $\begin{array}{l}\text { Inspection of the transfer lines within } \\
\text { the clean out port revealed no } \\
\text { degradation per T-DS-G-00001. }\end{array}$ \\
\hline $\mathrm{H}$ & 37 & COP \#107 & & 06/26/06 & DSP & I & P06161:01-06 & $\begin{array}{l}\text { Inspection of the transfer lines within } \\
\text { the clean out port revealed no } \\
\text { degradation per T-DS-G-00001. }\end{array}$ \\
\hline $\mathrm{H}$ & 37 & COP \#108 & & 06/26/06 & DSP & I & P06162:01-06 & $\begin{array}{l}\text { Inspection of the transfer lines within } \\
\text { the clean out port revealed no } \\
\text { degradation per T-DS-G-00001. }\end{array}$ \\
\hline $\mathrm{H}$ & 37 & A-01 & $(\mathrm{A})$ & $07 / 04 / 06$ & $\mathrm{DP}$ & l & P06187:01-27 & $\begin{array}{l}\text { Remote visual tank wall inspection } \\
\text { revealed no areas of concern since } \\
\text { last evaluated on } 1 / 08 / 05 \text {. }\end{array}$ \\
\hline $\mathrm{H}$ & 37 & A-02 & (A) & $07 / 04 / 06$ & $\mathrm{DP}$ & l & P06188:01-27 & $\begin{array}{l}\text { Remote visual tank wall inspection } \\
\text { revealed no areas of concern since } \\
\text { last evaluated on } 1 / 8 / 05 \text {. }\end{array}$ \\
\hline
\end{tabular}




\begin{tabular}{|c|c|c|c|c|c|c|c|c|}
\hline \multirow{2}{*}{ AREA } & \multirow{2}{*}{$\begin{array}{c}\begin{array}{c}\text { TANK OR } \\
\text { ANCILLARY }\end{array} \\
\frac{37}{37}\end{array}$} & \multicolumn{2}{|c|}{$\begin{array}{c}\text { ACCESS OPENING } \\
\text { (A OR I) }\end{array}$} & \multirow{2}{*}{$\frac{\text { DATE }}{07 / 04 / 06}$} & \multicolumn{3}{|c|}{$\begin{array}{l}\text { INSPECTION METHOD } \\
\text { IDENTIFICATION NUMBER }\end{array}$} & \multirow{2}{*}{$\begin{array}{l}\text { REMARKS } \\
\text { The conductivity probe was properly } \\
\text { positioned per J-JX-G-0001. }\end{array}$} \\
\hline & & A-02 & (A) & & $\mathrm{DP}$ & l & P06188:12 & \\
\hline $\mathrm{H}$ & 37 & A-03 & (A) & 07/04/06 & $\mathrm{DP}$ & l & P06189:01-27 & $\begin{array}{l}\text { Remote visual tank wall inspection } \\
\text { revealed no areas of concern since } \\
\text { last evaluated on } 1 / 08 / 05 \text {. }\end{array}$ \\
\hline $\mathrm{H}$ & 37 & A-03 & (A) & $07 / 04 / 06$ & $\mathrm{DP}$ & l & P06189:22 & $\begin{array}{l}\text { The conductivity probe was properly } \\
\text { positioned per J-JX-G-0001. }\end{array}$ \\
\hline $\mathrm{H}$ & 37 & A-04 & (A) & 07/04/06 & $\mathrm{DP}$ & I & P06190:22 & $\begin{array}{l}\text { The conductivity probe was properly } \\
\text { positioned per J-JX-G-0001. }\end{array}$ \\
\hline $\mathrm{H}$ & 37 & A-04 & (A) & 07/04/06 & $\mathrm{DP}$ & l & P06190:01-27 & $\begin{array}{l}\text { Remote visual tank wall inspection } \\
\text { revealed no areas of concern since } \\
\text { last evaluated on } 1 / 08 / 05 \text {. }\end{array}$ \\
\hline $\mathrm{H}$ & 37 & P-01 & (A) & 03/12/06 & WAP & l & P06042:01 & $\begin{array}{l}\text { Remote visual tank wall inspection } \\
\text { revealed no areas of concern since } \\
\text { last evaluated on } 1 / 8 / 05 \text {. }\end{array}$ \\
\hline $\mathrm{H}$ & 37 & P-02 & (A) & $03 / 12 / 06$ & WAP & l & P06042:02 & $\begin{array}{l}\text { Remote visual tank wall inspection } \\
\text { revealed no areas of concern since } \\
\text { last evaluated on } 1 / 8 / 05 \text {. }\end{array}$ \\
\hline $\mathrm{H}$ & 37 & P-03 & (A) & $03 / 12 / 06$ & WAP & I & P06042:03 & $\begin{array}{l}\text { Remote visual tank wall inspection } \\
\text { revealed no areas of concern since } \\
\text { last evaluated on } 1 / 8 / 05 \text {. }\end{array}$ \\
\hline $\mathrm{H}$ & 37 & P-04 & (A) & 03/12/06 & WAP & l & P06042:04 & $\begin{array}{l}\text { Remote visual tank wall inspection } \\
\text { revealed no areas of concern since } \\
\text { last evaluated on } 1 / 8 / 05 \text {. }\end{array}$ \\
\hline $\mathrm{H}$ & 37 & P-05 & (A) & 03/12/06 & WAP & I & P06042:05 & $\begin{array}{l}\text { Remote visual tank wall inspection } \\
\text { revealed no areas of concern since } \\
\text { last evaluated on } 1 / 12 / 05 \text {. }\end{array}$ \\
\hline $\mathrm{H}$ & 37 & P-06 & (A) & 03/12/06 & WAP & I & P06042:06 & $\begin{array}{l}\text { Remote visual tank wall inspection } \\
\text { revealed no areas of concern since } \\
\text { last evaluated on } 1 / 8 / 05 \text {. }\end{array}$ \\
\hline $\mathrm{H}$ & 37 & P-07 & (A) & $03 / 12 / 06$ & WAP & l & P06042:07 & $\begin{array}{l}\text { Remote visual tank wall inspection } \\
\text { revealed no areas of concern since } \\
\text { last evaluated on } 1 / 12 / 05 \text {. }\end{array}$ \\
\hline $\mathrm{H}$ & 37 & P-08 & (A) & 03/12/06 & WAP & l & P06042:08 & $\begin{array}{l}\text { Remote visual tank wall inspection } \\
\text { revealed no areas of concern since } \\
\text { last evaluated on } 1 / 8 / 05 \text {. }\end{array}$ \\
\hline $\mathrm{H}$ & 37 & P-09 & (A) & $03 / 12 / 06$ & WAP & l & P06042:09 & $\begin{array}{l}\text { Remote visual tank wall inspection } \\
\text { revealed no areas of concern since } \\
\text { last evaluated on } 1 / 12 / 05 \text {. }\end{array}$ \\
\hline $\mathrm{H}$ & 37 & P-10 & (A) & $03 / 12 / 06$ & WAP & I & P06042:10 & $\begin{array}{l}\text { Remote visual tank wall inspection } \\
\text { revealed no areas of concern since } \\
\text { last evaluated on } 1 / 8 / 05 \text {. }\end{array}$ \\
\hline
\end{tabular}




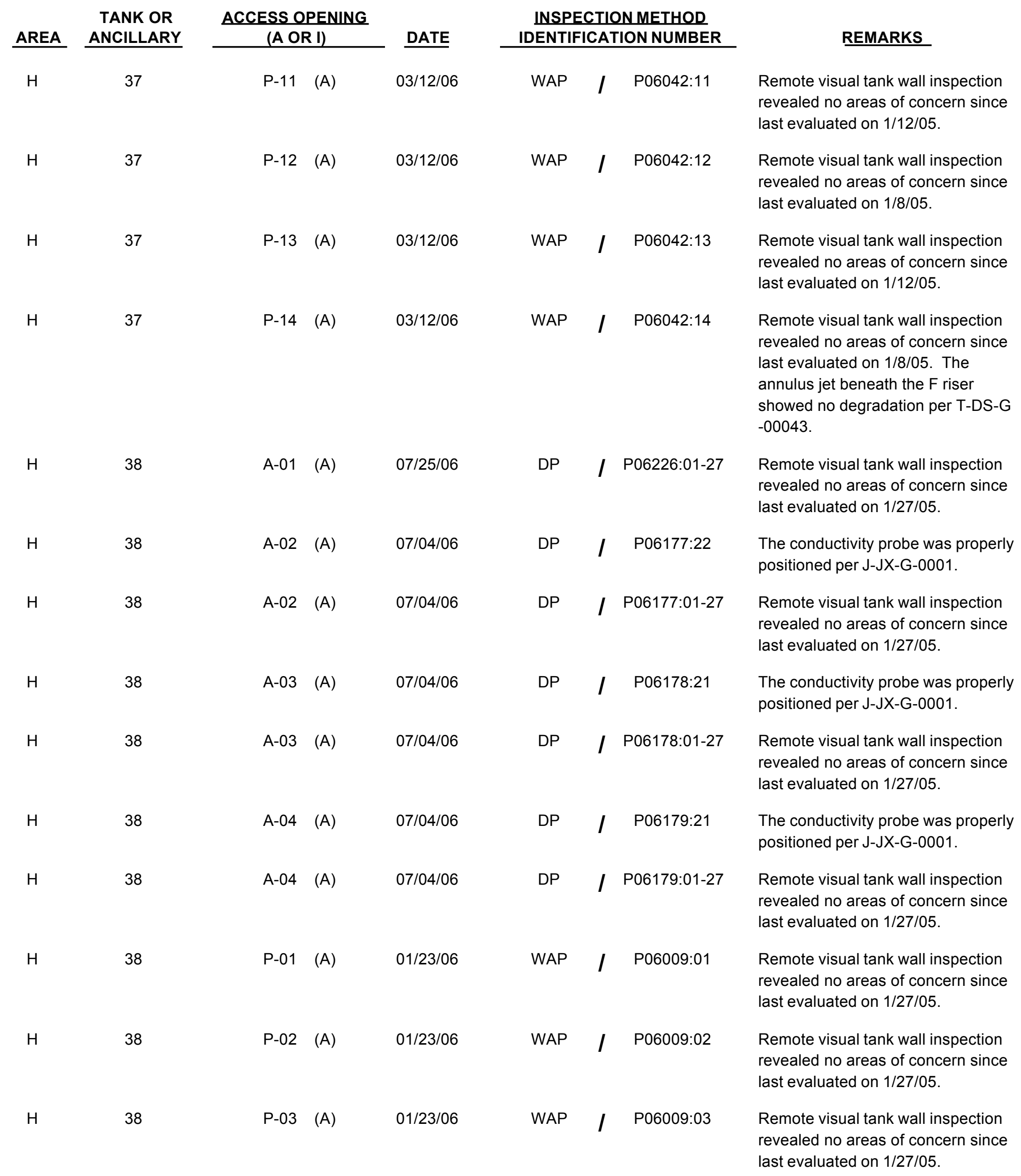




\begin{tabular}{|c|c|c|c|c|c|c|c|c|}
\hline \multirow{2}{*}{$\begin{array}{l}\text { AREA } \\
\mathrm{H}\end{array}$} & \multirow{2}{*}{$\begin{array}{c}\begin{array}{c}\text { TANK OR } \\
\text { ANCILLARY }\end{array} \\
38\end{array}$} & \multicolumn{2}{|c|}{$\frac{\text { ACCESS OPENING }}{(\text { A OR I) }}$} & \multirow{2}{*}{$\begin{array}{l}\text { DATE } \\
01 / 23 / 06\end{array}$} & \multicolumn{3}{|c|}{$\begin{array}{l}\text { INSPECTION METHOD } \\
\text { IDENTIFICATION NUMBER }\end{array}$} & \multirow{2}{*}{$\begin{array}{l}\text { REMARKS } \\
\text { Remote visual tank wall inspection } \\
\text { revealed no areas of concern since } \\
\text { last evaluated on } 1 / 27 / 05 \text {. }\end{array}$} \\
\hline & & $\mathrm{P}-04$ & (A) & & WAP & l & P06009:04 & \\
\hline $\mathrm{H}$ & 38 & P-05 & (A) & $01 / 23 / 06$ & WAP & l & P06009:05 & $\begin{array}{l}\text { Remote visual tank wall inspection } \\
\text { revealed no areas of concern since } \\
\text { last evaluated on } 1 / 27 / 05 \text {. }\end{array}$ \\
\hline $\mathrm{H}$ & 38 & P-06 & (A) & $01 / 23 / 06$ & WAP & l & P06009:06 & $\begin{array}{l}\text { Remote visual tank wall inspection } \\
\text { revealed no areas of concern since } \\
\text { last evaluated on } 1 / 27 / 05 \text {. }\end{array}$ \\
\hline $\mathrm{H}$ & 38 & P-07 & (A) & $01 / 23 / 06$ & WAP & 1 & P06009:07 & $\begin{array}{l}\text { Remote visual tank wall inspection } \\
\text { revealed no areas of concern since } \\
\text { last evaluated on } 1 / 27 / 05 \text {. }\end{array}$ \\
\hline $\mathrm{H}$ & 38 & P-08 & (A) & $01 / 23 / 06$ & WAP & l & P06009:08 & $\begin{array}{l}\text { Remote visual tank wall inspection } \\
\text { revealed no areas of concern since } \\
\text { last evaluated on } 1 / 27 / 05 \text {. }\end{array}$ \\
\hline $\mathrm{H}$ & 38 & P-09 & (A) & $01 / 23 / 06$ & WAP & l & P06009:09 & $\begin{array}{l}\text { Remote visual tank wall inspection } \\
\text { revealed no areas of concern since } \\
\text { last evaluated on } 1 / 27 / 05 \text {. }\end{array}$ \\
\hline $\mathrm{H}$ & 38 & $P-10$ & (A) & $01 / 23 / 06$ & WAP & l & P06009:10 & $\begin{array}{l}\text { Remote visual tank wall inspection } \\
\text { revealed no areas of concern since } \\
\text { last evaluated on } 2 / 7 / 05 \text {. }\end{array}$ \\
\hline $\mathrm{H}$ & 38 & $P-11$ & (A) & $01 / 23 / 06$ & WAP & l & P06009:11 & $\begin{array}{l}\text { Remote visual tank wall inspection } \\
\text { revealed no areas of concern since } \\
\text { last evaluated on } 2 / 7 / 05 \text {. Stains on } \\
\text { the secondary vessel wall were } \\
\text { caused by the inleakage of water. }\end{array}$ \\
\hline $\mathrm{H}$ & 38 & P-12 & (A) & $01 / 23 / 06$ & WAP & l & P06009:12 & $\begin{array}{l}\text { Remote visual tank wall inspection } \\
\text { revealed no areas of concern since } \\
\text { last evaluated on } 2 / 7 / 05 \text {. The } \\
\text { annulus jet beneath the F riser } \\
\text { showed no degradation per T-DS-G } \\
-00043 \text {. }\end{array}$ \\
\hline $\mathrm{H}$ & 38 & $P-13$ & (A) & $01 / 23 / 06$ & WAP & l & P06009:13 & $\begin{array}{l}\text { Remote visual tank wall inspection } \\
\text { revealed no areas of concern since } \\
\text { last evaluated on } 2 / 7 / 05 \text {. }\end{array}$ \\
\hline $\mathrm{H}$ & 38 & P-14 & $(\mathrm{A})$ & $01 / 23 / 06$ & WAP & l & P06009:14 & $\begin{array}{l}\text { Remote visual tank wall inspection } \\
\text { revealed no areas of concern since } \\
\text { last evaluated on } 2 / 7 / 05 \text {. }\end{array}$ \\
\hline $\mathrm{H}$ & 39 & $A-01$ & $(\mathrm{~A})$ & $07 / 04 / 06$ & DP & l & P06183:01-27 & $\begin{array}{l}\text { Remote visual tank wall inspection } \\
\text { revealed no areas of concern since } \\
\text { last evaluated on } 1 / 27 / 05 \text {. }\end{array}$ \\
\hline
\end{tabular}




\begin{tabular}{|c|c|c|c|c|c|c|c|c|}
\hline \multirow{2}{*}{ AREA } & \multirow{2}{*}{$\begin{array}{c}\begin{array}{c}\text { TANK OR } \\
\text { ANCILLARY }\end{array} \\
39\end{array}$} & \multicolumn{2}{|c|}{$\begin{array}{c}\text { ACCESS OPENING } \\
\text { (A OR I) }\end{array}$} & \multirow{2}{*}{$\frac{\text { DATE }}{07 / 04 / 06}$} & \multicolumn{3}{|c|}{$\begin{array}{l}\text { INSPECTION METHOD } \\
\text { IDENTIFICATION NUMBER }\end{array}$} & \multirow{2}{*}{$\begin{array}{l}\text { REMARKS } \\
\text { Remote visual tank wall inspection } \\
\text { revealed no areas of concern since } \\
\text { last evaluated on } 1 / 27 / 05 \text {. }\end{array}$} \\
\hline & & A-02 & (A) & & $\mathrm{DP}$ & I & P06184:01-27 & \\
\hline $\mathrm{H}$ & 39 & A-02 & (A) & 07/04/06 & DP & I & P06184:22 & $\begin{array}{l}\text { The conductivity probe was properly } \\
\text { positioned per J-JX-G-0001. }\end{array}$ \\
\hline $\mathrm{H}$ & 39 & A-03 & (A) & $07 / 04 / 06$ & $\mathrm{DP}$ & I & P06185:01-27 & $\begin{array}{l}\text { Remote visual tank wall inspection } \\
\text { revealed no areas of concern since } \\
\text { last evaluated on } 1 / 27 / 05 \text {. }\end{array}$ \\
\hline $\mathrm{H}$ & 39 & A-03 & (A) & 07/04/06 & DP & I & P06185:22 & $\begin{array}{l}\text { The conductivity probe was properly } \\
\text { positioned per J-JX-G-0001. }\end{array}$ \\
\hline $\mathrm{H}$ & 39 & A-04 & (A) & 07/04/06 & $\mathrm{DP}$ & I & P06186:01-27 & $\begin{array}{l}\text { Remote visual tank wall inspection } \\
\text { revealed no areas of concern since } \\
\text { last evaluated on } 1 / 27 / 05 \text {. }\end{array}$ \\
\hline $\mathrm{H}$ & 39 & A- 04 & (A) & 07/04/06 & $\mathrm{DP}$ & I & P06186:22 & $\begin{array}{l}\text { The conductivity probe was properly } \\
\text { positioned per J-JX-G-0001. }\end{array}$ \\
\hline $\mathrm{H}$ & 39 & P-01 & (A) & $01 / 23 / 06$ & WAP & I & P06007:01 & $\begin{array}{l}\text { Remote visual tank wall inspection } \\
\text { revealed no areas of concern since } \\
\text { last evaluated on } 1 / 27 / 05 \text {. The } \\
\text { annulus jet beneath the } F \text { riser } \\
\text { showed no degradation per T-DS-G } \\
-00043 \text {. }\end{array}$ \\
\hline $\mathrm{H}$ & 39 & P-02 & (A) & $01 / 23 / 06$ & WAP & I & P06007:02 & $\begin{array}{l}\text { Remote visual tank wall inspection } \\
\text { revealed no areas of concern since } \\
\text { last evaluated on } 1 / 27 / 05 \text {. }\end{array}$ \\
\hline $\mathrm{H}$ & 39 & P-03 & (A) & $01 / 23 / 06$ & WAP & I & P06007:03 & $\begin{array}{l}\text { Remote visual tank wall inspection } \\
\text { revealed no areas of concern since } \\
\text { last evaluated on } 1 / 27 / 05 \text {. }\end{array}$ \\
\hline $\mathrm{H}$ & 39 & P-04 & (A) & 01/23/06 & WAP & I & P06007:04 & $\begin{array}{l}\text { Remote visual tank wall inspection } \\
\text { revealed no areas of concern since } \\
\text { last evaluated on } 1 / 27 / 05 \text {. }\end{array}$ \\
\hline $\mathrm{H}$ & 39 & P-05 & (A) & $01 / 23 / 06$ & WAP & I & P06007:05 & $\begin{array}{l}\text { Remote visual tank wall inspection } \\
\text { revealed no areas of concern since } \\
\text { last evaluated on } 1 / 27 / 05 \text {. Stains on } \\
\text { the secondary vessel wall were } \\
\text { caused by the inleakage of water. }\end{array}$ \\
\hline $\mathrm{H}$ & 39 & P-06 & (A) & $01 / 23 / 06$ & WAP & I & P06007:06 & $\begin{array}{l}\text { Remote visual tank wall inspection } \\
\text { revealed no areas of concern since } \\
\text { last evaluated on } 1 / 27 / 05 \text {. }\end{array}$ \\
\hline $\mathrm{H}$ & 39 & P-07 & (A) & 01/23/06 & WAP & I & P06007:07 & $\begin{array}{l}\text { Remote visual tank wall inspection } \\
\text { revealed no areas of concern since } \\
\text { last evaluated on } 1 / 27 / 05 \text {. }\end{array}$ \\
\hline
\end{tabular}




\begin{tabular}{|c|c|c|c|c|c|c|c|c|}
\hline $\begin{array}{l}\text { AREA } \\
\mathrm{H}\end{array}$ & \multirow{2}{*}{$\begin{array}{c}\text { TANK OR } \\
\text { ANCILLARY } \\
39\end{array}$} & \multicolumn{2}{|c|}{$\frac{\text { ACCESS OPENING }}{\text { (A OR I) }}$} & $\begin{array}{l}\text { DATE } \\
01 / 23 / 06\end{array}$ & \multicolumn{3}{|c|}{$\begin{array}{l}\text { INSPECTION METHOD } \\
\text { IDENTIFICATION NUMBER }\end{array}$} & \multirow{2}{*}{$\begin{array}{l}\text { REMARKS } \\
\text { Remote visual tank wall inspection } \\
\text { revealed no areas of concern since } \\
\text { last evaluated on } 1 / 27 / 05 \text {. Stains on } \\
\text { the secondary vessel wall were } \\
\text { caused by the inleakage of water. }\end{array}$} \\
\hline $\mathrm{H}$ & & P-08 & (A) & $01 / 23 / 06$ & WAP & I & P06007:08 & \\
\hline $\mathrm{H}$ & 39 & P-09 & (A) & 01/23/06 & WAP & I & P06007:09 & $\begin{array}{l}\text { Remote visual tank wall inspection } \\
\text { revealed no areas of concern since } \\
\text { last evaluated on } 1 / 27 / 05 \text {. Stains on } \\
\text { the secondary vessel wall were } \\
\text { caused by the inleakage of water. }\end{array}$ \\
\hline $\mathrm{H}$ & 39 & $P-10$ & (A) & 01/23/06 & WAP & I & P06007:10 & $\begin{array}{l}\text { Remote visual tank wall inspection } \\
\text { revealed no areas of concern since } \\
\text { last evaluated on } 1 / 27 / 05 \text {. }\end{array}$ \\
\hline $\mathrm{H}$ & 39 & P-11 & $(\mathrm{A})$ & $01 / 23 / 06$ & WAP & I & P06007:11 & $\begin{array}{l}\text { Remote visual tank wall inspection } \\
\text { revealed no areas of concern since } \\
\text { last evaluated on } 2 / 9 / 05 \text {. }\end{array}$ \\
\hline $\mathrm{H}$ & 39 & $\mathrm{P}-12$ & (A) & $01 / 23 / 06$ & WAP & I & P06007:12 & $\begin{array}{l}\text { Remote visual tank wall inspection } \\
\text { revealed no areas of concern since } \\
\text { last evaluated on } 2 / 9 / 05 \text {. }\end{array}$ \\
\hline $\mathrm{H}$ & 39 & $P-13$ & $(\mathrm{~A})$ & $01 / 23 / 06$ & WAP & I & P06007:13 & $\begin{array}{l}\text { Remote visual tank wall inspection } \\
\text { revealed no areas of concern since } \\
\text { last evaluated on } 2 / 9 / 05 \text {. }\end{array}$ \\
\hline $\mathrm{H}$ & 39 & P-14 & (A) & 01/23/06 & WAP & I & P06007:14 & $\begin{array}{l}\text { Remote visual tank wall inspection } \\
\text { revealed no areas of concern since } \\
\text { last evaluated on } 2 / 9 / 05 \text {. Stains on } \\
\text { the secondary vessel wall were } \\
\text { caused by the inleakage of water. }\end{array}$ \\
\hline $\mathrm{H}$ & 40 & A-01 & (A) & $01 / 24 / 06$ & WAP & I & P06006:12 & $\begin{array}{l}\text { Remote visual tank wall inspection } \\
\text { revealed no areas of concern since } \\
\text { last evaluated on } 2 / 11 / 05 \text {. }\end{array}$ \\
\hline $\mathrm{H}$ & 40 & A-02 & (A) & $01 / 24 / 06$ & WAP & I & P06006:01 & $\begin{array}{l}\text { Remote visual tank wall inspection } \\
\text { revealed no areas of concern since } \\
\text { last evaluated on } 2 / 11 / 05 \text {. }\end{array}$ \\
\hline $\mathrm{H}$ & 40 & A-03 & (A) & $01 / 24 / 06$ & WAP & I & P06006:05 & $\begin{array}{l}\text { Remote visual tank wall inspection } \\
\text { revealed no areas of concern since } \\
\text { last evaluated on } 2 / 11 / 05 \text {. }\end{array}$ \\
\hline $\mathrm{H}$ & 40 & A-04 & (A) & $01 / 24 / 06$ & WAP & I & P06006:08 & $\begin{array}{l}\text { Remote visual tank wall inspection } \\
\text { revealed no areas of concern since } \\
\text { last evaluated on } 2 / 10 / 05 \text {. }\end{array}$ \\
\hline
\end{tabular}




\begin{tabular}{|c|c|c|c|c|c|c|c|}
\hline \multirow{2}{*}{$\frac{\text { AREA }}{\mathrm{H}}$} & \multirow{2}{*}{ 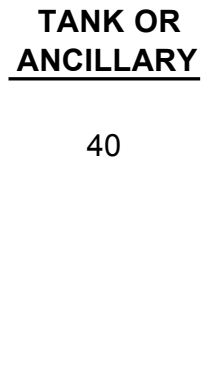 } & $\frac{\text { ACCESS OPENING }}{\text { (A OR I) }}$ & \multirow{2}{*}{$\begin{array}{c}\underline{\text { DATE }} \\
01 / 24 / 06\end{array}$} & \multicolumn{3}{|c|}{$\begin{array}{l}\text { INSPECTION METHOD } \\
\text { IDENTIFICATION NUMBER }\end{array}$} & \multirow{2}{*}{$\begin{array}{l}\quad \text { REMARKS } \\
\text { Remote visual tank wall inspection } \\
\text { revealed no areas of concern since } \\
\text { last evaluated on } 3 / 5 / 05 \text {. The } \\
\text { annulus jet beneath the } \mathrm{F} \text { riser } \\
\text { showed no degradation per T-DS-G } \\
\text {-00043. }\end{array}$} \\
\hline & & $\mathrm{P}-01 \quad$ (A) & & WAP & I & P06006:11 & \\
\hline
\end{tabular}

$\mathrm{H}$

40

P-01 (A)

09/20/06

DP

I P06269:01-27

$\mathrm{H}$

40

P-02 (A)

$12 / 06 / 06$

$\mathrm{H}$

$\mathrm{H}$

$\mathrm{H}$

40

40

$\mathrm{H}$

40

$\mathrm{H}$

40

$\mathrm{H}$

40
P-05 (A)

07/15/06

P-06 (A)

01/24/06

$\mathrm{P}-03 \quad(\mathrm{~A})$

$\mathrm{P}-04 \quad(\mathrm{~A})$

P-05 (A)

07/15/06

P-07 (A)

P-08 (A)

07/15/06
$01 / 24 / 06$

WAP

I P06006:04

DP
WAP I P06006:13

WAP

I P06006:02

DP

I P06217:01-27

DP I P06218:01-28

WAP I P06006:03

DP
Remote visual tank wall inspection revealed no areas of concern since

Remote visual tank wall inspection revealed no areas of concern since last evaluated on $2 / 10 / 05$. The annulus jet beneath the $F$ riser showed no degradation per T-DS-G -00043. Dried gilsulate was observed on the annulus floor. This material appears to be coming from where penetration lines designated SJ2, SJ3, WF and WS enter the annulus.

Remote visual tank wall inspection revealed no areas of concern since last evaluated on 3/5/05.

Remote visual tank wall inspection revealed no areas of concern since last evaluated on 3/5/05.

Remote visual tank wall inspection revealed no areas of concern since last evaluated on $3 / 5 / 05$. Stains on the annulus floor were caused by the inleakage of water.

Remote visual inspection of the secondary vessel wall revealed no areas of concern since last evaluated on $2 / 10 / 05$.

Remote visual tank wall inspection revealed no areas of concern since last evaluated on $3 / 5 / 05$.

Remote visual tank wall inspection revealed no areas of concern since last evaluated on 3/5/05.

Remote visual tank wall inspection revealed no areas of concern since last evaluated on 3/5/05. last evaluated on 1/24/06. 


\begin{tabular}{|c|c|c|c|c|c|c|}
\hline \multirow{2}{*}{$\begin{array}{c}\text { AREA } \\
\mathrm{H}\end{array}$} & \multirow{2}{*}{$\begin{array}{c}\begin{array}{c}\text { TANK OR } \\
\text { ANCILLARY }\end{array} \\
40\end{array}$} & $\frac{\text { ACCESS OPENING }}{(\text { (A OR I) }}$ & \multirow{2}{*}{$\begin{array}{c}\text { DATE } \\
01 / 24 / 06\end{array}$} & \multicolumn{3}{|c|}{$\begin{array}{l}\text { INSPECTION METHOD } \\
\text { IDENTIFICATION NUMBER }\end{array}$} \\
\hline & & $\mathrm{P}-09 \quad(\mathrm{~A})$ & & WAP & I & P06006:06 \\
\hline $\mathrm{H}$ & 40 & $\mathrm{P}-10 \quad(\mathrm{~A})$ & $01 / 24 / 06$ & WAP & I & P06006:07 \\
\hline $\mathrm{H}$ & 40 & $\mathrm{P}-11 \quad(\mathrm{~A})$ & 07/15/06 & DP & 1 & P06220:01-27 \\
\hline $\mathrm{H}$ & 40 & $\mathrm{P}-12 \quad(\mathrm{~A})$ & $01 / 24 / 06$ & WAP & I & P06006:09 \\
\hline $\mathrm{H}$ & 40 & $\mathrm{P}-12 \quad(\mathrm{~A})$ & $03 / 20 / 06$ & UT & 1 & UT-06-006 \\
\hline
\end{tabular}




\begin{tabular}{|c|c|c|c|c|c|c|c|c|}
\hline \multirow{2}{*}{$\frac{\text { AREA }}{\mathrm{H}}$} & \multirow{2}{*}{$\begin{array}{c}\text { TANK OR } \\
\text { ANCILLARY } \\
41\end{array}$} & \multicolumn{2}{|c|}{$\frac{\text { ACCESS OPENING }}{(A \text { OR I) }}$} & \multirow{2}{*}{$\begin{array}{l}\text { DATE } \\
08 / 25 / 06\end{array}$} & \multicolumn{3}{|c|}{$\begin{array}{l}\text { INSPECTION METHOD } \\
\text { IDENTIFICATION NUMBER }\end{array}$} & \multirow{2}{*}{$\begin{array}{l}\quad \text { REMARKS } \\
\text { Remote visual tank wall inspection } \\
\text { revealed no areas of concern since } \\
\text { last evaluated on } 1 / 24 / 06 \text {. The } \\
\text { annulus jet beneath the F riser } \\
\text { showed no degradation per T-DS-G } \\
-00043 \text {. }\end{array}$} \\
\hline & & A-01 & (A) & & DP & l & P06243:01-27 & \\
\hline $\mathrm{H}$ & 41 & A-02 & (A) & $08 / 25 / 06$ & DP & l & P06250:21-23 & $\begin{array}{l}\text { The conductivity probe was properly } \\
\text { positioned per J-JX-G-0001. }\end{array}$ \\
\hline $\mathrm{H}$ & 41 & A-02 & $(\mathrm{A})$ & $08 / 25 / 06$ & DP & l & P06250:01-27 & $\begin{array}{l}\text { Remote visual tank wall inspection } \\
\text { revealed no areas of concern since } \\
\text { last evaluated on } 8 / 21 / 05 \text {. }\end{array}$ \\
\hline $\mathrm{H}$ & 41 & A-03 & (A) & $08 / 25 / 06$ & DP & l & P06251:01-27 & $\begin{array}{l}\text { Remote visual tank wall inspection } \\
\text { revealed no areas of concern since } \\
\text { last evaluated on } 8 / 21 / 05 \text {. }\end{array}$ \\
\hline $\mathrm{H}$ & 41 & A-03 & (A) & 08/25/06 & DP & l & P06251:22 & $\begin{array}{l}\text { The conductivity probe was properly } \\
\text { positioned per J-JX-G-0001. }\end{array}$ \\
\hline $\mathrm{H}$ & 41 & A-04 & (A) & 08/25/06 & DP & l & P06252:01-27 & $\begin{array}{l}\text { Remote visual tank wall inspection } \\
\text { revealed no areas of concern since } \\
\text { last evaluated on } 8 / 21 / 05 \text {. }\end{array}$ \\
\hline $\mathrm{H}$ & 41 & A- 04 & (A) & $08 / 25 / 06$ & $\mathrm{DP}$ & l & P06252:22 & $\begin{array}{l}\text { The conductivity probe was properly } \\
\text { positioned per J-JX-G-0001. }\end{array}$ \\
\hline $\mathrm{H}$ & 41 & P-01 & (A) & $01 / 24 / 06$ & WAP & l & P06008:14 & $\begin{array}{l}\text { Remote visual tank wall inspection } \\
\text { revealed no areas of concern since } \\
\text { last evaluated on } 8 / 21 / 05 \text {. The } \\
\text { annulus jet beneath the F riser } \\
\text { showed no degradation per T-DS-G } \\
-00043 \text {. }\end{array}$ \\
\hline $\mathrm{H}$ & 41 & P-02 & (A) & $01 / 24 / 06$ & WAP & I & P06008:15 & $\begin{array}{l}\text { Remote visual tank wall inspection } \\
\text { revealed no areas of concern since } \\
\text { last evaluated on } 8 / 21 / 05 \text {. }\end{array}$ \\
\hline $\mathrm{H}$ & 41 & P-03 & (A) & $01 / 24 / 06$ & WAP & l & P06008:01 & $\begin{array}{l}\text { Remote visual tank wall inspection } \\
\text { revealed no areas of concern since } \\
\text { last evaluated on } 8 / 21 / 05 \text {. }\end{array}$ \\
\hline $\mathrm{H}$ & 41 & P-04 & (A) & $01 / 24 / 06$ & WAP & I & P06008:02 & $\begin{array}{l}\text { Remote visual tank wall inspection } \\
\text { revealed no areas of concern since } \\
\text { last evaluated on } 8 / 21 / 05 \text {. }\end{array}$ \\
\hline $\mathrm{H}$ & 41 & P-05 & (A) & $01 / 24 / 06$ & WAP & I & P06008:03 & $\begin{array}{l}\text { Remote visual tank wall inspection } \\
\text { revealed no areas of concern since } \\
\text { last evaluated on } 8 / 21 / 05 \text {. }\end{array}$ \\
\hline
\end{tabular}




\begin{tabular}{|c|c|c|c|c|}
\hline AREA & $\begin{array}{c}\text { TANK OR } \\
\text { ANCILLARY }\end{array}$ & $\frac{\text { ACCESS OPENING }}{(A \text { OR I) }}$ & DATE & $\begin{array}{l}\text { INSPECTION METHOD } \\
\text { IDENTIFICATION NUMBER }\end{array}$ \\
\hline $\mathrm{H}$ & 41 & P-05 (A) & 04/19/06 & UT-06-007 \\
\hline
\end{tabular}

$\mathrm{H}$

$\mathrm{H}$

$\mathrm{H}$

$\mathrm{H}$

$\mathrm{H}$

$\mathrm{H}$

$\mathrm{H}$

$\mathrm{H}$

$\mathrm{H}$
41

41

P-06 (A)

P-07 (A)

P-08 (A)

P-09 (A)

P-10 (A)

$01 / 24 / 06$

41

P-11 (A)

$01 / 24 / 06$

P-12 (A)

01/24/06

41

$\mathrm{P}-13 \quad(\mathrm{~A})$

$01 / 24 / 06$

P-14 (A)

$01 / 24 / 06$
WAP

WAP

I

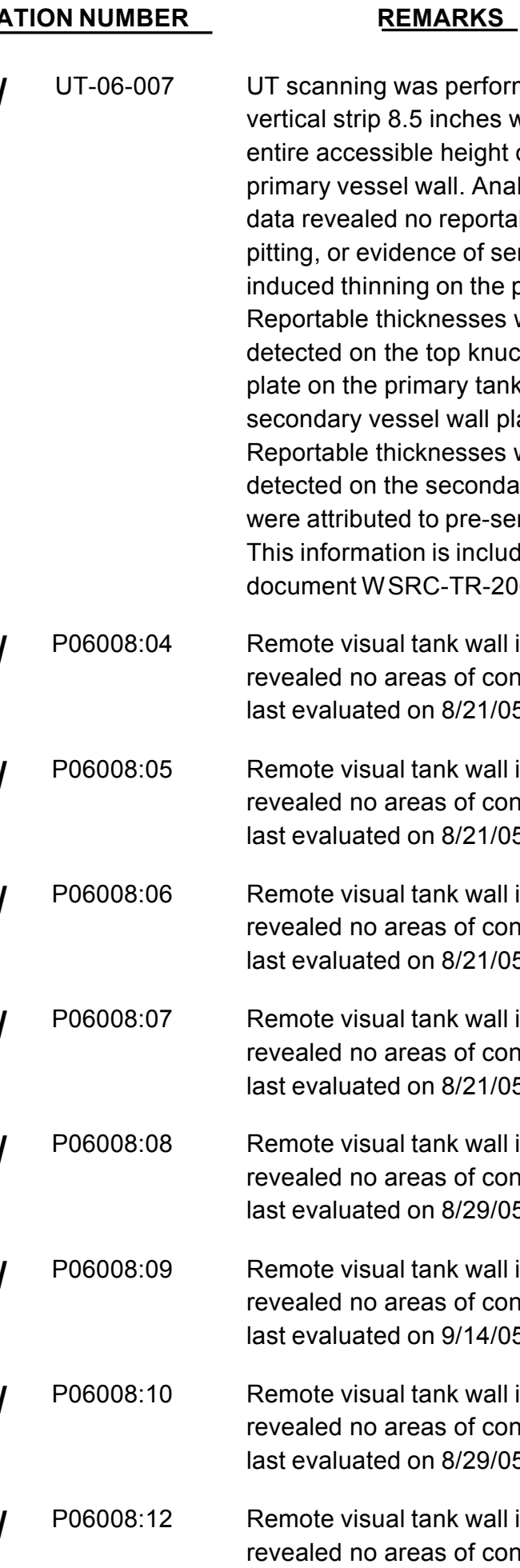

WAP

I

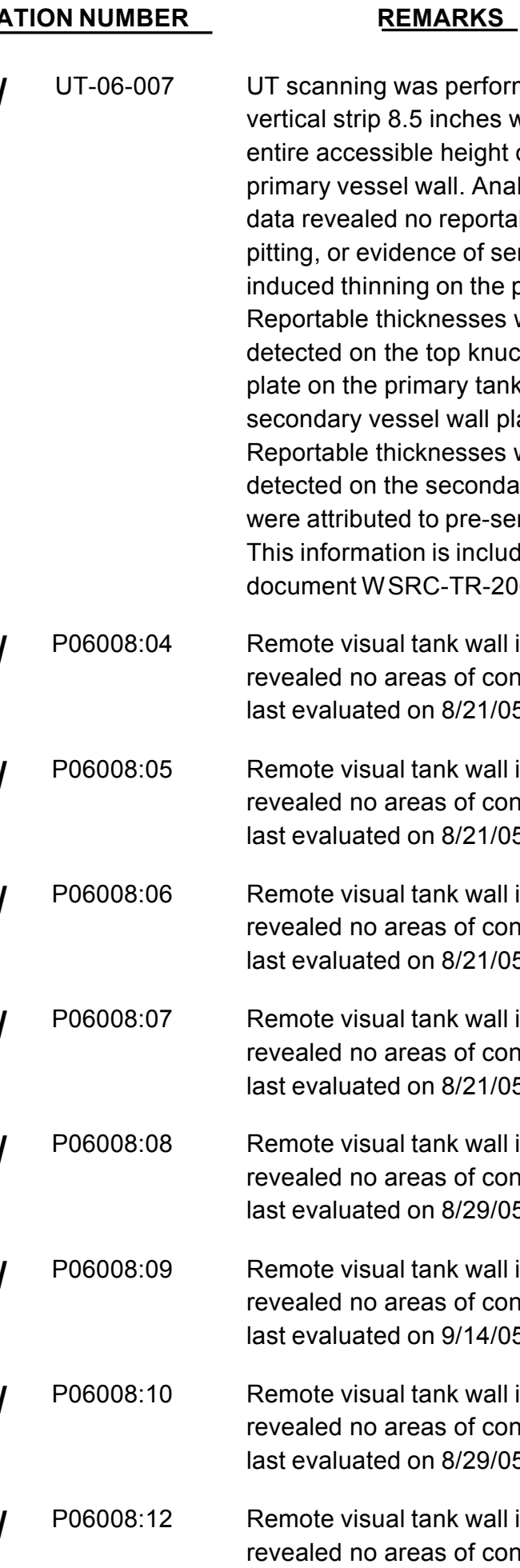

WAP

I

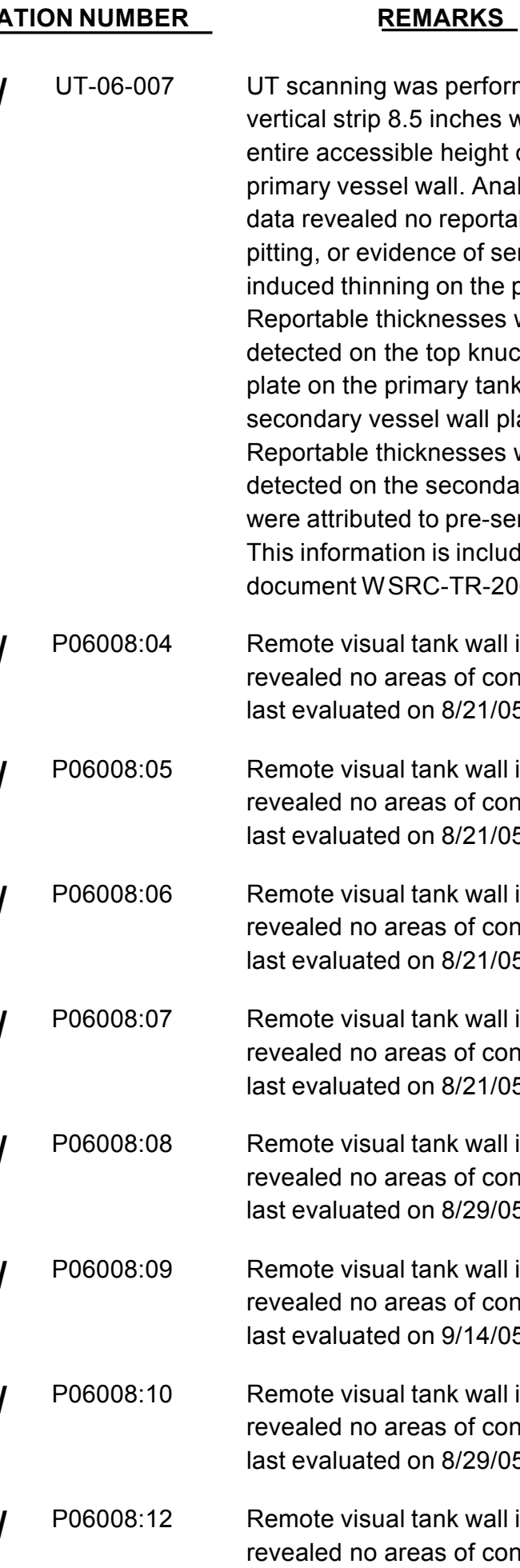

WAP

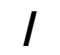

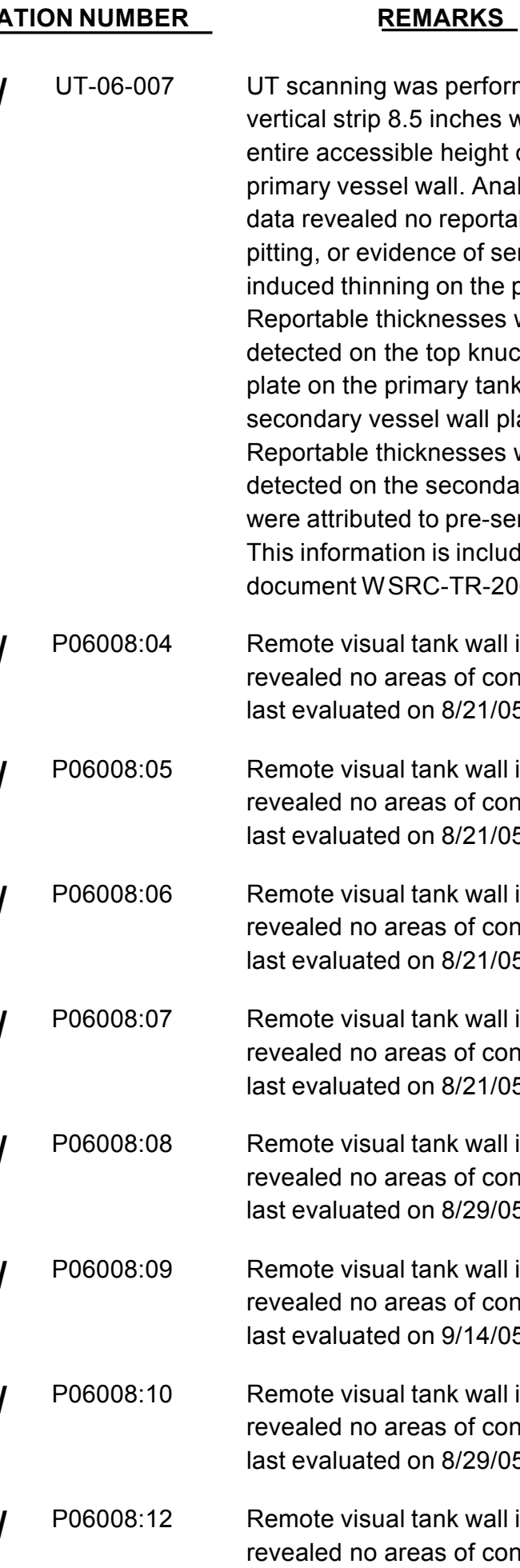

WAP

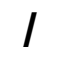

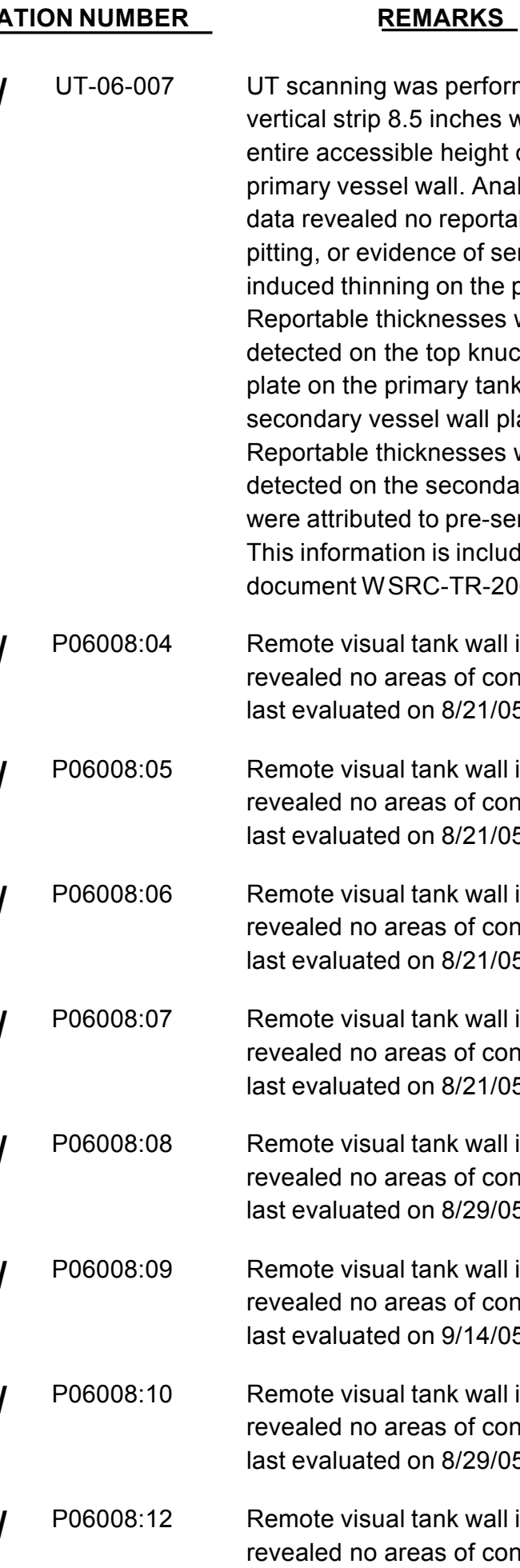

WAP

I

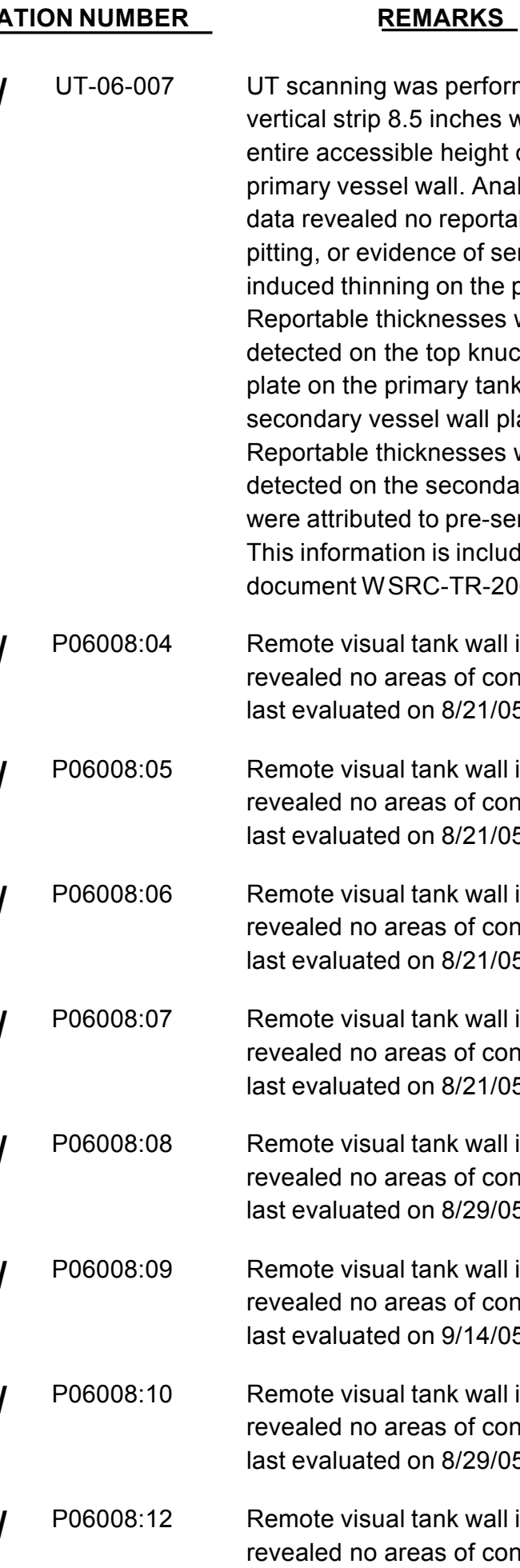

WAP

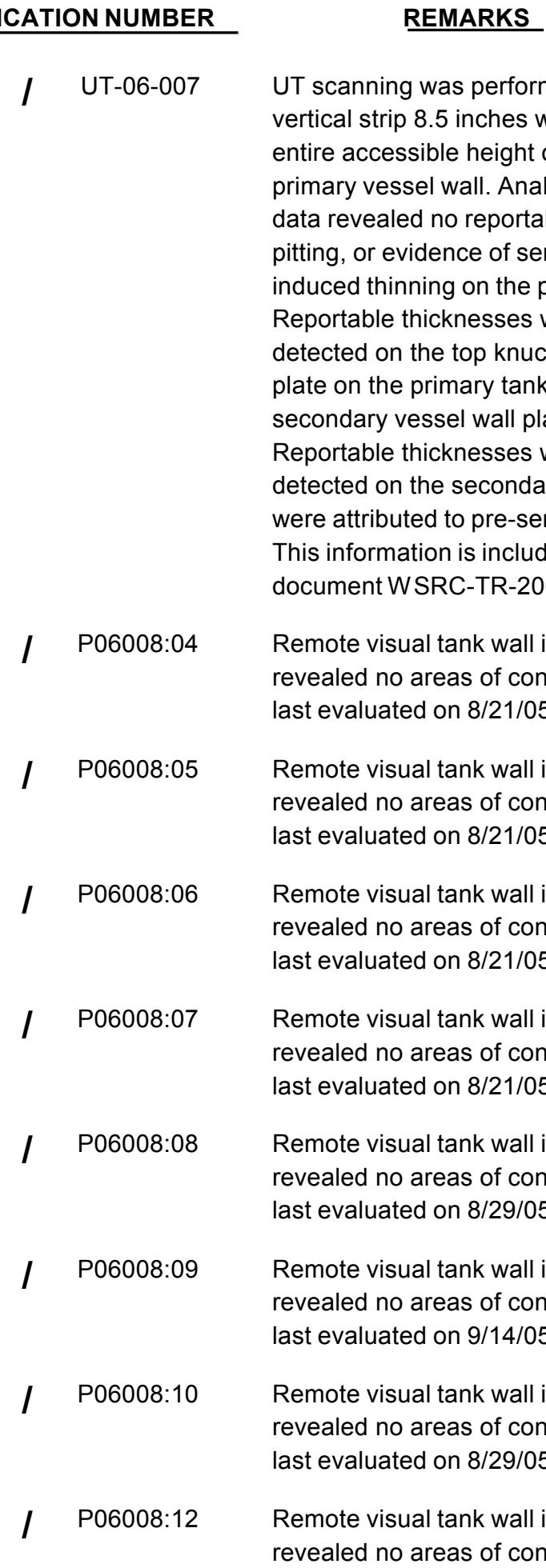

WAP

UT scanning was performed on a vertical strip 8.5 inches wide for the entire accessible height of the primary vessel wall. Analysis of the data revealed no reportable cracking, pitting, or evidence of serviceinning on the primary wall. were uckle and secondary vessel wall plate. Reportable thicknesses were also detected on the secondary floor but were attributed to pre-service pitting. This information is included in document WSRC-TR-2006-00002.

Remote visual tank wall inspection revealed no areas of concern since last evaluated on 8/21/05.

mote visual tank wall inspection revealed no areas of concern since Remote visual tank wall inspection revealed no areas of concern since

Remote visual tank wall inspection revealed no areas of concern since

Remote visual tank wall inspection revealed no areas of concern since evaluated on 8/29/05. concern since revealed no areas of concern last evaluated on 9/14/05.

Remote visual tank wall inspection revealed no areas of concern since last evaluated on 8/29/05. 


\begin{tabular}{|c|c|c|c|c|c|c|c|}
\hline \multirow{2}{*}{$\begin{array}{l}\text { AREA } \\
\mathrm{H}\end{array}$} & \multirow{2}{*}{$\begin{array}{c}\begin{array}{c}\text { TANK OR } \\
\text { ANCILLARY }\end{array} \\
42\end{array}$} & $\begin{array}{c}\text { ACCESS OPENING } \\
\text { (A OR I) }\end{array}$ & \multirow{2}{*}{$\frac{\text { DATE }}{01 / 24 / 06}$} & \multicolumn{3}{|c|}{$\begin{array}{l}\text { INSPECTION METHOD } \\
\text { IDENTIFICATION NUMBER }\end{array}$} & \multirow{2}{*}{$\begin{array}{l}\text { REMARKS } \\
\text { Remote visual tank wall inspection } \\
\text { revealed no areas of concern since } \\
\text { last evaluated on } 2 / 11 / 05 \text {. }\end{array}$} \\
\hline & & $A-01 \quad(A)$ & & WAP & l & P06011:07 & \\
\hline $\mathrm{H}$ & 42 & $\mathrm{~A}-02 \quad(\mathrm{~A})$ & $01 / 24 / 06$ & WAP & l & P06011:10 & $\begin{array}{l}\text { Remote visual tank wall inspection } \\
\text { revealed no areas of concern since } \\
\text { last evaluated on } 2 / 11 / 05 \text {. }\end{array}$ \\
\hline $\mathrm{H}$ & 42 & $\mathrm{~A}-03 \quad(\mathrm{~A})$ & $01 / 24 / 06$ & WAP & l & P06011:13 & $\begin{array}{l}\text { Remote visual tank wall inspection } \\
\text { revealed no areas of concern since } \\
\text { last evaluated on } 2 / 11 / 05 \text {. }\end{array}$ \\
\hline $\mathrm{H}$ & 42 & $\mathrm{~A}-04 \quad(\mathrm{~A})$ & $01 / 24 / 06$ & WAP & I & P06011:03 & $\begin{array}{l}\text { Remote visual tank wall inspection } \\
\text { revealed no areas of concern since } \\
\text { last evaluated on } 2 / 11 / 05 \text {. }\end{array}$ \\
\hline $\mathrm{H}$ & 42 & $\mathrm{P}-01 \quad(\mathrm{~A})$ & $01 / 24 / 06$ & WAP & I & P06011:08 & $\begin{array}{l}\text { Remote visual tank wall inspection } \\
\text { revealed no areas of concern since } \\
\text { last evaluated on } 3 / 5 / 05 \text {. The } \\
\text { annulus jet beneath the F riser } \\
\text { showed no degradation per T-DS-G } \\
-00043 \text {. }\end{array}$ \\
\hline $\mathrm{H}$ & 42 & $\mathrm{P}-02 \quad(\mathrm{~A})$ & $01 / 24 / 06$ & WAP & l & P06011:09 & $\begin{array}{l}\text { Remote visual tank wall inspection } \\
\text { revealed no areas of concern since } \\
\text { last evaluated on } 3 / 5 / 05 \text {. }\end{array}$ \\
\hline $\mathrm{H}$ & 42 & $\mathrm{P}-03 \quad(\mathrm{~A})$ & 07/15/06 & DP & I & P06213:01-26 & $\begin{array}{l}\text { Remote visual tank wall inspection } \\
\text { revealed no areas of concern since } \\
\text { last evaluated on } 2 / 10 / 05 \text {. }\end{array}$ \\
\hline $\mathrm{H}$ & 42 & $\mathrm{P}-03 \quad(\mathrm{~A})$ & $07 / 15 / 06$ & $\mathrm{DP}$ & I & P06214:01-27 & $\begin{array}{l}\text { Remote visual inspection of the } \\
\text { secondary vessel wall revealed no } \\
\text { areas of concern since last evaluated } \\
\text { on } 11 / 16 / 05 \text {. }\end{array}$ \\
\hline $\mathrm{H}$ & 42 & $\mathrm{P}-04 \quad(\mathrm{~A})$ & $01 / 24 / 06$ & WAP & I & P06011:11 & $\begin{array}{l}\text { Remote visual tank wall inspection } \\
\text { revealed no areas of concern since } \\
\text { last evaluated on } 3 / 5 / 05 \text {. }\end{array}$ \\
\hline $\mathrm{H}$ & 42 & P-05 (A) & $01 / 24 / 06$ & WAP & I & P06011:12 & $\begin{array}{l}\text { Remote visual tank wall inspection } \\
\text { revealed no areas of concern since } \\
\text { last evaluated on } 3 / 5 / 05 \text {. }\end{array}$ \\
\hline $\mathrm{H}$ & 42 & $\mathrm{P}-06 \quad(\mathrm{~A})$ & 09/20/06 & DP & I & P06270:01-27 & $\begin{array}{l}\text { Remote visual tank wall inspection } \\
\text { revealed no areas of concern since } \\
\text { last evaluated on } 3 / 5 / 05 \text {. }\end{array}$ \\
\hline $\mathrm{H}$ & 42 & $\mathrm{P}-07 \quad(\mathrm{~A})$ & 07/15/06 & DP & I & P06215:01-28 & $\begin{array}{l}\text { Remote visual tank wall inspection } \\
\text { revealed no areas of concern since } \\
\text { last evaluated on } 3 / 5 / 05 \text {. }\end{array}$ \\
\hline $\mathrm{H}$ & 42 & $\mathrm{P}-08 \quad(\mathrm{~A})$ & $01 / 24 / 06$ & WAP & I & P06011:14 & $\begin{array}{l}\text { Remote visual tank wall inspection } \\
\text { revealed no areas of concern since } \\
\text { last evaluated on } 3 / 5 / 05 \text {. }\end{array}$ \\
\hline
\end{tabular}




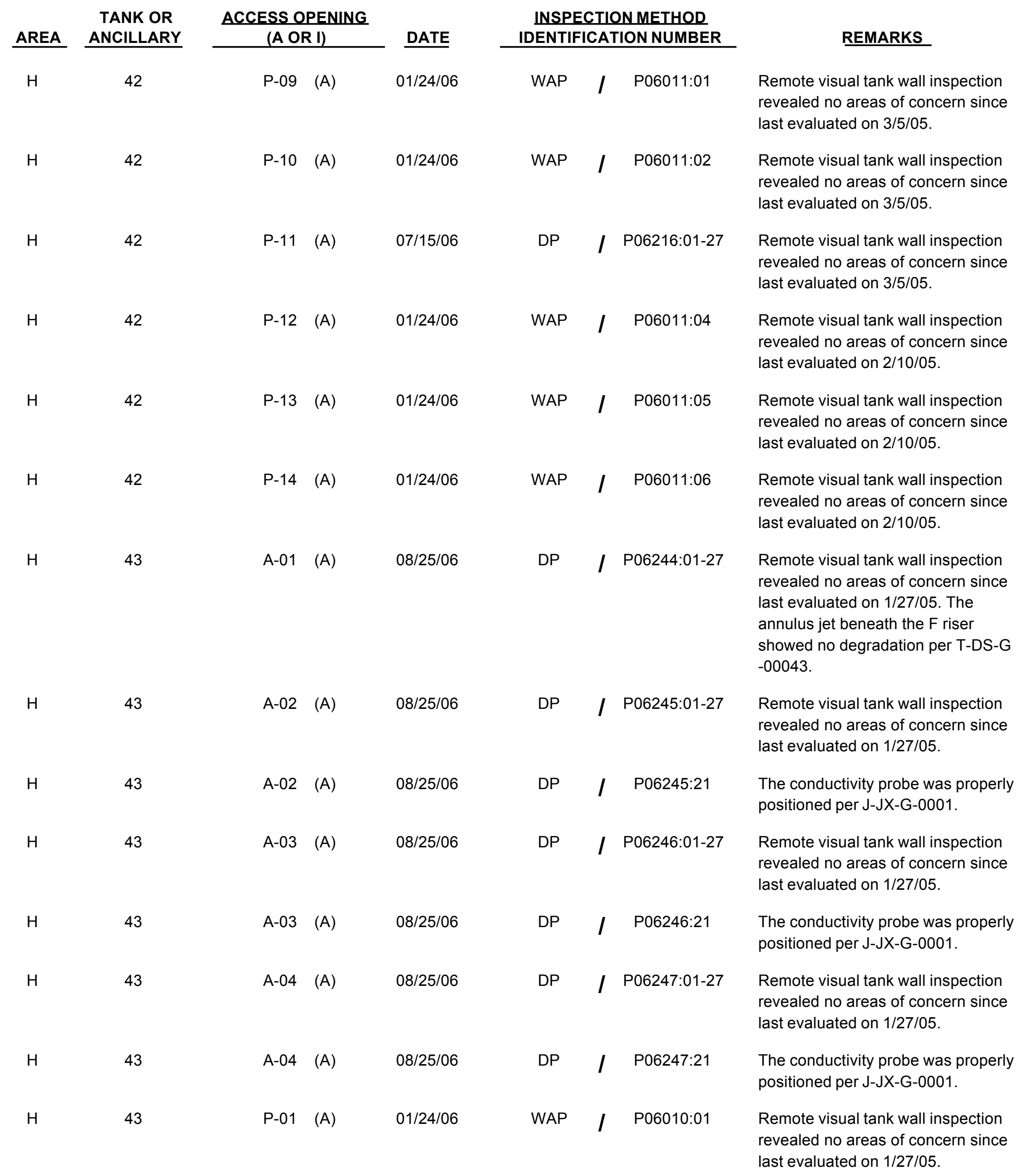




\begin{tabular}{|c|c|c|c|c|c|c|c|c|}
\hline \multirow{2}{*}{ AREA } & \multirow{2}{*}{$\begin{array}{c}\begin{array}{c}\text { TANK OR } \\
\text { ANCILLARY }\end{array} \\
43\end{array}$} & \multicolumn{2}{|c|}{$\frac{\text { ACCESS OPENING }}{\text { (A OR I) }}$} & \multirow{2}{*}{$\begin{array}{l}\text { DATE } \\
01 / 24 / 06\end{array}$} & \multicolumn{3}{|c|}{$\begin{array}{l}\text { INSPECTION METHOD } \\
\text { IDENTIFICATION NUMBER }\end{array}$} & \multirow{2}{*}{$\begin{array}{l}\text { REMARKS } \\
\text { Remote visual tank wall inspection } \\
\text { revealed no areas of concern since } \\
\text { last evaluated on } 1 / 27 / 05 \text {. }\end{array}$} \\
\hline & & P-02 & (A) & & WAP & I & P06010:02 & \\
\hline $\mathrm{H}$ & 43 & P-03 & (A) & 01/24/06 & WAP & I & P06010:03 & $\begin{array}{l}\text { Remote visual tank wall inspection } \\
\text { revealed no areas of concern since } \\
\text { last evaluated on } 1 / 27 / 05 \text {. }\end{array}$ \\
\hline $\mathrm{H}$ & 43 & P-04 & (A) & $01 / 24 / 06$ & WAP & I & P06010:04 & $\begin{array}{l}\text { Remote visual tank wall inspection } \\
\text { revealed no areas of concern since } \\
\text { last evaluated on } 1 / 27 / 05 \text {. }\end{array}$ \\
\hline $\mathrm{H}$ & 43 & P-05 & (A) & $01 / 24 / 06$ & WAP & I & P06010:05 & $\begin{array}{l}\text { Remote visual tank wall inspection } \\
\text { revealed no areas of concern since } \\
\text { last evaluated on } 1 / 27 / 05 \text {. }\end{array}$ \\
\hline $\mathrm{H}$ & 43 & P-06 & (A) & $01 / 24 / 06$ & WAP & I & P06010:06 & $\begin{array}{l}\text { Remote visual tank wall inspection } \\
\text { revealed no areas of concern since } \\
\text { last evaluated on } 1 / 27 / 05 \text {. }\end{array}$ \\
\hline $\mathrm{H}$ & 43 & P-07 & (A) & 01/24/06 & WAP & I & P06010:07 & $\begin{array}{l}\text { Remote visual tank wall inspection } \\
\text { revealed no areas of concern since } \\
\text { last evaluated on } 1 / 27 / 05 \text {. }\end{array}$ \\
\hline $\mathrm{H}$ & 43 & P-08 & (A) & $01 / 24 / 06$ & WAP & I & P06010:08 & $\begin{array}{l}\text { Remote visual tank wall inspection } \\
\text { revealed no areas of concern since } \\
\text { last evaluated on } 1 / 27 / 05 \text {. }\end{array}$ \\
\hline $\mathrm{H}$ & 43 & P-09 & (A) & 01/24/06 & WAP & I & P06010:14 & $\begin{array}{l}\text { Remote visual tank wall inspection } \\
\text { revealed no areas of concern since } \\
\text { last evaluated on } 1 / 27 / 05 \text {. }\end{array}$ \\
\hline $\mathrm{H}$ & 43 & P-10 & (A) & $01 / 24 / 06$ & WAP & I & P06010:13 & $\begin{array}{l}\text { Remote visual tank wall inspection } \\
\text { revealed no areas of concern since } \\
\text { last evaluated on } 2 / 9 / 05 \text {. }\end{array}$ \\
\hline $\mathrm{H}$ & 43 & P-11 & (A) & 01/24/06 & WAP & I & P06010:09 & $\begin{array}{l}\text { Remote visual tank wall inspection } \\
\text { revealed no areas of concern since } \\
\text { last evaluated on } 2 / 9 / 05 \text {. }\end{array}$ \\
\hline $\mathrm{H}$ & 43 & P-12 & (A) & $01 / 24 / 06$ & WAP & I & P06010:10 & $\begin{array}{l}\text { Remote visual tank wall inspection } \\
\text { revealed no areas of concern since } \\
\text { last evaluated on } 2 / 9 / 05 \text {. }\end{array}$ \\
\hline
\end{tabular}




\begin{tabular}{|c|c|c|c|c|}
\hline AREA & $\begin{array}{c}\text { TANK OR } \\
\text { ANCILLARY }\end{array}$ & $\frac{\text { ACCESS OPENING }}{(A \text { OR I) }}$ & DATE & $\begin{array}{l}\text { INSPECTION METHOD } \\
\text { IDENTIFICATION NUMBER }\end{array}$ \\
\hline $\mathrm{H}$ & 43 & $\mathrm{P}-12 \quad(\mathrm{~A})$ & $05 / 03 / 06$ & UT-06-008 \\
\hline
\end{tabular}

P-13 (A)

P-14 (A)

COP \#30

A-01 (A)

A-02 (A)

03/19/06

A-02 (A)

12/05/06

A-03 (A)

05/15/06

01/24/06

$01 / 24 / 06$

05/25/06

03/19/06
WAP

I

P06044:01

WAP

I

P06044:06

CTV

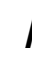

1520

WAP

P06087:02
P06010:11

P06010:12

1395
4

A-03 (A)

A-03 (A) 12/10/06

\section{REMARKS}

UT scanning was performed on a vertical strip 8.5 inches wide for the entire accessible height of the primary vessel wall. Reportable thicknesses were detected in the top and middle plates of the primary vessel, but are attributed to fabrication artifacts. Reportable thicknesses were detected in two of four secondary wall plates and the annulus floor. Analysis of the data revealed no reportable cracking, or other reportable thinning or pitting in the primary or secondary wall.

Grinding areas were observed in all plates. This information is included in document WSRC-TR-2006-00002.

Remote visual tank wall inspection revealed no areas of concern since last evaluated on 2/9/05.

Remote visual tank wall inspection revealed no areas of concern since last evaluated on 2/9/05.

Inspection of the transfer lines within the clean out port revealed no degradation per T-DS-G-00001.

Remote visual tank wall inspection revealed no areas of concern since last evaluated on 6/1/05.

Remote visual tank wall inspection revealed no areas of concern since last evaluated on 6/1/05.

The conductivity probe was properly deployed per J-JX-G-0001.

Remote visual tank wall inspection revealed no areas of concern since last evaluated on $6 / 1 / 05$. The annulus jet beneath the $\mathrm{F}$ riser showed no degradation per T-DS-G -00043 .

The conductivity probe was properly deployed per J-JX-G-0001. 


\begin{tabular}{|c|c|c|c|c|c|c|c|c|}
\hline \multirow{2}{*}{$\frac{\text { AREA }}{F}$} & \multirow{2}{*}{$\begin{array}{c}\text { TANK OR } \\
\text { ANCILLARY } \\
44\end{array}$} & \multicolumn{2}{|c|}{$\frac{\text { ACCESS OPENING }}{(A \text { OR I) }}$} & \multirow{2}{*}{$\begin{array}{l}\text { DATE } \\
03 / 19 / 06\end{array}$} & \multicolumn{3}{|c|}{$\begin{array}{l}\text { INSPECTION METHOD } \\
\text { IDENTIFICATION NUMBER }\end{array}$} & \multirow{2}{*}{$\begin{array}{l}\quad \text { REMARKS } \\
\text { Remote visual tank wall inspection } \\
\text { revealed no areas of concern since } \\
\text { last evaluated on } 9 / 4 / 05 \text {. Stains and } \\
\text { deposits on the secondary vessel } \\
\text { wall were caused by the inleakage of } \\
\text { water. }\end{array}$} \\
\hline & & A-04 & (A) & & WAP & l & P06044:12 & \\
\hline $\mathrm{F}$ & 44 & A-04 & (A) & $12 / 04 / 06$ & CCTV & l & 1520 & $\begin{array}{l}\text { The conductivity probe was properly } \\
\text { deployed per J-JX-G-0001. }\end{array}$ \\
\hline $\mathrm{F}$ & 44 & P-01 & (A) & $05 / 19 / 06$ & $\mathrm{DP}$ & I & P06134:01-28 & $\begin{array}{l}\text { Remote visual tank wall inspection } \\
\text { revealed no areas of concern since } \\
\text { last evaluated on } 5 / 16 / 05 \text {. }\end{array}$ \\
\hline $\mathrm{F}$ & 44 & P-01 & (A) & 05/19/06 & DP & I & P06135:01-26 & $\begin{array}{l}\text { Remote visual inspection of the } \\
\text { secondary vessel wall revealed no } \\
\text { areas of concern since last evaluated } \\
\text { on } 5 / 16 / 05 \text {. }\end{array}$ \\
\hline $\mathrm{F}$ & 44 & P-02 & (A) & 05/19/06 & $\mathrm{DP}$ & I & P06136:01-27 & $\begin{array}{l}\text { Remote visual tank wall inspection } \\
\text { revealed no areas of concern since } \\
\text { last evaluated on } 5 / 16 / 05 \text {. Stains } \\
\text { and marks on the secondary vessel } \\
\text { wall, ventilation duct, and annulus } \\
\text { floor were caused by the inleakage } \\
\text { of water. }\end{array}$ \\
\hline $\mathrm{F}$ & 44 & P-03 & (A) & 03/19/06 & WAP & I & P06044:02 & $\begin{array}{l}\text { Remote visual tank wall inspection } \\
\text { revealed no areas of concern since } \\
\text { last evaluated on } 5 / 16 / 05 \text {. Stains } \\
\text { and marks observed on the top of } \\
\text { the ventilation duct and annulus floor } \\
\text { were caused by the inleakage of } \\
\text { water. The inleakage originates from } \\
\text { where penetration line designated } \\
\text { SP enters the annulus. }\end{array}$ \\
\hline $\mathrm{F}$ & 44 & P-04 & (A) & 03/19/06 & WAP & I & P06044:03 & $\begin{array}{l}\text { Remote visual tank wall inspection } \\
\text { revealed no areas of concern since } \\
\text { last evaluated on } 5 / 16 / 05 \text {. Stains } \\
\text { and marks observed on the top of } \\
\text { the ventilation duct and annulus floor } \\
\text { were caused by the inleakage of } \\
\text { water. The inleakage originates from } \\
\text { where penetration line designated } \\
\text { SP enters the annulus. }\end{array}$ \\
\hline $\mathrm{F}$ & 44 & P-05 & (A) & 03/19/06 & WAP & I & P06044:04 & $\begin{array}{l}\text { Remote visual tank wall inspection } \\
\text { revealed no areas of concern since } \\
\text { last evaluated on } 5 / 16 / 05 \text {. }\end{array}$ \\
\hline $\mathrm{F}$ & 44 & P-06 & (A) & 03/19/06 & WAP & I & P06044:05 & $\begin{array}{l}\text { Remote visual tank wall inspection } \\
\text { revealed no areas of concern since } \\
\text { last evaluated on } 5 / 16 / 05 \text {. }\end{array}$ \\
\hline
\end{tabular}




\begin{tabular}{|c|c|c|c|c|c|c|c|c|}
\hline \multirow{2}{*}{ AREA } & \multirow{2}{*}{$\begin{array}{c}\text { TANK OR } \\
\text { ANCILLARY } \\
44\end{array}$} & \multicolumn{2}{|c|}{$\begin{array}{c}\text { ACCESS OPENING } \\
\text { (A OR I) }\end{array}$} & \multirow{2}{*}{$\begin{array}{c}\text { DATE } \\
03 / 19 / 06\end{array}$} & \multicolumn{3}{|c|}{$\begin{array}{l}\text { INSPECTION METHOD } \\
\text { IDENTIFICATION NUMBER }\end{array}$} & \multirow{2}{*}{$\begin{array}{l}\text { REMARKS } \\
\text { Remote visual tank wall inspection } \\
\text { revealed no areas of concern since } \\
\text { last evaluated on } 5 / 16 / 05 \text {. }\end{array}$} \\
\hline & & P-07 & (A) & & WAP & I & P06044:07 & \\
\hline $\mathrm{F}$ & 44 & P-08 & (A) & 05/19/06 & DP & I & P06137:01-27 & $\begin{array}{l}\text { Remote visual tank wall inspection } \\
\text { revealed no areas of concern since } \\
\text { last evaluated on } 9 / 3 / 05 \text {. }\end{array}$ \\
\hline $\mathrm{F}$ & 44 & P-09 & (A) & 04/27/06 & CCTV & l & 1255 & $\begin{array}{l}\text { CCTV was used to determine } \\
\text { location of water inleakage. No wet } \\
\text { areas were observed. However, } \\
\text { evidence of water inleakage was } \\
\text { observed where penetration line } \\
\text { designated SJ1 enters the annulus } \\
\text { and where the annulus cover plate } \\
\text { rests on top of the secondary liner. }\end{array}$ \\
\hline $\mathrm{F}$ & 44 & P-09 & (A) & 05/19/06 & DP & I & P06138:01-27 & $\begin{array}{l}\text { Remote visual tank wall inspection } \\
\text { revealed no areas of concern since } \\
\text { last evaluated on } 5 / 16 / 05 \text {. The } \\
\text { annulus jet beneath the F riser } \\
\text { showed no degradation per T-DS-G } \\
-00043 \text {. }\end{array}$ \\
\hline $\mathrm{F}$ & 44 & P-10 & (A) & 05/15/06 & WAP & I & P06087:01 & $\begin{array}{l}\text { Remote visual tank wall inspection } \\
\text { revealed no areas of concern since } \\
\text { last evaluated on } 9 / 3 / 05 \text {. }\end{array}$ \\
\hline $\mathrm{F}$ & 44 & P-11 & (A) & 03/19/06 & WAP & I & P06044:10 & $\begin{array}{l}\text { Remote visual tank wall inspection } \\
\text { revealed no areas of concern since } \\
\text { last evaluated on } 5 / 16 / 05 \text {. }\end{array}$ \\
\hline $\mathrm{F}$ & 44 & P-12 & (A) & 03/19/06 & WAP & I & P06044:11 & $\begin{array}{l}\text { Remote visual tank wall inspection } \\
\text { revealed no areas of concern since } \\
\text { last evaluated on } 5 / 16 / 05 \text {. }\end{array}$ \\
\hline $\mathrm{F}$ & 44 & P-13 & (A) & 03/19/06 & WAP & I & P06044:13 & $\begin{array}{l}\text { Remote visual tank wall inspection } \\
\text { revealed no areas of concern since } \\
\text { last evaluated on } 5 / 16 / 05 \text {. }\end{array}$ \\
\hline $\mathrm{F}$ & 44 & P-14 & (A) & 03/19/06 & WAP & I & P06044:14 & $\begin{array}{l}\text { Remote visual tank wall inspection } \\
\text { revealed no areas of concern since } \\
\text { last evaluated on } 5 / 16 / 05 \text {. }\end{array}$ \\
\hline $\mathrm{F}$ & 45 & A-01 & (A) & 03/19/06 & WAP & I & P06046:01 & $\begin{array}{l}\text { Remote visual tank wall inspection } \\
\text { revealed no areas of concern since } \\
\text { last evaluated on } 6 / 2 / 05 \text {. }\end{array}$ \\
\hline $\mathrm{F}$ & 45 & A-02 & (A) & 03/19/06 & WAP & I & P06046:06 & $\begin{array}{l}\text { Remote visual tank wall inspection } \\
\text { revealed no areas of concern since } \\
\text { last evaluated on } 6 / 2 / 05 \text {. }\end{array}$ \\
\hline
\end{tabular}




\begin{tabular}{|c|c|c|c|c|c|c|c|}
\hline \multirow{2}{*}{$\begin{array}{l}\text { AREA } \\
\mathrm{F}\end{array}$} & \multirow{2}{*}{$\begin{array}{c}\text { TANK OR } \\
\text { ANCILLARY } \\
45\end{array}$} & $\frac{\text { ACCESS OPENING }}{(A \text { OR I) }}$ & \multirow{2}{*}{$\begin{array}{l}\text { DATE } \\
03 / 19 / 06\end{array}$} & \multicolumn{3}{|c|}{$\begin{array}{l}\text { INSPECTION METHOD } \\
\text { IDENTIFICATION NUMBER }\end{array}$} & \multirow{2}{*}{$\begin{array}{l}\quad \text { REMARKS } \\
\text { Remote visual tank wall inspection } \\
\text { revealed no areas of concern since } \\
\text { last evaluated on } 6 / 2 / 05 \text {. The } \\
\text { annulus jet beneath the } F \text { riser } \\
\text { showed no degradation per T-DS-G } \\
-00043 \text {. }\end{array}$} \\
\hline & & $A-03 \quad(A)$ & & WAP & l & P06046:08 & \\
\hline $\mathrm{F}$ & 45 & $A-04 \quad(A)$ & 03/19/06 & WAP & l & P06046:12 & $\begin{array}{l}\text { Remote visual tank wall inspection } \\
\text { revealed no areas of concern since } \\
\text { last evaluated on } 6 / 2 / 05 \text {. }\end{array}$ \\
\hline $\mathrm{F}$ & 45 & $\mathrm{P}-01 \quad(\mathrm{~A})$ & 05/19/06 & $\mathrm{DP}$ & I & P06152:01-27 & $\begin{array}{l}\text { Remote visual inspection of the } \\
\text { secondary vessel wall revealed no } \\
\text { areas of concern since last evaluated } \\
\text { on } 5 / 14 / 05 \text {. }\end{array}$ \\
\hline $\mathrm{F}$ & 45 & $\mathrm{P}-01 \quad(\mathrm{~A})$ & 05/19/06 & $\mathrm{DP}$ & l & P06151:01-26 & $\begin{array}{l}\text { Remote visual tank wall inspection } \\
\text { revealed no areas of concern since } \\
\text { last evaluated on } 5 / 16 / 05 \text {. }\end{array}$ \\
\hline $\mathrm{F}$ & 45 & $\mathrm{P}-02 \quad(\mathrm{~A})$ & 05/19/06 & $\mathrm{DP}$ & I & P06153:01-28 & $\begin{array}{l}\text { Remote visual tank wall inspection } \\
\text { revealed no areas of concern since } \\
\text { last evaluated on } 5 / 16 / 05 \text {. }\end{array}$ \\
\hline $\mathrm{F}$ & 45 & $\mathrm{P}-03 \quad(\mathrm{~A})$ & 03/19/06 & WAP & l & P06046:02 & $\begin{array}{l}\text { Remote visual tank wall inspection } \\
\text { revealed no areas of concern since } \\
\text { last evaluated on } 5 / 16 / 05 \text {. }\end{array}$ \\
\hline $\mathrm{F}$ & 45 & P-04 (A) & 03/19/06 & WAP & l & P06046:03 & $\begin{array}{l}\text { Remote visual tank wall inspection } \\
\text { revealed no areas of concern since } \\
\text { last evaluated on } 5 / 16 / 05 \text {. }\end{array}$ \\
\hline $\mathrm{F}$ & 45 & $\mathrm{P}-05 \quad(\mathrm{~A})$ & 03/19/06 & WAP & I & P06046:04 & $\begin{array}{l}\text { Remote visual tank wall inspection } \\
\text { revealed no areas of concern since } \\
\text { last evaluated on } 5 / 16 / 05 \text {. }\end{array}$ \\
\hline $\mathrm{F}$ & 45 & $\mathrm{P}-06 \quad(\mathrm{~A})$ & 03/19/06 & WAP & I & P06046:05 & $\begin{array}{l}\text { Remote visual tank wall inspection } \\
\text { revealed no areas of concern since } \\
\text { last evaluated on } 5 / 16 / 05 \text {. }\end{array}$ \\
\hline $\mathrm{F}$ & 45 & $\mathrm{P}-07 \quad(\mathrm{~A})$ & 03/19/06 & WAP & I & P06046:07 & $\begin{array}{l}\text { Remote visual tank wall inspection } \\
\text { revealed no areas of concern since } \\
\text { last evaluated on } 5 / 16 / 05 \text {. }\end{array}$ \\
\hline $\mathrm{F}$ & 45 & $\mathrm{P}-08 \quad(\mathrm{~A})$ & 05/19/06 & DP & I & P06154:01-27 & $\begin{array}{l}\text { Remote visual tank wall inspection } \\
\text { revealed no areas of concern since } \\
\text { last evaluated on } 5 / 16 / 05 \text {. }\end{array}$ \\
\hline $\mathrm{F}$ & 45 & P-09 (A) & 05/19/06 & $\mathrm{DP}$ & I & P06155:01-28 & $\begin{array}{l}\text { Remote visual tank wall inspection } \\
\text { revealed no areas of concern since } \\
\text { last evaluated on } 5 / 16 / 05 \text {. }\end{array}$ \\
\hline $\mathrm{F}$ & 45 & $\mathrm{P}-10 \quad(\mathrm{~A})$ & 03/19/06 & WAP & I & P06046:09 & $\begin{array}{l}\text { Remote visual tank wall inspection } \\
\text { revealed no areas of concern since } \\
\text { last evaluated on } 5 / 16 / 05 \text {. }\end{array}$ \\
\hline
\end{tabular}




\begin{tabular}{|c|c|c|c|c|c|c|c|c|}
\hline \multirow{2}{*}{$\frac{\text { AREA }}{\mathrm{F}}$} & \multirow{2}{*}{$\begin{array}{c}\text { TANK OR } \\
\text { ANCILLARY } \\
45\end{array}$} & \multicolumn{2}{|c|}{$\frac{\text { ACCESS OPENING }}{(\text { A OR I) }}$} & \multirow{2}{*}{$\frac{\text { DATE }}{03 / 19 / 06}$} & \multicolumn{3}{|c|}{$\begin{array}{l}\text { INSPECTION METHOD } \\
\text { IDENTIFICATION NUMBER }\end{array}$} & \multirow{2}{*}{$\begin{array}{l}\text { REMARKS } \\
\text { Remote visual tank wall inspection } \\
\text { revealed no areas of concern since } \\
\text { last evaluated on } 5 / 16 / 05 \text {. }\end{array}$} \\
\hline & & $\mathrm{P}-11$ & (A) & & WAP & l & P06046:10 & \\
\hline $\mathrm{F}$ & 45 & $\mathrm{P}-12$ & (A) & 03/19/06 & WAP & l & P06046:11 & $\begin{array}{l}\text { Remote visual tank wall inspection } \\
\text { revealed no areas of concern since } \\
\text { last evaluated on } 5 / 16 / 05 \text {. }\end{array}$ \\
\hline $\mathrm{F}$ & 45 & P-13 & (A) & 03/19/06 & WAP & l & P06046:13 & $\begin{array}{l}\text { Remote visual tank wall inspection } \\
\text { revealed no areas of concern since } \\
\text { last evaluated on } 5 / 16 / 05 \text {. }\end{array}$ \\
\hline $\mathrm{F}$ & 45 & $\mathrm{P}-14$ & (A) & 03/19/06 & WAP & l & P06046:14 & $\begin{array}{l}\text { Remote visual tank wall inspection } \\
\text { revealed no areas of concern since } \\
\text { last evaluated on } 9 / 3 / 05 \text {. }\end{array}$ \\
\hline $\mathrm{F}$ & 46 & $A-01$ & (A) & 05/13/06 & WAP & l & P06098:13 & $\begin{array}{l}\text { Remote visual tank wall inspection } \\
\text { revealed no areas of concern since } \\
\text { last evaluated on } 6 / 2 / 05 \text {. }\end{array}$ \\
\hline $\mathrm{F}$ & 46 & A-02 & (A) & 05/13/06 & WAP & l & P06098:09 & $\begin{array}{l}\text { Remote visual tank wall inspection } \\
\text { revealed no areas of concern since } \\
\text { last evaluated on } 6 / 2 / 05 \text {. }\end{array}$ \\
\hline $\mathrm{F}$ & 46 & A-02 & (A) & $12 / 11 / 06$ & CCTV & l & 1520 & $\begin{array}{l}\text { The conductivity probe was properly } \\
\text { deployed per J-JX-G-0001. }\end{array}$ \\
\hline $\mathrm{F}$ & 46 & A-03 & (A) & 05/13/06 & WAP & l & P06098:06 & $\begin{array}{l}\text { Remote visual tank wall inspection } \\
\text { revealed no areas of concern since } \\
\text { last evaluated on } 6 / 2 / 05 \text {. }\end{array}$ \\
\hline $\mathrm{F}$ & 46 & A-03 & (A) & $11 / 26 / 06$ & CCTV & l & 1499 & $\begin{array}{l}\text { The conductivity probe was properly } \\
\text { deployed per J-JX-G-0001. }\end{array}$ \\
\hline $\mathrm{F}$ & 46 & A-04 & (A) & 05/13/06 & WAP & I & P06098:02 & $\begin{array}{l}\text { Remote visual tank wall inspection } \\
\text { revealed no areas of concern since } \\
\text { last evaluated on } 6 / 2 / 05 \text {. }\end{array}$ \\
\hline $\mathrm{F}$ & 46 & A-04 & (A) & $11 / 26 / 06$ & CCTV & l & 1499 & $\begin{array}{l}\text { The conductivity probe was properly } \\
\text { deployed per J-JX-G-0001. }\end{array}$ \\
\hline $\mathrm{F}$ & 46 & $\mathrm{P}-01$ & (A) & 05/19/06 & DP & l & P06109:01-26 & $\begin{array}{l}\text { Remote visual tank wall inspection } \\
\text { revealed no areas of concern since } \\
\text { last evaluated on } 5 / 16 / 05 \text {. }\end{array}$ \\
\hline $\mathrm{F}$ & 46 & P-01 & (A) & 05/19/06 & DP & I & P06113:01-27 & $\begin{array}{l}\text { Remote visual inspection of the } \\
\text { secondary vessel wall revealed no } \\
\text { areas of concern since last evaluated } \\
\text { on } 9 / 4 / 05 \text {. }\end{array}$ \\
\hline $\mathrm{F}$ & 46 & P-02 & (A) & 05/19/06 & DP & l & P06110:01-26 & $\begin{array}{l}\text { Remote visual tank wall inspection } \\
\text { revealed no areas of concern since } \\
\text { last evaluated on } 5 / 16 / 05 \text {. }\end{array}$ \\
\hline
\end{tabular}




\begin{tabular}{|c|c|c|c|c|c|c|c|c|}
\hline \multirow{2}{*}{ AREA } & \multirow{2}{*}{$\begin{array}{c}\text { TANK OR } \\
\text { ANCILLARY } \\
46\end{array}$} & \multicolumn{2}{|c|}{$\begin{array}{c}\text { ACCESS OPENING } \\
\text { (A OR I) }\end{array}$} & \multirow{2}{*}{$\begin{array}{l}\text { DATE } \\
\text { 05/13/06 }\end{array}$} & \multicolumn{3}{|c|}{$\begin{array}{l}\text { INSPECTION METHOD } \\
\text { IDENTIFICATION NUMBER }\end{array}$} & \multirow{2}{*}{$\begin{array}{l}\text { REMARKS } \\
\text { Remote visual tank wall inspection } \\
\text { revealed no areas of concern since } \\
\text { last evaluated on } 5 / 16 / 05 \text {. }\end{array}$} \\
\hline & & P-03 & (A) & & WAP & l & P06098:01 & \\
\hline $\mathrm{F}$ & 46 & P-04 & (A) & 05/13/06 & WAP & l & P06098:03 & $\begin{array}{l}\text { Remote visual tank wall inspection } \\
\text { revealed no areas of concern since } \\
\text { last evaluated on } 9 / 3 / 05 \text {. }\end{array}$ \\
\hline $\mathrm{F}$ & 46 & P-05 & (A) & 05/13/06 & WAP & I & P06098:04 & $\begin{array}{l}\text { Remote visual tank wall inspection } \\
\text { revealed no areas of concern since } \\
\text { last evaluated on } 5 / 16 / 05 \text {. }\end{array}$ \\
\hline $\mathrm{F}$ & 46 & P-06 & (A) & 05/13/06 & WAP & l & P06098:05 & $\begin{array}{l}\text { Remote visual tank wall inspection } \\
\text { revealed no areas of concern since } \\
\text { last evaluated on } 5 / 16 / 05 \text {. }\end{array}$ \\
\hline $\mathrm{F}$ & 46 & P-07 & (A) & $05 / 13 / 06$ & WAP & l & P06098:07 & $\begin{array}{l}\text { Remote visual tank wall inspection } \\
\text { revealed no areas of concern since } \\
\text { last evaluated on } 5 / 16 / 05 \text {. The } \\
\text { annulus jet beneath the } F \text { riser } \\
\text { showed no degradation per T-DS-G } \\
-00043 \text {. }\end{array}$ \\
\hline $\mathrm{F}$ & 46 & P-08 & (A) & 05/19/06 & $\mathrm{DP}$ & I & P06111:01-27 & $\begin{array}{l}\text { Remote visual tank wall inspection } \\
\text { revealed no areas of concern since } \\
\text { last evaluated on } 5 / 16 / 05 \text {. }\end{array}$ \\
\hline $\mathrm{F}$ & 46 & P-09 & (A) & 05/19/06 & $\mathrm{DP}$ & l & P06112:01-27 & $\begin{array}{l}\text { Remote visual tank wall inspection } \\
\text { revealed no areas of concern since } \\
\text { last evaluated on } 5 / 16 / 05 \text {. }\end{array}$ \\
\hline $\mathrm{F}$ & 46 & $P-10$ & (A) & $05 / 13 / 06$ & WAP & I & P06098:08 & $\begin{array}{l}\text { Remote visual tank wall inspection } \\
\text { revealed no areas of concern since } \\
\text { last evaluated on } 5 / 16 / 05 \text {. }\end{array}$ \\
\hline $\mathrm{F}$ & 46 & P-11 & (A) & 05/13/06 & WAP & l & P06098:10 & $\begin{array}{l}\text { Remote visual tank wall inspection } \\
\text { revealed no areas of concern since } \\
\text { last evaluated on } 5 / 16 / 05 \text {. An } \\
\text { increase in stains and marks on the } \\
\text { secondary vessel wall was caused } \\
\text { by the inleakage of water. }\end{array}$ \\
\hline $\mathrm{F}$ & 46 & P-12 & (A) & 05/13/06 & WAP & l & P06098:11 & $\begin{array}{l}\text { Remote visual tank wall inspection } \\
\text { revealed no areas of concern since } \\
\text { last evaluated on } 5 / 16 / 05 \text {. }\end{array}$ \\
\hline $\mathrm{F}$ & 46 & P-13 & (A) & $05 / 13 / 06$ & WAP & l & P06098:12 & $\begin{array}{l}\text { Remote visual tank wall inspection } \\
\text { revealed no areas of concern since } \\
\text { last evaluated on } 5 / 16 / 05 \text {. }\end{array}$ \\
\hline $\mathrm{F}$ & 46 & P-14 & (A) & 07/18/06 & WAP & l & P06195:01 & $\begin{array}{l}\text { Remote visual tank wall inspection } \\
\text { revealed no areas of concern since } \\
\text { last evaluated on } 5 / 16 / 05 \text {. }\end{array}$ \\
\hline
\end{tabular}




\begin{tabular}{|c|c|c|c|c|c|c|c|c|}
\hline \multirow{2}{*}{ AREA } & \multirow{2}{*}{$\begin{array}{c}\text { TANK OR } \\
\text { ANCILLARY } \\
47\end{array}$} & \multicolumn{2}{|c|}{$\begin{array}{c}\text { ACCESS OPENING } \\
\text { (A OR I) }\end{array}$} & \multirow{2}{*}{$\frac{\text { DATE }}{05 / 25 / 06}$} & \multicolumn{3}{|c|}{$\begin{array}{l}\text { INSPECTION METHOD } \\
\text { IDENTIFICATION NUMBER }\end{array}$} & \multirow{2}{*}{$\begin{array}{l}\text { REMARKS } \\
\text { Inspection of the transfer lines within } \\
\text { the clean out port revealed no } \\
\text { degradation per T-DS-G-00001. }\end{array}$} \\
\hline & & COP \#31 & & & CCTV & l & 1396 & \\
\hline $\mathrm{F}$ & 47 & $A-01$ & (A) & 05/13/06 & WAP & l & P06099:11 & $\begin{array}{l}\text { Remote visual tank wall inspection } \\
\text { revealed no areas of concern since } \\
\text { last evaluated on } 6 / 2 / 05 \text {. }\end{array}$ \\
\hline $\mathrm{F}$ & 47 & $\mathrm{~A}-02$ & (A) & 05/13/06 & WAP & l & P06099:09 & $\begin{array}{l}\text { Remote visual tank wall inspection } \\
\text { revealed no areas of concern since } \\
\text { last evaluated on } 6 / 2 / 05 \text {. }\end{array}$ \\
\hline $\mathrm{F}$ & 47 & $\mathrm{~A}-02$ & (A) & $11 / 26 / 06$ & CCTV & l & 1499 & $\begin{array}{l}\text { The conductivity probe was properly } \\
\text { deployed per J-JX-G-0001. }\end{array}$ \\
\hline $\mathrm{F}$ & 47 & A-03 & (A) & 05/13/06 & WAP & l & P06099:06 & $\begin{array}{l}\text { Remote visual tank wall inspection } \\
\text { revealed no areas of concern since } \\
\text { last evaluated on } 6 / 2 / 05 \text {. }\end{array}$ \\
\hline $\mathrm{F}$ & 47 & $A-03$ & (A) & $12 / 04 / 06$ & CCTV & l & 1520 & $\begin{array}{l}\text { The conductivity probe was properly } \\
\text { deployed per J-JX-G-0001. }\end{array}$ \\
\hline $\mathrm{F}$ & 47 & A-04 & (A) & $05 / 13 / 06$ & WAP & l & P06099:01 & $\begin{array}{l}\text { Remote visual tank wall inspection } \\
\text { revealed no areas of concern since } \\
\text { last evaluated on } 6 / 2 / 05 \text {. }\end{array}$ \\
\hline $\mathrm{F}$ & 47 & A-04 & (A) & $11 / 26 / 06$ & CCTV & l & 1499 & $\begin{array}{l}\text { The conductivity probe was properly } \\
\text { deployed per J-JX-G-0001. }\end{array}$ \\
\hline $\mathrm{F}$ & 47 & P-01 & (A) & 05/19/06 & DP & I & P06120:01-27 & $\begin{array}{l}\text { Remote visual tank wall inspection } \\
\text { revealed no areas of concern since } \\
\text { last evaluated on } 5 / 16 / 05 \text {. }\end{array}$ \\
\hline $\mathrm{F}$ & 47 & P-01 & (A) & 05/19/06 & DP & I & P06121:01-27 & $\begin{array}{l}\text { Remote visual inspection of the } \\
\text { secondary vessel wall revealed no } \\
\text { areas of concern since last evaluated } \\
\text { on } 5 / 17 / 05 \text {. }\end{array}$ \\
\hline $\mathrm{F}$ & 47 & P-02 & (A) & 05/19/06 & DP & I & P06122:01-27 & $\begin{array}{l}\text { Remote visual tank wall inspection } \\
\text { revealed no areas of concern since } \\
\text { last evaluated on } 5 / 16 / 05 \text {. }\end{array}$ \\
\hline $\mathrm{F}$ & 47 & P-03 & (A) & $05 / 13 / 06$ & WAP & l & P06099:02 & $\begin{array}{l}\text { Remote visual tank wall inspection } \\
\text { revealed no areas of concern since } \\
\text { last evaluated on } 5 / 16 / 05 \text {. }\end{array}$ \\
\hline $\mathrm{F}$ & 47 & P-04 & (A) & 05/13/06 & WAP & l & P06099:03 & $\begin{array}{l}\text { Remote visual tank wall inspection } \\
\text { revealed no areas of concern since } \\
\text { last evaluated on } 5 / 16 / 05 \text {. }\end{array}$ \\
\hline $\mathrm{F}$ & 47 & P-05 & (A) & 05/13/06 & WAP & I & P06099:04 & $\begin{array}{l}\text { Remote visual tank wall inspection } \\
\text { revealed no areas of concern since } \\
\text { last evaluated on } 5 / 16 / 05 \text {. }\end{array}$ \\
\hline
\end{tabular}




\begin{tabular}{|c|c|c|c|c|c|c|c|c|}
\hline \multirow{2}{*}{ AREA } & \multirow{2}{*}{$\begin{array}{c}\text { TANK OR } \\
\text { ANCILLARY } \\
47\end{array}$} & \multicolumn{2}{|c|}{$\frac{\text { ACCESS OPENING }}{(A \text { OR I) }}$} & \multirow{2}{*}{$\frac{\text { DATE }}{05 / 13 / 06}$} & \multicolumn{3}{|c|}{$\begin{array}{l}\text { INSPECTION METHOD } \\
\text { IDENTIFICATION NUMBER }\end{array}$} & \multirow{2}{*}{$\begin{array}{l}\text { REMARKS } \\
\text { Remote visual tank wall inspection } \\
\text { revealed no areas of concern since } \\
\text { last evaluated on } 5 / 16 / 05 \text {. }\end{array}$} \\
\hline & & P-06 & (A) & & WAP & l & P06099:05 & \\
\hline $\mathrm{F}$ & 47 & P-07 & (A) & $05 / 13 / 06$ & WAP & I & P06099:07 & $\begin{array}{l}\text { Remote visual tank wall inspection } \\
\text { revealed no areas of concern since } \\
\text { last evaluated on } 5 / 16 / 05 \text {. The } \\
\text { annulus jet beneath the F riser } \\
\text { showed no degradation per T-DS-G } \\
-00043 \text {. }\end{array}$ \\
\hline $\mathrm{F}$ & 47 & P-08 & (A) & 05/19/06 & $\mathrm{DP}$ & I & P06123:01-27 & $\begin{array}{l}\text { Remote visual tank wall inspection } \\
\text { revealed no areas of concern since } \\
\text { last evaluated on } 5 / 16 / 05 \text {. }\end{array}$ \\
\hline $\mathrm{F}$ & 47 & P-09 & (A) & 07/25/06 & DP & I & P06225:01-26 & $\begin{array}{l}\text { Remote visual tank wall inspection } \\
\text { revealed no areas of concern since } \\
\text { last evaluated on } 5 / 16 / 05 \text {. }\end{array}$ \\
\hline $\mathrm{F}$ & 47 & $P-10$ & (A) & $05 / 13 / 06$ & WAP & I & P06099:08 & $\begin{array}{l}\text { Remote visual tank wall inspection } \\
\text { revealed no areas of concern since } \\
\text { last evaluated on } 5 / 16 / 05 \text {. }\end{array}$ \\
\hline $\mathrm{F}$ & 47 & P-11 & (A) & 05/13/06 & WAP & l & P06099:10 & $\begin{array}{l}\text { Remote visual tank wall inspection } \\
\text { revealed no areas of concern since } \\
\text { last evaluated on } 5 / 16 / 05 \text {. }\end{array}$ \\
\hline $\mathrm{F}$ & 47 & P-12 & (A) & $05 / 13 / 06$ & WAP & I & P06099:12 & $\begin{array}{l}\text { Remote visual tank wall inspection } \\
\text { revealed no areas of concern since } \\
\text { last evaluated on } 5 / 16 / 05 \text {. }\end{array}$ \\
\hline $\mathrm{F}$ & 47 & $P-13$ & (A) & 05/13/06 & WAP & l & P06099:13 & $\begin{array}{l}\text { Remote visual tank wall inspection } \\
\text { revealed no areas of concern since } \\
\text { last evaluated on } 5 / 16 / 05 \text {. }\end{array}$ \\
\hline $\mathrm{F}$ & 47 & P-14 & (A) & $05 / 13 / 06$ & WAP & I & P06099:14 & $\begin{array}{l}\text { Remote visual tank wall inspection } \\
\text { revealed no areas of concern since } \\
\text { last evaluated on } 5 / 16 / 05 \text {. }\end{array}$ \\
\hline $\mathrm{H}$ & 48 & $A-01$ & (A) & $04 / 26 / 06$ & WAP & I & P06054:10 & $\begin{array}{l}\text { Remote visual tank wall inspection } \\
\text { revealed no areas of concern since } \\
\text { last evaluated on } 6 / 29 / 05 \text {. }\end{array}$ \\
\hline $\mathrm{H}$ & 48 & A-02 & (A) & 04/26/06 & WAP & I & P06054:02 & $\begin{array}{l}\text { Remote visual tank wall inspection } \\
\text { revealed no areas of concern since } \\
\text { last evaluated on } 12 / 13 / 05 \text {. }\end{array}$ \\
\hline $\mathrm{H}$ & 48 & A-03 & (A) & $04 / 26 / 06$ & WAP & I & P06054:04 & $\begin{array}{l}\text { Remote visual tank wall inspection } \\
\text { revealed no areas of concern since } \\
\text { last evaluated on } 6 / 22 / 05 \text {. }\end{array}$ \\
\hline $\mathrm{H}$ & 48 & A-04 & (A) & $04 / 26 / 06$ & WAP & I & P06054:07 & $\begin{array}{l}\text { Remote visual tank wall inspection } \\
\text { revealed no areas of concern since } \\
\text { last evaluated on } 6 / 22 / 05 \text {. }\end{array}$ \\
\hline
\end{tabular}




\begin{tabular}{|c|c|c|c|c|c|c|c|c|}
\hline \multirow{2}{*}{$\frac{\text { AREA }}{\mathrm{H}}$} & \multirow{2}{*}{$\begin{array}{c}\text { TANK OR } \\
\text { ANCILLARY } \\
48\end{array}$} & \multicolumn{2}{|c|}{$\frac{\text { ACCESS OPENING }}{\text { (A OR I) }}$} & \multirow{2}{*}{$\begin{array}{l}\text { DATE } \\
05 / 11 / 06\end{array}$} & \multicolumn{3}{|c|}{$\begin{array}{l}\text { INSPECTION METHOD } \\
\text { IDENTIFICATION NUMBER }\end{array}$} & \multirow[b]{2}{*}{$\begin{array}{l}\text { REMARKS } \\
\text { Remote visual tank wall inspection } \\
\text { revealed no areas of concern since } \\
\text { last evaluated on } 6 / 29 / 05 \text {. Stains } \\
\text { and marks on the secondary vessel } \\
\text { wall were caused by the inleakage of } \\
\text { water. The annulus jet beneath the } \\
\text { F riser showed no degradation per T. } \\
\text { DS-G-00043. }\end{array}$} \\
\hline & & P-01 & (A) & & $\mathrm{DP}$ & I & P06064:01-28 & \\
\hline $\mathrm{H}$ & 48 & P-02 & (A) & 05/10/06 & $\mathrm{DP}$ & I & P06057:01-27 & $\begin{array}{l}\text { Remote visual tank wall inspection } \\
\text { revealed no areas of concern since } \\
\text { last evaluated on } 6 / 29 / 05 \text {. }\end{array}$ \\
\hline $\mathrm{H}$ & 48 & P-03 & (A) & $04 / 26 / 06$ & WAP & I & P06054:11 & $\begin{array}{l}\text { Remote visual tank wall inspection } \\
\text { revealed no areas of concern since } \\
\text { last evaluated on } 6 / 17 / 05 \text {. }\end{array}$ \\
\hline $\mathrm{H}$ & 48 & P-04 & (A) & $04 / 26 / 06$ & WAP & I & P06054:01 & $\begin{array}{l}\text { Remote visual tank wall inspection } \\
\text { revealed no areas of concern since } \\
\text { last evaluated on } 6 / 17 / 05 \text {. }\end{array}$ \\
\hline $\mathrm{H}$ & 48 & P-05 & (A) & $04 / 26 / 06$ & WAP & I & P06054:03 & $\begin{array}{l}\text { Remote visual tank wall inspection } \\
\text { revealed no areas of concern since } \\
\text { last evaluated on } 9 / 20 / 05 \text {. }\end{array}$ \\
\hline $\mathrm{H}$ & 48 & P-06 & (A) & 05/10/06 & $\mathrm{DP}$ & I & P06058:01-27 & $\begin{array}{l}\text { Remote visual tank wall inspection } \\
\text { revealed no areas of concern since } \\
\text { last evaluated on } 6 / 17 / 05 \text {. }\end{array}$ \\
\hline $\mathrm{H}$ & 48 & P-07 & (A) & 05/10/06 & $\mathrm{DP}$ & I & P06059:01-27 & $\begin{array}{l}\text { Remote visual tank wall inspection } \\
\text { revealed no areas of concern since } \\
\text { last evaluated on 6/29/05. }\end{array}$ \\
\hline $\mathrm{H}$ & 48 & P-07 & (A) & 05/10/06 & $\mathrm{DP}$ & I & P06063:01-27 & $\begin{array}{l}\text { Remote visual inspection of the } \\
\text { secondary vessel wall revealed no } \\
\text { areas of concern since last evaluated } \\
\text { on } 6 / 22 / 05 \text {. }\end{array}$ \\
\hline $\mathrm{H}$ & 48 & P-08 & (A) & 05/10/06 & $\mathrm{DP}$ & I & P06060:01-27 & $\begin{array}{l}\text { Remote visual tank wall inspection } \\
\text { revealed no areas of concern since } \\
\text { last evaluated on } 6 / 17 / 05 \text {. Stains on } \\
\text { the bottom knuckle plate were } \\
\text { caused by the inleakage of water. }\end{array}$ \\
\hline $\mathrm{H}$ & 48 & P-08 & (A) & $05 / 10 / 06$ & $\mathrm{DP}$ & I & P06060:20 & $\begin{array}{l}\text { The conductivity probe beneath the } \\
\text { A- } 03 \text { riser was properly positioned } \\
\text { per J-JX-G-0001. }\end{array}$ \\
\hline $\mathrm{H}$ & 48 & P-09 & (A) & 05/10/06 & $\mathrm{DP}$ & I & P06061:01-27 & $\begin{array}{l}\text { Remote visual tank wall inspection } \\
\text { revealed no areas of concern since } \\
\text { last evaluated on } 6 / 29 / 05 \text {. }\end{array}$ \\
\hline $\mathrm{H}$ & 48 & P-10 & (A) & $04 / 26 / 06$ & WAP & I & P06054:05 & $\begin{array}{l}\text { Remote visual tank wall inspection } \\
\text { revealed no areas of concern since } \\
\text { last evaluated on } 6 / 17 / 05 \text {. }\end{array}$ \\
\hline
\end{tabular}




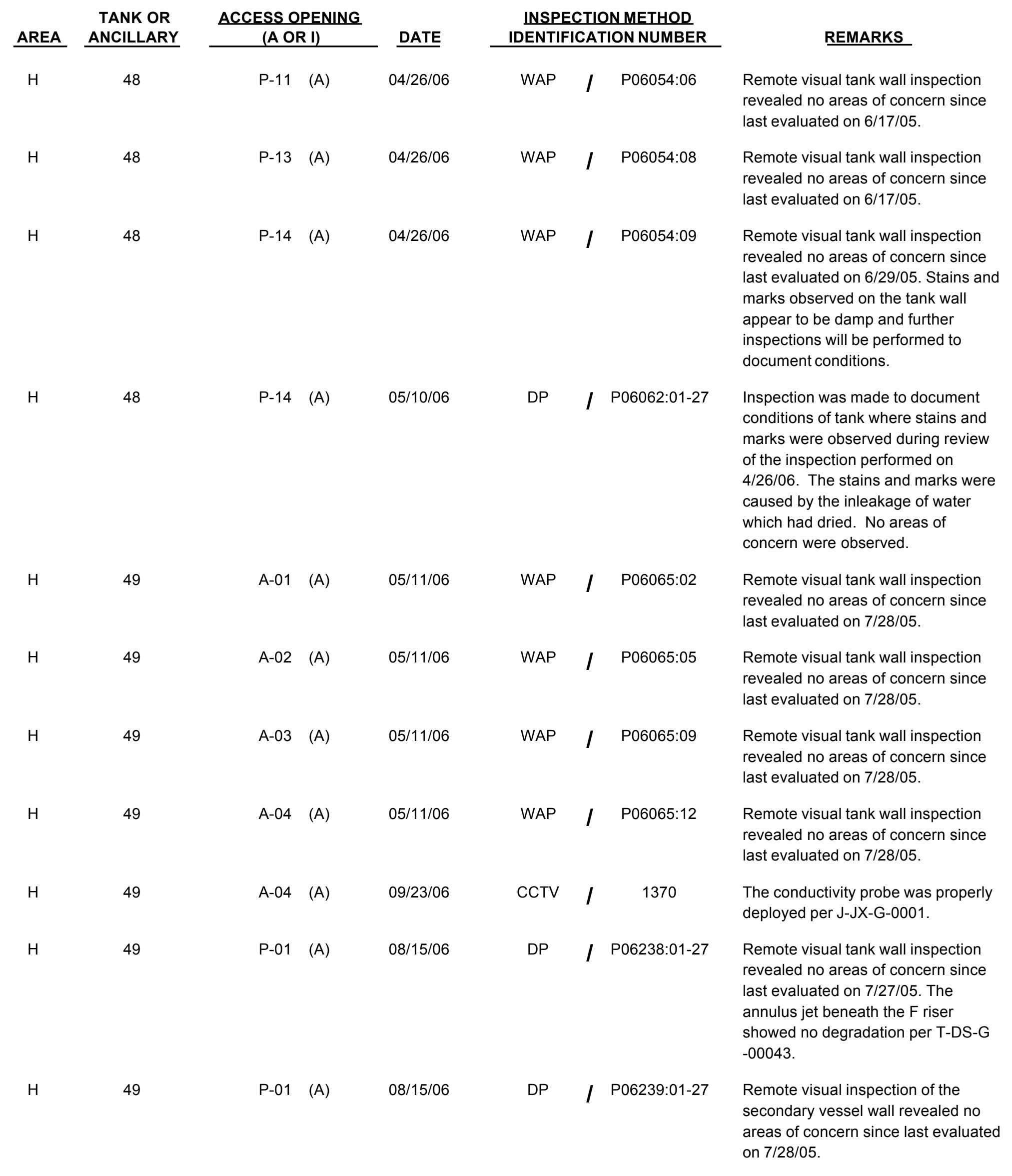




\begin{tabular}{|c|c|c|c|c|c|c|c|c|}
\hline \multirow{2}{*}{ AREA } & \multirow{2}{*}{$\begin{array}{c}\text { TANK OR } \\
\text { ANCILLARY } \\
49\end{array}$} & \multicolumn{2}{|c|}{$\begin{array}{c}\text { ACCESS OPENING } \\
\text { (A OR I) }\end{array}$} & \multirow{2}{*}{$\begin{array}{c}\text { DATE } \\
08 / 15 / 06\end{array}$} & \multicolumn{3}{|c|}{$\begin{array}{l}\text { INSPECTION METHOD } \\
\text { IDENTIFICATION NUMBER }\end{array}$} & \multirow{2}{*}{$\begin{array}{l}\text { REMARKS } \\
\text { Remote visual tank wall inspection } \\
\text { revealed no areas of concern since } \\
\text { last evaluated on } 7 / 27 / 05 \text {. }\end{array}$} \\
\hline & & P-02 & (A) & & $\mathrm{DP}$ & I & P06240:01-27 & \\
\hline $\mathrm{H}$ & 49 & P-03 & (A) & $05 / 11 / 06$ & WAP & I & P06065:03 & $\begin{array}{l}\text { Remote visual tank wall inspection } \\
\text { revealed no areas of concern since } \\
\text { last evaluated on } 7 / 27 / 05 \text {. }\end{array}$ \\
\hline $\mathrm{H}$ & 49 & $\mathrm{P}-04$ & (A) & 05/11/06 & WAP & l & P06065:04 & $\begin{array}{l}\text { Remote visual tank wall inspection } \\
\text { revealed no areas of concern since } \\
\text { last evaluated on } 7 / 27 / 05 \text {. }\end{array}$ \\
\hline $\mathrm{H}$ & 49 & P-05 & (A) & $05 / 11 / 06$ & WAP & l & P06065:06 & $\begin{array}{l}\text { Remote visual tank wall inspection } \\
\text { revealed no areas of concern since } \\
\text { last evaluated on } 7 / 27 / 05 \text {. }\end{array}$ \\
\hline $\mathrm{H}$ & 49 & P-06 & (A) & $05 / 11 / 06$ & WAP & I & P06065:07 & $\begin{array}{l}\text { Remote visual tank wall inspection } \\
\text { revealed no areas of concern since } \\
\text { last evaluated on } 7 / 27 / 05 \text {. }\end{array}$ \\
\hline $\mathrm{H}$ & 49 & P-07 & (A) & $05 / 11 / 06$ & WAP & l & P06065:08 & $\begin{array}{l}\text { Remote visual tank wall inspection } \\
\text { revealed no areas of concern since } \\
\text { last evaluated on } 7 / 27 / 05 \text {. An } \\
\text { increase in stains and marks } \\
\text { observed on the primary vessel wall } \\
\text { were caused by the inleakage of } \\
\text { water. }\end{array}$ \\
\hline $\mathrm{H}$ & 49 & P-08 & (A) & 08/15/06 & $\mathrm{DP}$ & I & P06241:01-27 & $\begin{array}{l}\text { Remote visual tank wall inspection } \\
\text { revealed no areas of concern since } \\
\text { last evaluated on } 7 / 27 / 05 \text {. }\end{array}$ \\
\hline $\mathrm{H}$ & 49 & P-09 & (A) & 08/15/06 & DP & I & P06242:01-27 & $\begin{array}{l}\text { Remote visual tank wall inspection } \\
\text { revealed no areas of concern since } \\
\text { last evaluated on } 7 / 27 / 05 \text {. }\end{array}$ \\
\hline $\mathrm{H}$ & 49 & $P-10$ & (A) & $05 / 11 / 06$ & WAP & I & P06065:10 & $\begin{array}{l}\text { Remote visual tank wall inspection } \\
\text { revealed no areas of concern since } \\
\text { last evaluated on } 7 / 27 / 05 \text {. }\end{array}$ \\
\hline $\mathrm{H}$ & 49 & $\mathrm{P}-11$ & (A) & 05/11/06 & WAP & I & P06065:11 & $\begin{array}{l}\text { Remote visual tank wall inspection } \\
\text { revealed no areas of concern since } \\
\text { last evaluated on } 7 / 27 / 05 \text {. }\end{array}$ \\
\hline $\mathrm{H}$ & 49 & $\mathrm{P}-12$ & (A) & 05/11/06 & WAP & I & P06065:14 & $\begin{array}{l}\text { Remote visual tank wall inspection } \\
\text { revealed no areas of concern since } \\
\text { last evaluated on } 7 / 27 / 05 \text {. }\end{array}$ \\
\hline $\mathrm{H}$ & 49 & P-13 & (A) & $05 / 11 / 06$ & WAP & I & P06065:13 & $\begin{array}{l}\text { Remote visual tank wall inspection } \\
\text { revealed no areas of concern since } \\
\text { last evaluated on } 7 / 27 / 05 \text {. }\end{array}$ \\
\hline $\mathrm{H}$ & 49 & P-14 & (A) & $05 / 11 / 06$ & WAP & I & P06065:01 & $\begin{array}{l}\text { Remote visual tank wall inspection } \\
\text { revealed no areas of concern since } \\
\text { last evaluated on } 7 / 27 / 05 \text {. }\end{array}$ \\
\hline
\end{tabular}




\begin{tabular}{|c|c|c|c|c|c|c|c|}
\hline \multirow{2}{*}{$\begin{array}{l}\text { AREA } \\
\mathrm{H}\end{array}$} & \multirow{2}{*}{$\begin{array}{c}\text { TANK OR } \\
\text { ANCILLARY } \\
50\end{array}$} & $\begin{array}{c}\text { ACCESS OPENING } \\
\text { (A OR I) }\end{array}$ & \multirow{2}{*}{$\frac{\text { DATE }}{05 / 11 / 06}$} & \multicolumn{3}{|c|}{$\begin{array}{l}\text { INSPECTION METHOD } \\
\text { IDENTIFICATION NUMBER }\end{array}$} & \multirow{2}{*}{$\begin{array}{l}\text { REMARKS } \\
\text { Remote visual tank wall inspection } \\
\text { revealed no areas of concern since } \\
\text { last evaluated on } 7 / 28 / 05 \text {. }\end{array}$} \\
\hline & & $A-01 \quad(A)$ & & WAP & l & P06066:03 & \\
\hline $\mathrm{H}$ & 50 & $A-02 \quad(A)$ & 05/11/06 & WAP & I & P06066:02 & $\begin{array}{l}\text { Remote visual tank wall inspection } \\
\text { revealed no areas of concern since } \\
\text { last evaluated on } 7 / 28 / 05 \text {. }\end{array}$ \\
\hline $\mathrm{H}$ & 50 & $\mathrm{~A}-03 \quad(\mathrm{~A})$ & 05/11/06 & WAP & l & P06066:01 & $\begin{array}{l}\text { Remote visual tank wall inspection } \\
\text { revealed no areas of concern since } \\
\text { last evaluated on } 7 / 28 / 05 \text {. }\end{array}$ \\
\hline $\mathrm{H}$ & 50 & $\mathrm{~A}-04 \quad(\mathrm{~A})$ & 03/08/06 & WAP & I & P06038:10 & $\begin{array}{l}\text { Remote visual tank wall inspection } \\
\text { revealed no areas of concern since } \\
\text { last evaluated on } 7 / 28 / 05 \text {. }\end{array}$ \\
\hline $\mathrm{H}$ & 50 & $\mathrm{P}-01 \quad(\mathrm{~A})$ & 08/15/06 & $\mathrm{DP}$ & I & P06233:01-27 & $\begin{array}{l}\text { Remote visual inspection of the } \\
\text { secondary vessel wall revealed no } \\
\text { areas of concern since last evaluated } \\
\text { on } 7 / 30 / 05 \text {. }\end{array}$ \\
\hline $\mathrm{H}$ & 50 & $\mathrm{P}-01 \quad(\mathrm{~A})$ & 08/15/06 & $\mathrm{DP}$ & I & P06232:01-27 & $\begin{array}{l}\text { Remote visual tank wall inspection } \\
\text { revealed no areas of concern since } \\
\text { last evaluated on } 7 / 27 / 05 \text {. The } \\
\text { annulus jet beneath the F riser } \\
\text { showed no degradation per T-DS-G } \\
-00043 \text {. }\end{array}$ \\
\hline $\mathrm{H}$ & 50 & P-02 (A) & $08 / 15 / 06$ & $\mathrm{DP}$ & l & P06234:01-27 & $\begin{array}{l}\text { Remote visual tank wall inspection } \\
\text { revealed no areas of concern since } \\
\text { last evaluated on } 7 / 27 / 05 \text {. }\end{array}$ \\
\hline $\mathrm{H}$ & 50 & $\mathrm{P}-03 \quad(\mathrm{~A})$ & 03/08/06 & WAP & I & P06038:01 & $\begin{array}{l}\text { Remote visual tank wall inspection } \\
\text { revealed no areas of concern since } \\
\text { last evaluated on } 7 / 27 / 05 \text {. }\end{array}$ \\
\hline $\mathrm{H}$ & 50 & $\mathrm{P}-04 \quad(\mathrm{~A})$ & 03/08/06 & WAP & I & P06038:02 & $\begin{array}{l}\text { Remote visual tank wall inspection } \\
\text { revealed no areas of concern since } \\
\text { last evaluated on } 7 / 27 / 05 \text {. }\end{array}$ \\
\hline $\mathrm{H}$ & 50 & P-05 (A) & 03/08/06 & WAP & I & P06038:04 & $\begin{array}{l}\text { Remote visual tank wall inspection } \\
\text { revealed no areas of concern since } \\
\text { last evaluated on } 7 / 27 / 05 \text {. }\end{array}$ \\
\hline $\mathrm{H}$ & 50 & $\mathrm{P}-06 \quad(\mathrm{~A})$ & 03/08/06 & WAP & I & P06038:05 & $\begin{array}{l}\text { Remote visual tank wall inspection } \\
\text { revealed no areas of concern since } \\
\text { last evaluated on } 7 / 27 / 05 \text {. }\end{array}$ \\
\hline $\mathrm{H}$ & 50 & $\mathrm{P}-07 \quad(\mathrm{~A})$ & 03/08/06 & WAP & I & P06038:06 & $\begin{array}{l}\text { Remote visual tank wall inspection } \\
\text { revealed no areas of concern since } \\
\text { last evaluated on } 7 / 27 / 05 \text {. }\end{array}$ \\
\hline $\mathrm{H}$ & 50 & $\mathrm{P}-08 \quad(\mathrm{~A})$ & 08/15/06 & DP & I & P06235:01-27 & $\begin{array}{l}\text { Remote visual tank wall inspection } \\
\text { revealed no areas of concern since } \\
\text { last evaluated on } 7 / 27 / 05 \text {. }\end{array}$ \\
\hline
\end{tabular}




\begin{tabular}{|c|c|c|c|c|c|c|c|}
\hline \multirow{2}{*}{ AREA } & \multirow{2}{*}{$\begin{array}{c}\text { TANK OR } \\
\text { ANCILLARY } \\
50\end{array}$} & $\begin{array}{c}\text { ACCESS OPENING } \\
\text { (A OR I) }\end{array}$ & \multirow{2}{*}{$\frac{\text { DATE }}{08 / 15 / 06}$} & \multicolumn{3}{|c|}{$\begin{array}{l}\text { INSPECTION METHOD } \\
\text { IDENTIFICATION NUMBER }\end{array}$} & \multirow{2}{*}{$\begin{array}{l}\text { REMARKS } \\
\text { Remote visual tank wall inspection } \\
\text { revealed no areas of concern since } \\
\text { last evaluated on } 9 / 20 / 05 \text {. }\end{array}$} \\
\hline & & P-09 (A) & & $\mathrm{DP}$ & l & P06236:01-27 & \\
\hline $\mathrm{H}$ & 50 & $\mathrm{P}-10 \quad(\mathrm{~A})$ & 03/08/06 & WAP & I & P06038:08 & $\begin{array}{l}\text { Remote visual tank wall inspection } \\
\text { revealed no areas of concern since } \\
\text { last evaluated on } 7 / 27 / 05 \text {. }\end{array}$ \\
\hline $\mathrm{H}$ & 50 & $\mathrm{P}-11 \quad(\mathrm{~A})$ & 03/08/06 & WAP & l & P06038:09 & $\begin{array}{l}\text { Remote visual tank wall inspection } \\
\text { revealed no areas of concern since } \\
\text { last evaluated on } 7 / 27 / 05 \text {. }\end{array}$ \\
\hline $\mathrm{H}$ & 50 & $\mathrm{P}-12 \quad(\mathrm{~A})$ & 03/08/06 & WAP & I & P06038:11 & $\begin{array}{l}\text { Remote visual tank wall inspection } \\
\text { revealed no areas of concern since } \\
\text { last evaluated on } 7 / 27 / 05 \text {. }\end{array}$ \\
\hline $\mathrm{H}$ & 50 & $\mathrm{P}-13 \quad(\mathrm{~A})$ & 03/08/06 & WAP & l & P06038:12 & $\begin{array}{l}\text { Remote visual tank wall inspection } \\
\text { revealed no areas of concern since } \\
\text { last evaluated on } 7 / 27 / 05 \text {. }\end{array}$ \\
\hline $\mathrm{H}$ & 50 & $\mathrm{P}-14 \quad(\mathrm{~A})$ & $03 / 08 / 06$ & WAP & I & P06038:13 & $\begin{array}{l}\text { Remote visual tank wall inspection } \\
\text { revealed no areas of concern since } \\
\text { last evaluated on } 7 / 27 / 05 \text {. }\end{array}$ \\
\hline $\mathrm{H}$ & 51 & $A-01 \quad(A)$ & $03 / 08 / 06$ & WAP & l & P06037:06 & $\begin{array}{l}\text { Remote visual tank wall inspection } \\
\text { revealed no areas of concern since } \\
\text { last evaluated on } 7 / 28 / 05 \text {. }\end{array}$ \\
\hline $\mathrm{H}$ & 51 & $\mathrm{~A}-02 \quad(\mathrm{~A})$ & 03/08/06 & WAP & l & P06037:02 & $\begin{array}{l}\text { Remote visual tank wall inspection } \\
\text { revealed no areas of concern since } \\
\text { last evaluated on } 7 / 28 / 05 \text {. }\end{array}$ \\
\hline $\mathrm{H}$ & 51 & $\mathrm{~A}-03 \quad(\mathrm{~A})$ & 03/08/06 & WAP & I & P06037:12 & $\begin{array}{l}\text { Remote visual tank wall inspection } \\
\text { revealed no areas of concern since } \\
\text { last evaluated on } 7 / 28 / 05 \text {. }\end{array}$ \\
\hline $\mathrm{H}$ & 51 & $A-04 \quad(A)$ & 03/08/06 & WAP & I & P06037:09 & $\begin{array}{l}\text { Remote visual tank wall inspection } \\
\text { revealed no areas of concern since } \\
\text { last evaluated on } 7 / 28 / 05 \text {. }\end{array}$ \\
\hline $\mathrm{H}$ & 51 & $\mathrm{P}-01 \quad(\mathrm{~A})$ & 08/15/06 & $\mathrm{DP}$ & I & P06227:01-27 & $\begin{array}{l}\text { Remote visual tank wall inspection } \\
\text { revealed no areas of concern since } \\
\text { last evaluated on } 11 / 12 / 05 \text {. The } \\
\text { annulus jet beneath the F riser } \\
\text { showed no degradation per T-DS-G } \\
-00043 \text {. }\end{array}$ \\
\hline $\mathrm{H}$ & 51 & $\mathrm{P}-01 \quad(\mathrm{~A})$ & $08 / 15 / 06$ & $\mathrm{DP}$ & l & P06228:01-27 & $\begin{array}{l}\text { Remote visual inspection of the } \\
\text { secondary vessel wall revealed no } \\
\text { areas of concern since last evaluated } \\
\text { on } 11 / 27 / 05 \text {. }\end{array}$ \\
\hline $\mathrm{H}$ & 51 & $\mathrm{P}-02 \quad(\mathrm{~A})$ & 08/15/06 & DP & I & P06229:01-27 & $\begin{array}{l}\text { Remote visual tank wall inspection } \\
\text { revealed no areas of concern since } \\
\text { last evaluated on } 7 / 27 / 05 \text {. }\end{array}$ \\
\hline
\end{tabular}




\begin{tabular}{|c|c|c|c|c|c|c|c|c|}
\hline \multirow{2}{*}{ AREA } & \multirow{2}{*}{$\begin{array}{c}\text { TANK OR } \\
\text { ANCILLARY } \\
51\end{array}$} & \multicolumn{2}{|c|}{$\frac{\text { ACCESS OPENING }}{(A \text { OR I) }}$} & \multirow{2}{*}{$\frac{\text { DATE }}{03 / 08 / 06}$} & \multicolumn{3}{|c|}{$\begin{array}{l}\text { INSPECTION METHOD } \\
\text { IDENTIFICATION NUMBER }\end{array}$} & \multirow{2}{*}{$\begin{array}{l}\qquad \text { REMARKS } \\
\text { Remote visual tank wall inspection } \\
\text { revealed no areas of concern since } \\
\text { last evaluated on } 7 / 27 / 05 \text {. }\end{array}$} \\
\hline & & P-03 & (A) & & WAP & l & P06037:05 & \\
\hline $\mathrm{H}$ & 51 & P-04 & (A) & 03/08/06 & WAP & I & P06037:03 & $\begin{array}{l}\text { Remote visual tank wall inspection } \\
\text { revealed no areas of concern since } \\
\text { last evaluated on } 7 / 27 / 05 \text {. }\end{array}$ \\
\hline $\mathrm{H}$ & 51 & P-05 & (A) & 03/08/06 & WAP & I & P06037:01 & $\begin{array}{l}\text { Remote visual tank wall inspection } \\
\text { revealed no areas of concern since } \\
\text { last evaluated on } 7 / 27 / 05 \text {. }\end{array}$ \\
\hline $\mathrm{H}$ & 51 & P-06 & (A) & 03/08/06 & WAP & I & P06037:14 & $\begin{array}{l}\text { Remote visual tank wall inspection } \\
\text { revealed no areas of concern since } \\
\text { last evaluated on } 7 / 27 / 05 \text {. }\end{array}$ \\
\hline $\mathrm{H}$ & 51 & P-07 & (A) & 03/08/06 & WAP & I & P06037:13 & $\begin{array}{l}\text { Remote visual tank wall inspection } \\
\text { revealed no areas of concern since } \\
\text { last evaluated on } 7 / 27 / 05 \text {. }\end{array}$ \\
\hline $\mathrm{H}$ & 51 & P-08 & (A) & 08/15/06 & DP & I & P06230:01-27 & $\begin{array}{l}\text { Remote visual tank wall inspection } \\
\text { revealed no areas of concern since } \\
\text { last evaluated on } 7 / 27 / 05 \text {. }\end{array}$ \\
\hline $\mathrm{H}$ & 51 & P-09 & (A) & 08/15/06 & DP & l & P06231:01-27 & $\begin{array}{l}\text { Remote visual tank wall inspection } \\
\text { revealed no areas of concern since } \\
\text { last evaluated on } 7 / 27 / 05 \text {. }\end{array}$ \\
\hline $\mathrm{H}$ & 51 & $P-10$ & (A) & $03 / 08 / 06$ & WAP & I & P06037:11 & $\begin{array}{l}\text { Remote visual tank wall inspection } \\
\text { revealed no areas of concern since } \\
\text { last evaluated on } 7 / 27 / 05 \text {. }\end{array}$ \\
\hline $\mathrm{H}$ & 51 & $P-11$ & (A) & 03/08/06 & WAP & I & P06037:10 & $\begin{array}{l}\text { Remote visual tank wall inspection } \\
\text { revealed no areas of concern since } \\
\text { last evaluated on } 7 / 27 / 05 \text {. }\end{array}$ \\
\hline $\mathrm{H}$ & 51 & $\mathrm{P}-12$ & (A) & 03/08/06 & WAP & I & P06037:08 & $\begin{array}{l}\text { Remote visual tank wall inspection } \\
\text { revealed no areas of concern since } \\
\text { last evaluated on } 7 / 27 / 05 \text {. }\end{array}$ \\
\hline $\mathrm{H}$ & 51 & $P-13$ & (A) & 03/08/06 & WAP & I & P06037:07 & $\begin{array}{l}\text { Remote visual tank wall inspection } \\
\text { revealed no areas of concern since } \\
\text { last evaluated on } 7 / 27 / 05 \text {. }\end{array}$ \\
\hline $\mathrm{H}$ & 51 & $\mathrm{P}-14$ & $(\mathrm{~A})$ & 03/08/06 & WAP & l & P06037:04 & $\begin{array}{l}\text { Remote visual tank wall inspection } \\
\text { revealed no areas of concern since } \\
\text { last evaluated on } 7 / 27 / 05 \text {. }\end{array}$ \\
\hline $\mathrm{H}$ & CT & Sump & & 05/17/06 & CCTV & l & 1272 & $\begin{array}{l}\text { Inspection verified conditions of the } \\
\text { encasement walls, floor, and exterior } \\
\text { of the catch tank visible from the } \\
\text { sump riser were satisfactory per T- } \\
\text { DS-G-00049. }\end{array}$ \\
\hline
\end{tabular}




\begin{tabular}{|c|c|c|c|c|c|c|c|}
\hline \multirow{2}{*}{$\begin{array}{l}\text { AREA } \\
F\end{array}$} & \multirow{2}{*}{$\begin{array}{c}\text { TANK OR } \\
\text { ANCILLARY } \\
\\
\text { DB-02 }\end{array}$} & \multirow{2}{*}{$\frac{\frac{\text { ACCESS OPENING }}{\text { (A OR I) }}}{\text { Sump }}$} & \multirow{2}{*}{$\begin{array}{l}\text { DATE } \\
11 / 25 / 06\end{array}$} & \multicolumn{3}{|c|}{$\begin{array}{l}\text { INSPECTION METHOD } \\
\text { IDENTIFICATION NUMBER }\end{array}$} & \multirow{2}{*}{$\begin{array}{l}\text { REMARKS } \\
\text { Inspection verified conditions of the } \\
\text { walls, floor, jumpers, valves, piping } \\
\text { and cell covers visible from the sump } \\
\text { riser were satisfactory per SW11.6- } \\
\text { SVP-45, section 4.7. }\end{array}$} \\
\hline & & & & CCTV & I & 1356 & \\
\hline $\mathrm{H}$ & DB-02 & NE & 09/23/06 & CCTV & I & 1469 & $\begin{array}{l}\text { Inspection verified conditions of the } \\
\text { jumpers, piping, walls, floor, valves } \\
\text { and cell covers were satisfactory per } \\
\text { SW11.6-SVP-45 section } 4.7 \text {. }\end{array}$ \\
\hline $\mathrm{H}$ & DB-02 & SW & 08/20/06 & CCTV & I & 1141 & $\begin{array}{l}\text { Inspection verified conditions of the } \\
\text { jumpers, piping, walls, floor and } \\
\text { valves were satisfactory per SW11.6- } \\
\text { SVP-45 section } 4.7 \text {. }\end{array}$ \\
\hline$F$ & DB-03 & North & $10 / 04 / 06$ & CCTV & I & 1473 & $\begin{array}{l}\text { Inspection verified conditions of the } \\
\text { walls, floors, jumpers, valves, piping } \\
\text { and cell covers were satisfactory per } \\
\text { SW11.6-SVP-45 section } 4.7 \text {. Stains } \\
\text { and marks were observed on the } \\
\text { stainless steel liner. Additional } \\
\text { inspections will be performed to } \\
\text { determine the source of the stains. } \\
\text { Deposits on two valves indicate that } \\
\text { minor leakage has occurred at the } \\
\text { valve bodies. No other unusual } \\
\text { conditions were observed. }\end{array}$ \\
\hline$F$ & DB-03 & South & $10 / 04 / 06$ & CCTV & I & 1473 & $\begin{array}{l}\text { Inspection verified conditions of the } \\
\text { walls, floors, jumpers, valves, piping } \\
\text { and cell covers were satisfactory per } \\
\text { SW11.6-SVP- } 45 \text { section } 4.7 \text {. Stains } \\
\text { and marks were observed on the } \\
\text { stainless steel liner. Additional } \\
\text { inspections will be performed to } \\
\text { determine the source of the stains. } \\
\text { Deposits on two valves indicate that } \\
\text { minor leakage has occurred at the } \\
\text { valve bodies. No other unusual } \\
\text { conditions were observed. }\end{array}$ \\
\hline$F$ & DB-03 & South & $12 / 03 / 06$ & CCTV & I & 1473 & $\begin{array}{l}\text { Inspection was performed to } \\
\text { determine if stains observed in } \\
\text { inspection performed on } 10 / 4 / 06 \\
\text { were from water inleakage. The } \\
\text { stains observed are from rainwater } \\
\text { leaking around the cell cover and } \\
\text { water draining from Tank } 33 / 34 \text { gang } \\
\text { valve house drain line tied into the } \\
\text { vent line. This completes the } \\
\text { inspection of the pit and satisfies } \\
\text { SW11.6-SVP-45 section } 4.7 \text {. }\end{array}$ \\
\hline
\end{tabular}




\begin{tabular}{|c|c|c|c|c|c|c|}
\hline AREA & $\begin{array}{r}\text { TANK OR } \\
\text { ANCILLARY } \\
\end{array}$ & $\frac{\text { ACCESS OPENING }}{\text { (A OR I) }}$ & DATE & \multicolumn{3}{|c|}{$\begin{array}{l}\text { INSPECTION METHOD } \\
\text { IDENTIFICATION NUMBER }\end{array}$} \\
\hline$F$ & DB-04 & NW & 09/24/06 & CCTV & I & 1470 \\
\hline $\mathrm{H}$ & DB-08 & NW & 09/08/06 & CCTV & I & 1456 \\
\hline $\mathrm{H}$ & DB-08 & SW & 09/08/06 & CCTV & I & 1456 \\
\hline$F$ & EVAP-16 & E-04 & 03/16/06 & CCTV & I & 1358 \\
\hline $\mathrm{F}$ & EVAP-16 & NW & 07/10/06 & CCTV & I & 1425 \\
\hline$F$ & EVAP-16 & SE & 07/10/06 & CCTV & I & 1425 \\
\hline
\end{tabular}

Inspection verified conditions of the walls, floors, jumpers, valves, piping and cell covers were satisfactory per SW11.6-SVP-45 section 4.7 .

Inspection verified conditions of the walls, floors, jumpers, valves, piping and cell covers were satisfactory per SW11.6-SVP-45 section 4.7.

Inspection verified conditions of the walls, floors, jumpers, valves, piping and cell covers were satisfactory per SW11.6-SVP-45 section 4.7.

Inspection of the evaporator pot revealed no unusual conditions. The vessel was essentially clean and all observations were consistent with normal evaporator operation. This evaluation is documented in CBULTS-2006-00082.

Inspection verified conditions of the stainless steel liner, jumpers, valves, piping, evaporator pot exterior and cell covers were satisfactory per SW11.6-SVP-45, section 4.5 and TDS-G-00016. Stains and marks on the cell floor are from the condenser cell. An increase in mercury droplets was observed on the cell floor. The droplets are from the mercury removal tank.

Inspection verified conditions of the stainless steel liner, jumpers, valves, piping, evaporator pot exterior and cell covers were satisfactory per SW11.6-SVP-45, section 4.5 and TDS-G-00016. Stains and marks on the cell floor are from the condenser cell. An increase in mercury droplets was observed on the cell floor. The droplets are from the mercury removal tank. Additional extraneous items were observed on the cell floor. 


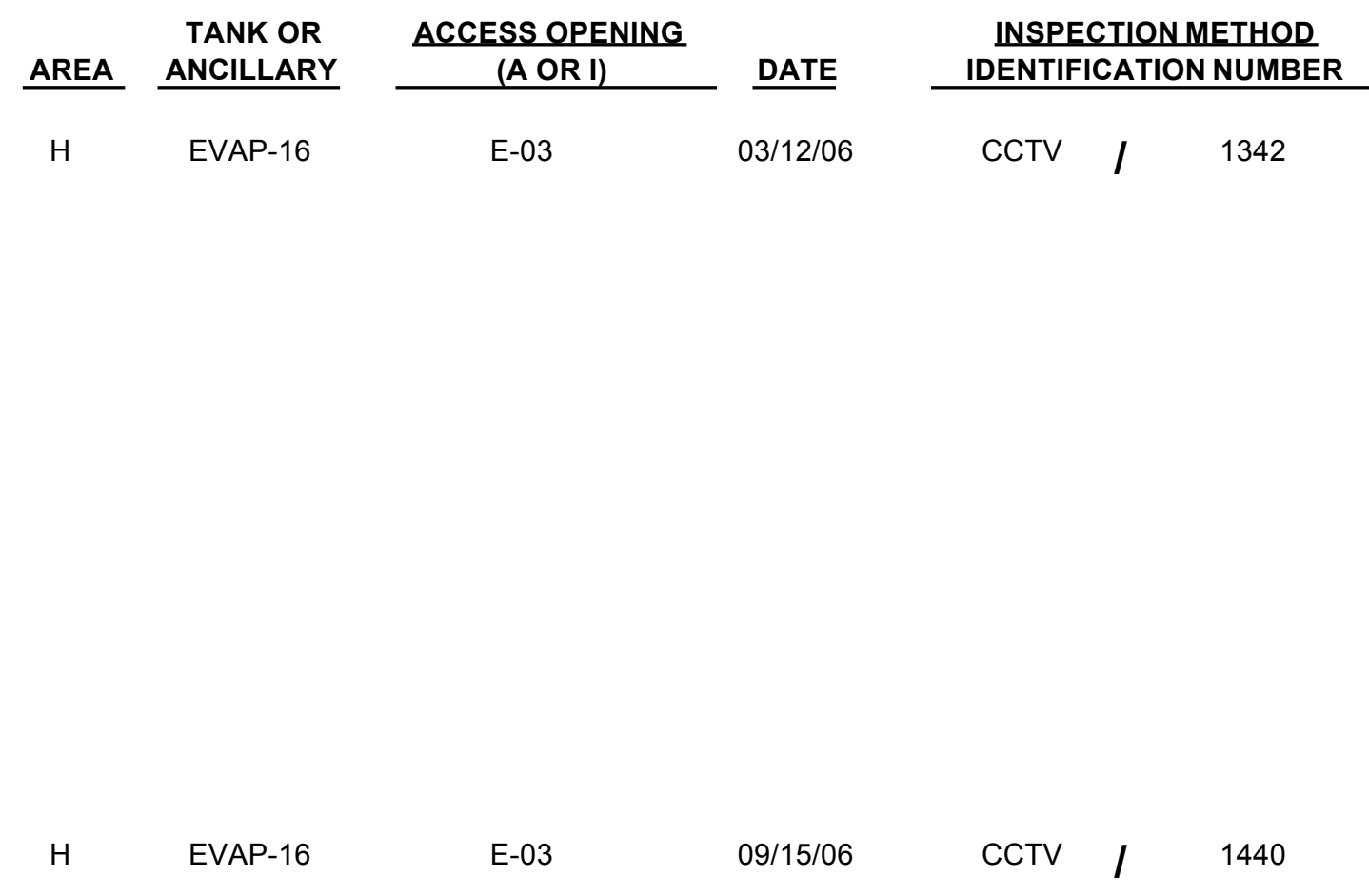

Inspection of the pot revealed the tube bundle was essentially clean with loose solids observed. The visible areas of the pot wall of the vessel were covered with scale and deposits 1/4" thick, with some nodules thicker. All of the observable piping (thermowells, feed inlet, steam lines, etc.) were coated and several deposits were observed. The material on the service piping appeared to be hardened deposits up to $1 / 2$ " thick. The bottom of the vessel was not visible through the liquid. This evaluation is documented in CBU-LTS-2006 -00073 .

Inspection of the pot revealed the tube bundle was essentially clean with loose solids observed. The visible areas of the pot wall were covered with scale and deposits 1/4" thick, with some nodules thicker. An increase in the size of accumulations of solids on one area of the wall was observed. All of the observable piping (thermowells, feed inlet, steam lines, etc.) were coated and several deposits were observed. The material on the service piping appeared to be hardened deposits up to $1 / 2$ " thick. The bottom of the vessel was not visible through the liquid. This evaluation is documented in LWO-LWE-2006 -00067 .

Inspection of the evaporator pot after chemical cleaning revealed that all solids below the operating level had been removed except for one area of the tank wall covering approximately 10.5 square feet. This area of hardened deposits had been significantly reduced in size since last evaluated on $9 / 15 / 06$. This evaluation is documented in LWOLWE-2006-00143. 


\begin{tabular}{|c|c|c|c|c|c|c|}
\hline AREA & $\begin{array}{r}\text { TANK OR } \\
\text { ANCILLARY } \\
\end{array}$ & $\frac{\text { ACCESS OPENING }}{(\text { A OR I) }}$ & DATE & \multicolumn{3}{|c|}{$\begin{array}{l}\text { INSPECTION METHOD } \\
\text { IDENTIFICATION NUMBER }\end{array}$} \\
\hline $\mathrm{H}$ & EVAP-16 & $\mathrm{NE}$ & 09/30/06 & CCTV & I & 1472 \\
\hline $\mathrm{H}$ & EVAP-16 & sW & 09/30/06 & CCTV & l & 1472 \\
\hline
\end{tabular}




\begin{tabular}{|c|c|c|c|c|c|c|c|}
\hline \multirow{2}{*}{$\frac{\text { AREA }}{\mathrm{H}}$} & \multirow{2}{*}{$\begin{array}{c}\text { TANK OR } \\
\text { ANCILLARY } \\
\begin{array}{c}\text { LDB Drain } \\
\text { Cell }\end{array}\end{array}$} & \multirow{2}{*}{$\frac{\frac{\text { ACCESS OPENING }}{\text { (A OR I) }}}{\text { Sump Riser }}$} & \multirow{2}{*}{$\begin{array}{l}\text { DATE } \\
11 / 07 / 06\end{array}$} & \multicolumn{3}{|c|}{$\begin{array}{l}\text { INSPECTION METHOD } \\
\text { IDENTIFICATION NUMBER }\end{array}$} & \multirow{2}{*}{$\begin{array}{l}\text { REMARKS } \\
\text { Inspection verified conditions of the } \\
\text { walls, floor, jumpers, valves, and } \\
\text { piping were satisfactory per SW11.6- } \\
\text { SVP-45, section 4.7. }\end{array}$} \\
\hline & & & & CCTV & I & 1503 & \\
\hline$F$ & PP-01 & SW & $10 / 14 / 06$ & CCTV & I & 1491 & $\begin{array}{l}\text { Inspection verified conditions of the } \\
\text { walls, floor, jumpers, valves, piping, } \\
\text { and cell covers visible from the sump } \\
\text { riser were satisfactory per SW11.6- } \\
\text { SVP-45, section } 4.7 \text {. The passive } \\
\text { vent was satisfactory per SW11.6- } \\
\text { SVP-45, section } 4.9 \text {. }\end{array}$ \\
\hline$F$ & PP-02 & SW & $09 / 27 / 06$ & CCTV & I & 1125 & $\begin{array}{l}\text { Inspection verified conditions of the } \\
\text { walls, floor, jumpers, valves, piping, } \\
\text { and cell covers visible from the sump } \\
\text { riser were satisfactory per SW11.6- } \\
\text { SVP-45, section } 4.7 \text {. The passive } \\
\text { vent was satisfactory per SW11.6- } \\
\text { SVP-45, section } 4.9 \text {. }\end{array}$ \\
\hline $\mathrm{H}$ & PP-02 & NE & $10 / 31 / 06$ & CCTV & I & 1447 & $\begin{array}{l}\text { Inspection verified conditions of the } \\
\text { walls, floor, jumpers, piping, and cell } \\
\text { covers visible from the northeast } \\
\text { riser were satisfactory per SW11.6- } \\
\text { SVP-45, section } 4.7 \text {. }\end{array}$ \\
\hline $\mathrm{H}$ & PP-02 & SW & $10 / 31 / 06$ & CCTV & I & 1447 & $\begin{array}{l}\text { Inspection verified conditions of the } \\
\text { walls, floor, jumpers, piping, and cell } \\
\text { covers visible from the southwest } \\
\text { riser were satisfactory per SW11.6- } \\
\text { SVP-45, section } 4.7 \text {. The passive } \\
\text { vent was satisfactory per SW11.6- } \\
\text { SVP-45, section } 4.9 \text {. }\end{array}$ \\
\hline$F$ & PP-03 & Sump & $09 / 24 / 06$ & CCTV & I & 1125 & $\begin{array}{l}\text { Inspection verified conditions of the } \\
\text { walls, floor, jumpers, valves, piping, } \\
\text { and cell covers were satisfactory per } \\
\text { SW11.6-SVP-45, section } 4.7 \text {. The } \\
\text { passive vent was satisfactory per } \\
\text { SW11.6-SVP-45, section } 4.9 \text {. }\end{array}$ \\
\hline $\mathrm{H}$ & PP-03 & NE & $10 / 05 / 06$ & CCTV & I & 1468 & $\begin{array}{l}\text { Inspection verified conditions of the } \\
\text { walls, floor, jumpers, piping, and cell } \\
\text { covers visible from the northeast } \\
\text { riser were satisfactory per SW11.6- } \\
\text { SVP-45, section } 4.7 \text {. The passive } \\
\text { vent was satisfactory per SW11.6- } \\
\text { SVP-45, section } 4.9 \text {. }\end{array}$ \\
\hline
\end{tabular}




\begin{tabular}{|c|c|c|c|c|c|c|c|}
\hline \multirow{2}{*}{$\frac{\text { AREA }}{\mathrm{H}}$} & \multirow{2}{*}{$\begin{array}{c}\begin{array}{c}\text { TANK OR } \\
\text { ANCILLARY }\end{array} \\
\text { PP-03 }\end{array}$} & \multirow{2}{*}{$\begin{array}{c}\frac{\text { ACCESS OPENING }}{\text { (A OR I) }} \\
\text { SW }\end{array}$} & \multirow{2}{*}{$\begin{array}{c}\text { DATE } \\
10 / 31 / 06\end{array}$} & \multicolumn{3}{|c|}{$\begin{array}{l}\text { INSPECTION METHOD } \\
\text { IDENTIFICATION NUMBER }\end{array}$} & \multirow{2}{*}{$\begin{array}{l}\text { REMARKS } \\
\text { Inspection verified conditions of the } \\
\text { walls, floor, jumpers, piping, and cell } \\
\text { covers visible from the southwest } \\
\text { riser were satisfactory per SW11.6- } \\
\text { SVP-45, section 4.7. Also, the cover } \\
\text { plate on top of the pump tank is not } \\
\text { properly seated. }\end{array}$} \\
\hline & & & & CCTV & I & 1447 & \\
\hline $\mathrm{H}$ & PP-04 & NE & $09 / 23 / 06$ & CCTV & I & 1468 & $\begin{array}{l}\text { Inspection verified conditions of the } \\
\text { walls, floor, jumpers, piping, and cell } \\
\text { covers visible from the northeast } \\
\text { riser were satisfactory per SW11.6- } \\
\text { SVP-45, section } 4.7 \text {. The passive } \\
\text { vent was satisfactory per SW11.6- } \\
\text { SVP-45, section } 4.9 \text {. }\end{array}$ \\
\hline $\mathrm{H}$ & PP-04 & SW & 09/23/06 & CCTV & I & 1468 & $\begin{array}{l}\text { Inspection verified conditions of the } \\
\text { walls, floor, jumpers, piping, and cell } \\
\text { covers visible from the southwest } \\
\text { riser were satisfactory per SW11.6- } \\
\text { SVP-45, section } 4.7 \text {. The passive } \\
\text { vent was satisfactory per SW11.6- } \\
\text { SVP-45, section } 4.9 \text {. }\end{array}$ \\
\hline $\mathrm{H}$ & PP-07 & NW & $06 / 17 / 06$ & CCTV & I & 1409 & $\begin{array}{l}\text { Inspection verified conditions of the } \\
\text { walls, cell covers, jumpers, valves, } \\
\text { pump tank exterior and floor were } \\
\text { satisfactory per SW11.6-SVP-45, } \\
\text { section } 4.7 \text {. The passive vent was } \\
\text { satisfactory per SW11.6-SVP-45, } \\
\text { section } 4.9 \text {. }\end{array}$ \\
\hline $\mathrm{H}$ & PP-07 & SE & $06 / 17 / 06$ & CCTV & I & 1409 & $\begin{array}{l}\text { Inspection verified conditions of the } \\
\text { walls, cell covers, jumpers, valves, } \\
\text { pump tank exterior and floor were } \\
\text { satisfactory per SW } 11.6-S V P-45 \text {, } \\
\text { section } 4.7 \text {. The passive vent was } \\
\text { satisfactory per SW11.6-SVP-45, } \\
\text { section } 4.9 \text {. }\end{array}$ \\
\hline $\mathrm{H}$ & PP-08 & NW & 06/17/06 & CCTV & I & 1408 & $\begin{array}{l}\text { Inspection verified conditions of the } \\
\text { walls, cell covers, jumpers, valves, } \\
\text { pump tank exterior and floor were } \\
\text { satisfactory per SW } 11.6-\text { SVP- } 45 \text {, } \\
\text { section } 4.7 \text {. The passive vent was } \\
\text { satisfactory per SW } 11.6-S V P-45 \text {, } \\
\text { section } 4.9 \text {. }\end{array}$ \\
\hline
\end{tabular}




\begin{tabular}{|c|c|c|c|c|c|c|c|}
\hline \multirow{2}{*}{$\frac{\text { AREA }}{\mathrm{H}}$} & \multirow{2}{*}{ 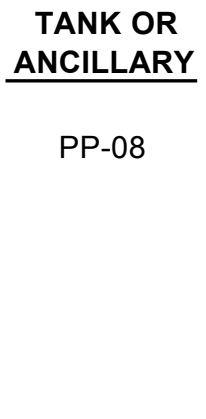 } & \multirow{2}{*}{$\begin{array}{c}\frac{\text { ACCESS OPENING }}{\text { (A OR I) }} \\
\text { SE }\end{array}$} & \multirow{2}{*}{$\begin{array}{c}\text { DATE } \\
06 / 17 / 06\end{array}$} & \multicolumn{3}{|c|}{$\begin{array}{l}\text { INSPECTION METHOD } \\
\text { IDENTIFICATION NUMBER }\end{array}$} & \multirow{2}{*}{$\begin{array}{l}\text { REMARKS } \\
\text { Inspection verified conditions of the } \\
\text { walls, cell covers, jumpers, valves, } \\
\text { pump tank exterior and floor were } \\
\text { satisfactory per SW11.6-SVP-45, } \\
\text { section 4.7. The passive vent was } \\
\text { satisfactory per SW11.6-SVP-45, } \\
\text { section 4.9. }\end{array}$} \\
\hline & & & & CCTV & I & 1408 & \\
\hline $\mathrm{H}$ & PP-09 & NW & $06 / 17 / 06$ & CCTV & I & 1407 & $\begin{array}{l}\text { Inspection verified conditions of the } \\
\text { walls, cell covers, jumpers, valves, } \\
\text { pump tank exterior and floor were } \\
\text { satisfactory per SW 11.6-SVP-45, } \\
\text { section 4.7. The passive vent was } \\
\text { satisfactory per SW 11.6-SVP-45, } \\
\text { section 4.9. }\end{array}$ \\
\hline $\mathrm{H}$ & PP-09 & SE & $06 / 17 / 06$ & CCTV & I & 1407 & $\begin{array}{l}\text { Inspection verified conditions of the } \\
\text { walls, cell covers, jumpers, valves, } \\
\text { pump tank exterior and floor were } \\
\text { satisfactory per SW11.6-SVP-45, } \\
\text { section 4.7. The passive vent was } \\
\text { satisfactory per SW11.6-SVP-45, } \\
\text { section 4.9. }\end{array}$ \\
\hline $\mathrm{H}$ & PP-10 & NW & 06/17/06 & CCTV & I & 1410 & $\begin{array}{l}\text { Inspection verified conditions of the } \\
\text { walls, cell covers, jumpers, valves, } \\
\text { pump tank exterior and floor were } \\
\text { satisfactory per SW } 11.6-\text {-SVP-45, } \\
\text { section 4.7. The passive vent was } \\
\text { satisfactory per SW 11.6-SVP-45, } \\
\text { section 4.9. }\end{array}$ \\
\hline $\mathrm{H}$ & PP-10 & SW & $06 / 17 / 06$ & CCTV & I & 1410 & $\begin{array}{l}\text { Inspection verified conditions of the } \\
\text { walls, cell covers, jumpers, valves, } \\
\text { pump tank exterior and floor were } \\
\text { satisfactory per SW } 11.6-S V P-45 \text {, } \\
\text { section } 4.7 \text {. The passive vent was } \\
\text { satisfactory per SW } 11.6-S V P-45 \text {, } \\
\text { section } 4.9 \text {. }\end{array}$ \\
\hline
\end{tabular}


WSRC-TR-2006-00170

This page intentionally left blank. 



\section{DISTRIBUTION}

\section{DOE-SR}

M. A. Mikolanis, 707-H

M. P. Dholakia (4), 707-H

D. J. Blake, 707-H

L. T. Ling, 704-S

\section{WSRC}

W. G. Poulson, 766-H

L. D. Olson, 766-H

W. D. Stephens, 703-H

R. L. Salizzoni, 703-H

G. D. Arthur, 703-H

D. J. Martin, 703-H

R. M. Campbell, 742-A

N. R. Davis, 766-H

N. F. Chapman, 703-H

M. N. Borders, 704-26F

W. C. Clark, 704-56H

D. H. Coleman, 704-26F

R. V. Doniphan, 241-156H

M. D. Buxton, 241-156H

E. Patten, 704-26F
K. A. Hauer, 766-H

N. C. Iyer (5), 773-41A

E. J. Freed, 704-56H

C. L. Atseff, 703-H

W. L. Payne, 735-B

J. W. Cook, 730-4B

J. J. Phillips, 703-H

J. B. Elder, 730-A

W. R. West (15), 704-8H

R. S. Waltz (15), 704-8H

R. D. Robbins, 704-8H

D. C. Blair, $704-8 \mathrm{H}$

M. T. Jackson, 704-8H

A. S. Plummer, 703-H

P. D. Hunt, 704-56H 